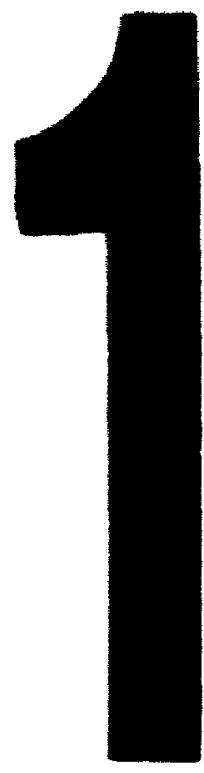

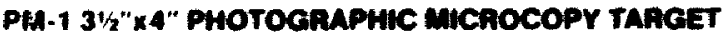
NBS 1010 a AMsiliso H2 EOUNALENT

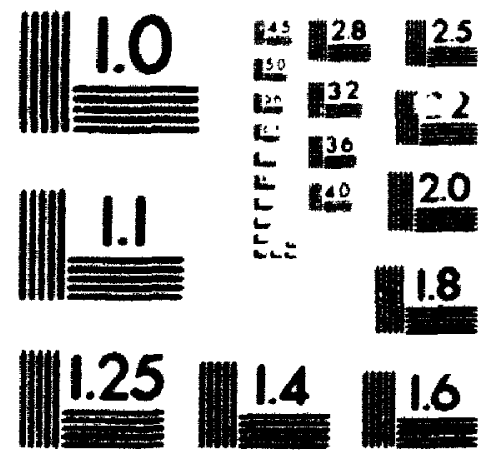


Acquisitions and

Bibliographic Services Branch

395 Wellington Street

Onawa. Ontano

KiA ON4
Biblıthèque natıonale

du Canada

Direction des acquisitions et des services bibliographiques

395. rue Wellington

Onawa (Ontaro)

KIA ON4
NOTICE

The quality of this microform is heavily dependent upon the quality of the original thesis submitted for microfilming. Every effort has been made to ensure the highest quality of reproduction possible.

If pages are missing, contact the university which granted the degree.

Some pages may have indistinct print especially if the original pages were typed with a poor typewriter ribbon or if the university sent us an inferior photocopy.

Reproduction in full or in part of this microform is governed by the Canadian Copyright Act, R.S.C. 1970, C. C-30, and subsequent amendments.
La qualité de cette microforme dépend grandement de la qualité de la thése socimise au microfilmage. Nous avons tout fait pour assurer une qualité supérieure de reproduction.

S'il manque des pages, veuillez communiquer avec l'université qui a conféré le grade.

La qualité d'impression de certaines pages peut laisser à désirer, surtout si les pages originales ont été dactylographiées à l'aide d'un ruban usé ou si l'université nous a fait parvenir une photocopie de qualité inférieure.

La reproduction, même partielle, de cette microforme est soumise à If Loi canadienne sur le droit d'auteur, SRC 1970, c. C-30, et ses amendements subséquents. 


\title{
CONTROLLED OSCILLATION OF FOREBODY VORTICES BY NOZZLE JET BLOWING
}

\author{
by \\ Karim Alexan \\ B. Eng. (Mechanical)
A thesis submitted to
the Faculty of Graduate Studies and Research
in partial fulfillment of
the requirements for the degree of \\ Master of Engineering \\ Department of Mechanical and Aerospace Engineering \\ The Ottawa-Carleton Institute for \\ Mechanical and Aerospace Engineering \\ Carleton University \\ Ottawa, Ontario \\ December 1992 \\ - copyright \\ 1992, Karim Alexan
}


Acquisitions and

Bibliographic Sevices Branch

395 Wellington Street

Ontawa Ontano

K1A ON4 du Canada

Direction des acquisitions el

des services bibliographiques

395. ne Welingtor!

Ottawa (Ontano)

KIAONA
The author has granted an irrevocable non-exclusive licence allowing the National Library of Canada to reproduco, loan, distribute or sell copies of his/her thesis by any means and in any form or format. making this thesis available to interested persons.
L'auteur a accordé une licence irrévocable ot non exclusive permettant à la Bibliothèque nationale du Canada de reproduire, prêter, distribuer ou vendre des copies de sa thèse de quelque manière et sous quelque forme que ce soit pour mettre des exemplaires de cette thèse à la disposition des personnes intéressées.

L'auteur conserve la propriété du droit d'auteur qui protège sa thèse. Ni la thèse ni des extraits substantiels de celle-ci ne doivent étre imprimés ou autrement reproduits sans son autorisation. 


\section{Carleton University Ottawa, Canada K1S5J7}

Thesis contains coloured photographe 8 /or explanatory tables which when microfilmed may lose their significance. The hardcopy of the thesig is available upon request from Carleton University Library. 


\section{Acceptance Sheet}

The undersigned recommend to the Faculty of Graduate Studies and Research acceptance of the thesis

Controlled Oscillation of Forebody Vortices

by Nozzle Jet Blowing

submitted by Karim Alexan, B. Eng.

in partial fulfillment of the requirements for

the degree of Master of Engineering.

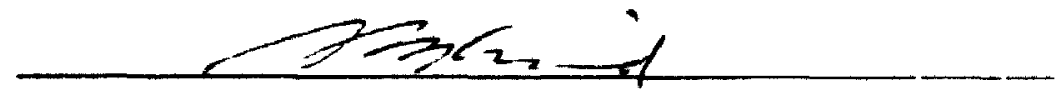

Thesis Supervisor

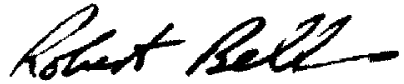

Chair, The Department of

Mechanical and Aerospace Engineering

Carleton University

January 1993 


\begin{abstract}
The flowfield surrounding an ogive-cylinder-type model immersed in a free stream fluid as its angle of attack is increased is discussed and the most promising methods of forebody vortex manipulation are reviewed. A novel scheme for controlling the behaviour of forebody vortices at high angles of attack is experimentally investigated in a water tunnel at low speed. A detailed log of the repair work and modifications done to the water tunnel, a summary of operating procedures, and recommendations for its continued successful operation are provided. The use of port and starboard positioned, forward blowing, nozzles located near the forebody apex is investigated. The ability of blowing to alter the orientation of asymmetrically positioned streamwise vortices is assessed and the possibility of using relatively rapid port-starboard oscillatory blowing to obtain a desired time-averaged side force and yaw moment, either zero or otherwise, is explored. The dynamic response of an F-18 fighter aircraft to rapid oscillatory blowing at high angles of attack is approximated. The resulting amplitudes of oscillation indicate that this motion would likely be felt by the pilot but suggestions are made for improved performance of this oscillatory blowing system. Some recommendations for future research into oscillatory blowing for forebody vortex manipulation are made.
\end{abstract}




\section{Acknowledgements}

The author wishes to thank the following:

- Mr. S. Szick and Mr. P. Trudel of the Engineering Technology Centre for their contributions to the manufacture of the test model assembly and water tunnel components.

- The Science Technology Centre for the precision machining of the forebodies, accurate positioning of the injection nozzles, and for the use of their shadowgraph.

- Mr. R. Ellis for strain gauge installation and subsequent troubleshooting assistance.

- Mr. F. Barrett for the design and assembly of the frequency control unit.

- Mr. L. Kimmel for always having a supplier in mind, a purchase order at hand, and for every little tool required by the author being made available at any time. without hesitation.

- And, a very special thank-you to Dr. R.J. Kind and Dr. E.S. Hanff for their expert knowledge, guidance and generosity. 


\section{Table of Contents}

Acceptance Sheet $\ldots \ldots \ldots \ldots \ldots \ldots \ldots \ldots \ldots \ldots \ldots \ldots \ldots \ldots$

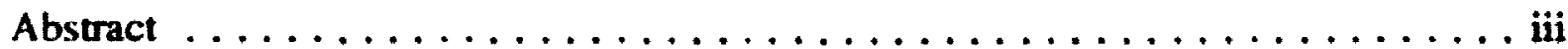

Acknowledgements $\ldots \ldots \ldots \ldots \ldots \ldots \ldots \ldots \ldots \ldots \ldots \ldots \ldots$ iv

Table of Contents $\ldots \ldots \ldots \ldots \ldots \ldots \ldots \ldots \ldots \ldots \ldots$

List of Figures $\ldots \ldots \ldots \ldots \ldots \ldots \ldots \ldots \ldots \ldots \ldots \ldots \ldots$

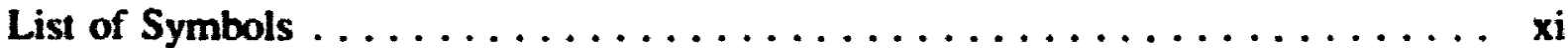

English Symbols $\ldots \ldots \ldots \ldots \ldots \ldots \ldots \ldots \ldots \ldots \ldots \ldots \ldots$

Greek Symbols . . . . . . . . . . . . . . . . . . xiii

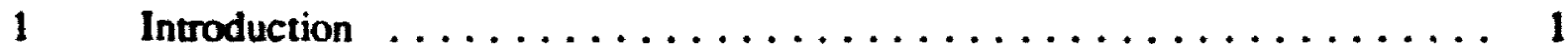

$2 \quad$ Review of Previous Work . . . . . . . . . . . . . . . 3

2.1 Rotatable Elliptical Tip $\ldots \ldots \ldots \ldots \ldots \ldots \ldots$

2.2 Strakes ............................ 9

2.3 Miniature Rotatable Nose Tip Strakes . . . . . . . . . . . 13

2.4 Blowing Jets . . . . . . . . . . . . . . . . . . 15

2.4 .1 Blowing Normal $\ldots \ldots \ldots \ldots \ldots \ldots$

2.4.2 Blowing Tangentially Forward ............... 17

2.4.3 Blowing Tangentially Aft .................. 19

2.4.4 Effects of Nozzle Exit Geometry .............. 21

2.4.5 Pulsed Blowing Jets ................... 22

2.4.6 Aft and Forward Blowing Combined . . . . . . . . . . 23

2.5 Strakes and Tangential Blowing Combined ............. 23

2.6 Blowing Slots ........................ 26

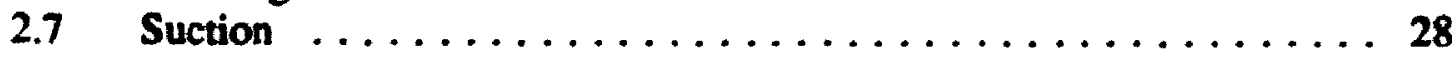

2.8 Overview of Control Methods . . . . . . . . . . . . . . . . . . . 29

2.9 Transient Behaviour of the Lateral Loads . . . . . . . . . . . . . . . . 30

2.10 Proposed Method to Alleviate Lateral Loading . . . . . . . . . . . 31

3 Description of Apparatus and Experiments ............. 32

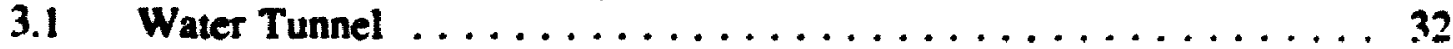

3.2 Test Equipment . . . . . . . . . . . . . . . . . 39

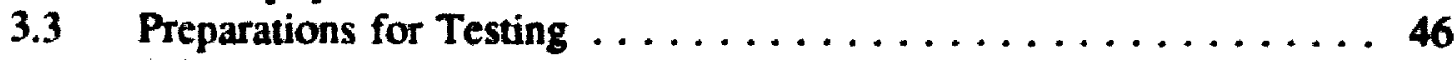

3.3 .1 Model Alignment ................... 46

3.3 .2 Troubleshooting $\ldots \ldots \ldots \ldots \ldots \ldots$

3.3 .3 Calibration ........................ 49 
3.3.4 Nondimensionalising $\ldots \ldots \ldots \ldots \ldots \ldots \ldots \ldots \ldots \ldots .50$

3.4 Experimental Procedures $\ldots \ldots \ldots \ldots \ldots \ldots \ldots \ldots \ldots \ldots$ 51

$4 \quad$ Results and Discussions $\ldots \ldots \ldots \ldots \ldots \ldots \ldots \ldots \ldots$

$4.1 \quad$ Adopted Sign Convention $\ldots \ldots \ldots \ldots \ldots \ldots \ldots \ldots \ldots \ldots$

4.2 Clean-Forebody Flowfield $\ldots \ldots \ldots \ldots \ldots \ldots \ldots \ldots \ldots$

4.3 Preliminary Nozzle Configuration $\ldots \ldots \ldots \ldots \ldots \ldots \ldots \ldots 57$

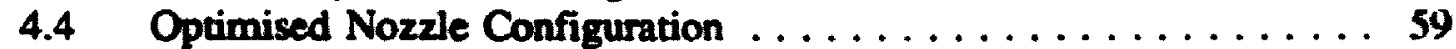

4.4.1 The Baseline Dowfield . . . . . . . . . . . . . 59

4.4.2 Baseline Flow Visualisation Test Kesults ......... 59

4.4.3 Baseline Flowfield Sensitivity to Reynolds Number . . . . 63

4.5 Blowing Effects on the Baseline Flow field . . . . . . . . . 63

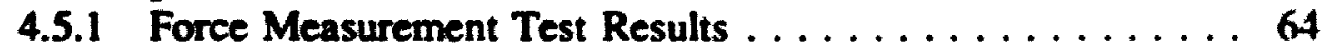

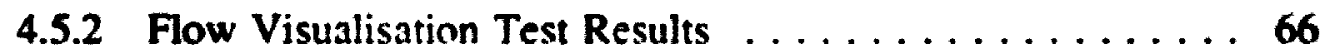

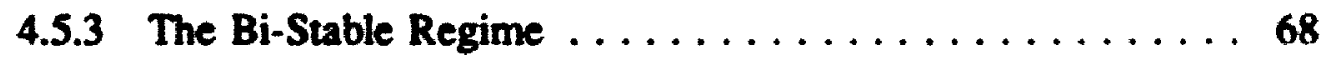

4.5.4 Individual Port and Starboard Blowing $\ldots \ldots \ldots \ldots \ldots 9$

4.6 Maximum Allowable Frequency of Oscillatory Blowing $\ldots \ldots, 70$

4.7 Oscillatory Blowing Effects $\ldots \ldots \ldots \ldots \ldots \ldots \ldots \ldots \ldots, \ldots \ldots$

4.8 Increased Frequency Testing $\ldots \ldots \ldots \ldots \ldots \ldots \ldots \ldots \ldots$

4.9 Aircraft Inertial Response $\ldots \ldots \ldots \ldots \ldots \ldots \ldots \ldots \ldots . \ldots 5$

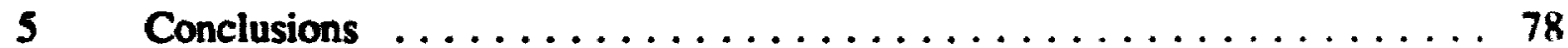

$5.1 \quad$ Water Tunnel $\ldots \ldots \ldots \ldots \ldots \ldots \ldots \ldots \ldots \ldots \ldots 78$

5.2 Experiments with the Clean-Forebody $\ldots \ldots \ldots \ldots \ldots \ldots \ldots 78$

5.3 Experiments with Nozzles $\ldots \ldots \ldots \ldots \ldots \ldots \ldots \ldots \ldots .79$

$6 \quad$ Recommendations for Future Work $\ldots \ldots \ldots \ldots \ldots \ldots$

6.1 Water Tunnel $\ldots \ldots \ldots \ldots \ldots \ldots \ldots \ldots \ldots \ldots \ldots$

6.2 Test Equipment $\ldots \ldots \ldots \ldots \ldots \ldots \ldots \ldots \ldots \ldots \ldots \ldots$

6.3 Experiments $\ldots \ldots \ldots \ldots \ldots \ldots \ldots \ldots \ldots \ldots \ldots \ldots$

References $\ldots \ldots \ldots \ldots \ldots \ldots \ldots \ldots \ldots \ldots \ldots \ldots \ldots \ldots \ldots \ldots$

Figures $\ldots \ldots \ldots \ldots \ldots \ldots \ldots \ldots \ldots \ldots \ldots \ldots \ldots \ldots$

Appendix A: Equipment Lists and Specifications $\ldots \ldots \ldots \ldots \ldots \ldots$

Appendix B: Sting and Balance Characteristics $\ldots \ldots \ldots \ldots \ldots \ldots \ldots$

Appendix C: Side Force and Yaw Moment Equations $\ldots \ldots \ldots \ldots 139$

Appendix D: Analysis of Lateral Oscillations for the F-18 Aircraft $\ldots \ldots \ldots 142$

Appendix E: Water Tunne! Operating Procedures $\ldots \ldots \ldots \ldots \ldots \ldots \ldots 147$ 


\section{List of Figures}

Figure 2.1 Flowfield description around an ogive-type forebody-cylinder model for increasing angle of attack. . . . . . . . . . . . . 89

Figure 2.2 (a) Schematic of generic fighter model. . . . . . . . . . . . . . 89

Figure 2.2(b) Yaw moment coefficient for generic fighter model. . . . . . . . . 90

Figure 2.2(c) Fc: sbody contribution to yaw moment coefficient of generic fighter model. . . . . . . . . . . . . . . . . . . . . 90

Figure 2.3 Yaw moment coefficient for various nudder deflections of generic fighter model. . . . . . . . . . . . . . . . . 91

Figure 2.4 Rotatable elliptical tip schematic. . . . . . . . . . . . . . . . 91

Figure 2.5(a) Side force coefficient versus tip roll position for the sharp axisymmetric tip. . . . . . . . . . . . . . . 92

Figure 2.5(b) Side force versus tip roll position for the blunt axisymmetric tip. . 92

Figure 2.6(a) Side force versus tip roll position for elliptical tip $01 . \ldots \ldots \ldots 93$

Figure 2.6(b) Side force versus tip roll position for elliptical tip $02 . \ldots \ldots$

Figure 2.7 Fluid mechanics of various forebody vortex control methods. . . . . 94

Figure 2.8 Variation of yaw moment with strake radial position, $\delta_{2}=0^{\circ}$, $\alpha=50^{\circ} \ldots \ldots \ldots \ldots \ldots \ldots \ldots \ldots \ldots \ldots \ldots \ldots \ldots . \ldots \ldots \ldots$

Figure 2.9 Pressure coefficients and crossflow schematics for a single strake at $\phi_{1}=60$ and $90^{\circ}, \delta_{1}=0^{\circ}, \alpha=50^{\circ} . \ldots \ldots \ldots \ldots \ldots \ldots \ldots \ldots \ldots \ldots \ldots$

Figure 2.10(a) Yaw moment coefficients for strakes positioned symmetrically at $\phi_{1}= \pm 150^{\circ}$.

Figure 2.10(b) Yaw moment coefficients for strakes positioned symmetrically at

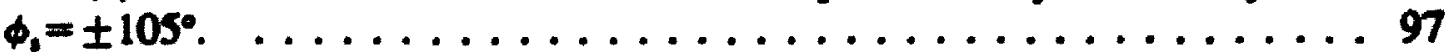

Figure 2.11 Schematic of rotatable nose tip strakes. . . . . . . . . . . . . . 98

Figure 2.12 Yaw moment versus angle of attack for forward blowing with nozzles at 2 different longitudinal stations. . . . . . . . . . . 98

Figure 2.13 Various nozzle exit geometries tested by Gittner and Chokani (1992). $\bar{y} / d$ is the nozzle nondimensional geometric mean height, $z_{\text {max }} / d$ is the nondimensional width. . . . . . . . . . . . . . . . . .999

Figure 2.14(a) Various nozzle exit geometries tested by Cornelius et al (1992). . 100

Figure 2.14(b) Schematic defining exit nozzle orientation. . . . . . . . . 100

Figure 2.15 Yaw moment versus angle of attack for combined forward and aft blowing with symmetric strakes. . . . . . . . . . . . . . . . . . 101

Figure 2.16 Yaw moment versus angle of attack for symmetric strakes with aft tangential blowing. . . . . . . . . . . . . . . . . . 101

Figure 2.17 Comparison of flowfield schematics for various nozzle jet blowing configurations. . . . . . . . . . . . . . . . . . . . 102

Figure 2.18(a) Transient behaviour of the yaw moment coefficient for an aft blowing jet, $\alpha=50^{\circ}$. . . . . . . . . . . . . . . . . 102

Figure 2.18(b) Transient behaviour of the yaw moment coefficient for a miniature rotatable nose tip strake rotation, $\alpha=50^{\circ}$. 
Figure 3.1 Carleton University water tunnel test facility. . . . . . . . . . 103

Figure 3.2 Top view of test section showing model-support insert. . . . . . . 104

Figure 3.3 Low speed drive motor, gearbox and clutch. . . . . . . . . . . . . 104

Figure 3.4 Forebody manufacturing drawing. Inset: Nozzles positioned at $\mathrm{x} / \mathrm{d}=0.25, \phi_{\mathrm{j}}= \pm 135^{\circ}$ shown flush-mounted. Note: radial position of nozzles is not accurately represented in this cross section. . . . . . . 105

Figure 3.5 Various nosecones used in experiments. . . . . . . . . . . . 106

Figure 3.6 Model assembly. . . . . . . . . . . . . . . . . 106

Figure 3.7 Schematic of injection plumbing configuration. . . . . . . . . 107

Figure 3.8(a) Components of experimental setup. . . . . . . . . . . . . . 107

Figure 3.8(b) Components of experimental setup continued. . . . . . . . . . . 108

Figure 3.9 Square wave signal initially picked up by the stripchart recorder before appropriate shielding was installed. Sensitivity: $0.04 \mathrm{~V} / \mathrm{cm}$, chart speed: $2.5 \mathrm{~cm} / \mathrm{min} . \ldots \ldots \ldots \ldots \ldots \ldots \ldots \ldots \ldots$

Figure 3.10 Schematic of strain gauge wiring configuration. . . . . . . . . 109

Figure 3.11 (a) Waterproofing of strain gauges. . . . . . . . . . . . 109

Figure $3.11($ b) Waterproofing of strain gauge leadwires. . . . . . . . . 110

Figure 3.12 Free stream velocity calibration curve. . . . . . . . . . 110

Figure 3.13 Voltage output versus applied calibration force for forward and aft mounted strain gauge bridge stations. . . . . . . . . . . . . 111

Figure 4.1 Schematic of adopted sign convention, plan view. . . . . . . . . 111

Figure 4.2(a) Side force versus angle of attack for the clean-forebody. $\beta=0^{\prime \prime}$,

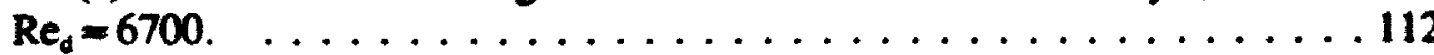

Figure 4.2(b) Yaw moment versus angle of attack for the clean-forebody. $\beta=0^{\circ}$, $\operatorname{Re}_{\mathrm{d}} \mathbf{2} 6700 . \ldots \ldots \ldots \ldots \ldots \ldots \ldots \ldots \ldots \ldots \ldots \ldots \ldots$

Figure 4.2(c) Forward strain gauge bridge station stripchart output for the cleanforebody flowfield. $\beta=0^{\circ}, \operatorname{Re}_{\mathrm{d}} \approx 6700$, sensitivity: $0.04 \mathrm{~V} / \mathrm{cm}$, chart speed:

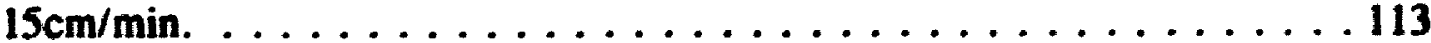

Figure 4.3(a) Mean side force versus angle of attack for the clean-forebody. $\mathrm{Re}_{\mathrm{d}} \approx 6700$.

Figure 4.3(b) Mean yaw moment versus angle of attack for the clean-forebody. $\mathrm{Re}_{\mathrm{d}} \approx \mathbf{6 7 0 0} . \quad \ldots \ldots \ldots \ldots \ldots \ldots \ldots \ldots \ldots \ldots \ldots \ldots$

Figure 4.4(a) Mean side force versus angle of attack for the baseline flowfield.

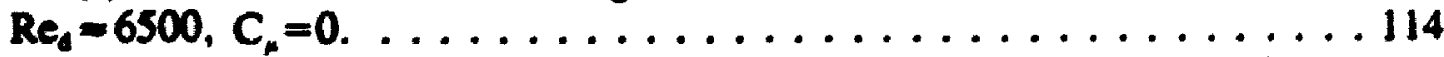

Figure 4.4(b) Mean yaw moment versus angle of attack for the baseline

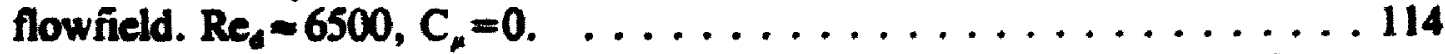

Figure 4.5(a) Side force versus angle of attack for the baseline flowfield. $\beta=0^{\circ}$,

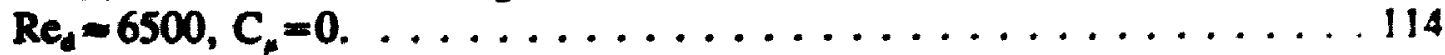

Figure 4.5(b) Yaw moment versus angle of attack for the baseline flowfield. $\beta=0^{\circ}, \operatorname{Re}_{\mathrm{d}}=6500, C_{\mathrm{p}}=0 . \ldots \ldots \ldots \ldots \ldots \ldots \ldots \ldots \ldots \ldots \ldots \ldots$

Figure 4.6 Flow visualisation of the baseline flowfield. $\alpha=50^{\circ}, \beta=0^{\circ}$, $R e_{\mathrm{d}}=1900, \mathrm{C}_{\mathrm{r}}=0 . . \ldots \ldots \ldots \ldots \ldots \ldots \ldots \ldots \ldots \ldots \ldots \ldots \ldots$

Figure 4.7(a) Baseline flowfield sensitivity to Reynolds number. Side force versus angle of attack. $\beta=0^{\circ}, C_{\mu}=0$. 
Figure 4.7(b) Baseline flowfield sensitivity to Reynolds number. Yaw moment versus angle of attack. $\beta=0, C_{\mu}=0 . \ldots \ldots \ldots \ldots \ldots \ldots \ldots$

Figure 4.8 Stabilising effect of blowing. $\alpha=50^{\circ}, \beta=0^{\circ}, R e_{d}=7200$, $C_{n}= \pm 0.0013, \bar{\omega}=0$, sensitivity: $0.04 \mathrm{~V} / \mathrm{cm}$, chart speed: $2.5 \mathrm{~cm} / \mathrm{min} . \ldots 117$

Figure 4.9 Control and stabilising effects of blowing. $\alpha=60^{\circ}, \beta=0^{\circ}, R_{e}=7200$, $C_{n}= \pm 0.0013, \bar{\omega}=0$, sensitivity: $0.04 \mathrm{~V} / \mathrm{cm}$, chart speed: $2.5 \mathrm{~cm} / \mathrm{min} . \ldots 118$

Figure 4.10(a) Mean side force versus coefficient of momentum blowing for the optimised configuration. $\alpha=50^{\circ}, \beta=0^{\circ}, \mathrm{Re}_{\mathrm{e}}=6900, \bar{\omega}=0$.

Figure 4.10(b) Mean yaw moment versus coefficient of momentum blowing for the optimised configuration. $\alpha=50^{\circ}, \beta=0^{\circ}, R_{e}=6900, \bar{\omega}=0$.

Figure 4.10(c) Steady-state mean yaw moment as a function of blowing for a $6 \%$ scale F/A-18 fighter aircraft model.

Figure 4.11 Flow visualisation of starboard blowing. $\alpha=50^{\circ}, \beta=0^{\circ}, R e_{d}=1900$, $C_{4}=+0.0013, \bar{\omega}=0$.

Figure 4.12 Flow visualisation of port blowing. $\alpha=50^{\circ}, \beta=0^{\circ}, R e_{d}=1900$,

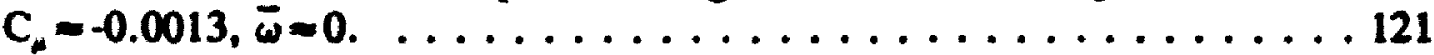

Figure 4.13 Flow visualisation of starboard blowing. $\alpha=70^{\circ}, \beta=0^{\circ}, R e_{d}=1900$, $C_{-}=+0.0013, \bar{\omega}=0$.

Figure 4.14 Forebody vortex control in the bi-stable flow regime. $\alpha=50^{\circ}, \beta=0^{\circ}$, $\mathrm{Re}_{\mathrm{d}}=7200, \mathrm{C}_{\alpha}= \pm 0.0013, \bar{\omega}=0$, sensitivity: $0.04 \mathrm{~V} / \mathrm{cm}$, chart speed: $2.5 \mathrm{~cm} / \mathrm{min}$.

Figure 4.15(a) Individual port and starboard blowing. $\beta=0{ }^{\circ}, R_{d}=7200$,

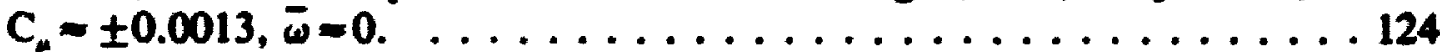

Figure 4.15(b) Individual port and starboard blowing. $\beta=-10^{\circ}, \operatorname{Re}_{\mathrm{d}}=7200$, $C_{n}= \pm 0.0013, \bar{\omega}=0$.

Figure 4.15(c) Individual port and starboard blowing. $\beta=-20^{\circ}, R e_{d}=7200$, $C_{n}= \pm 0.0013, \bar{\omega}=0$.

Figure 4.16 Estimation of maximum allowable frequency of oscillatory blowing. $\alpha=50^{\circ}, \beta=0^{\circ}, R e_{d}=7200, C_{\mu}= \pm 0.0013$, sensitivity: $0.04 \mathrm{~V} / \mathrm{cm}$, chart

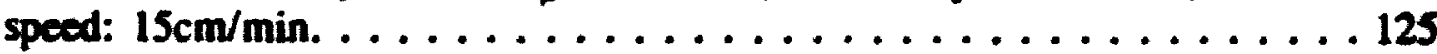

Figure 4.17(a) Typical stripchart output for oscillatory blowing below the maximum allowable frequency. $\alpha=50^{\circ}, \beta=0^{\circ}, \mathrm{Re}_{d}=7100, \mathrm{C}_{n}= \pm 0.0013$, $\bar{\omega}=0.08$, sensitivity: $0.04 \mathrm{~V} / \mathrm{cm}$, chart speed: $15 \mathrm{~cm} / \mathrm{min}$.

Figure 4.17(b) Typical stripchart output for oscillatory blowing at the maximum allowable frequency. $\alpha=50^{\circ}, \beta=0^{\circ}, \operatorname{Re}_{d}=7400, C_{\mu}= \pm 0.0012, \bar{\omega}=0.16$, sensitivity: $0.04 \mathrm{~V} / \mathrm{cm}$, chart speed: $15 \mathrm{~cm} / \mathrm{min} . \ldots \ldots \ldots \ldots \ldots$

Figure 4.18(a) Port and starboard oscillatory blowing. $\beta=0{ }^{\circ}, R e_{d}=7100$,

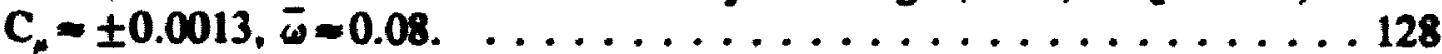

Figure 4.18(b) Port and starboard oscillatory blowing. $\beta=-10^{\circ}, R_{d}=7100$, $C_{\mu}= \pm 0.0013, \bar{\omega}=0.08$.

Figure 4.18(c) Port and starboard oscillatory blowing. $\beta=-20^{\circ}, R e_{d}=7100$, $C_{.}= \pm 0.0013, \bar{\omega}=0.08$.

Figure $4.19(2)$ Pon and starboard oscillatory blowing. $\beta=0^{\circ}, R e_{d}=7400$, $C_{\mu}= \pm 0.0012, \bar{\omega}=0.16$. 
Figure 4.19(b) Port and starboard oscillatory blowing. $\beta=-10^{\circ}, R_{d}=7400$,

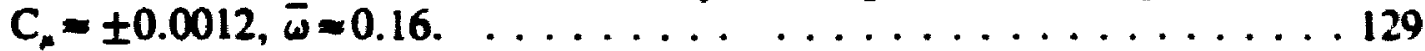

Figure 4.19(c) Port and starboard oscillatory blowing. $\beta=-20^{\circ}, \mathrm{Re}_{\mathrm{d}}=7400$,

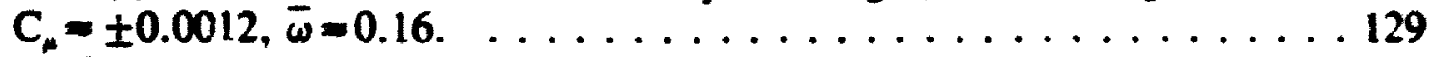

Figure 4.20 Typical stripchart output for oscillatory blowing at a frequency much higher than the maximum a' owable. $\alpha=50^{\circ}, \beta=0^{\circ}, \operatorname{Re}_{\mathrm{A}}=4000$. $C_{A}= \pm 0.0013, \bar{\omega}=0.54$, sensitivity: $0.04 \mathrm{~V} / \mathrm{cm}$, chart speed: $15 \mathrm{~cm} / \mathrm{min}$. .130

Figure 4.21 Nozzle jet exit stream trajectories. Note: Angles shown are approximate. . . . . . . . . . . . . . . . 131

Figure 4.22 Selected F/A-18 fighter aircrart specifications. . . . . . . . 132 


\section{List of Symbols}

\section{English Symbols}

b Span, for this study: d

$b_{6 \pi}$ Wing span of $6 \%$ scale F/A-18 fighter aircraft model.

$C_{n}$ Yaw moment coefficient, $\frac{N}{q . S b}$

$c_{y}$ Side force coefficient, $\frac{\boldsymbol{Y}^{q}}{q_{\mathrm{s}} \mathrm{s}}$

$C_{\mu}$ Coefficient of blowing momentum, $\frac{\dot{m} v_{j}}{q S}$

d Base diameter of forebody and afterbody.

$d_{j}$ Nozzle diameter.

d. Sting diameter.

$d_{6 x}$ Forebody base diameter of $6 \%$ scale F/A-18 fighter aircraft model.

$E$ Young's modulus of elasticity.

$f$ Frequency, in $\mathrm{Hz}$, of oscillatory blowing, or frequency of vortex shedding as applied to Strouhal number.

8 Acceleration due to gravity.

$I$ Moment of inertia, for sting calculations: $\frac{1}{12} d s^{3}$.

$I_{z}$ Moment of inertia about $\mathrm{Z}$ axis of an aircraft, nominally vertical axis through centre of gravity.

$k$ Constant used in forebody parabolic equation.

$L$ Overall length of an aircraft.

$l$ Distance between point of application of side force (centre of pressure) and strain gauges. 
$l_{\alpha}$ Distance betweer centre of pressure and aft strain gauge bridge station.

$l_{\text {Md }}$ Distance between centre of pressure and forward strain gauge bridge station.

$\Delta l$ Distance between strain gauge bridge stations.

l Overall length of forebody.

$\frac{l}{d}$ Slenderness ratio of torebody.

$M$, Bending moment.

$\dot{m}$ Mass flow rate of nozzles jet.

$\dot{m} v_{j}$ Blowing momentum.

$N$ Yaw moment.

N. Yaw moment amplitude.

q. Free stream dynamic pressure, $\frac{1}{2} p V_{-}^{2}$

$R e_{d}$ Reynolds number based on body diameter, $\frac{V_{-} d}{v}$

$\bar{R}_{2}$ Nondimensional radius of gyration of an aircraft.

$r$ Yaw rate, $\frac{d \beta}{d t}$

$r$ Angular acceleration in yaw, $\frac{d r}{d t}$

$S$ Reference area, for this study: $\frac{\pi}{4} d^{2}$

$S_{6 \pi}$ Wing reference area of $6 \%$ scale F/A-18 fighter aircraft model.

ts Thickness of sting at point where strain gauges are mounted.

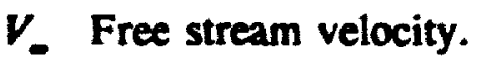

v Nozzle jet exit velocity.

W Weight of an aircraft.

$\boldsymbol{x}_{c}$. Centre of pressure location. 
$x$ Distance along length of forebody aft of apex defining location of nozzles or strakes. Also used as coordinate axis defining forebody parabolic profile.

$\frac{x}{d}$ Nondimensional position of nozzles aft of apex.

$\Delta x \quad X$ coordinate on parabola defining forebody profile.

$\boldsymbol{Y}$ Side force.

y Local radius of forebody corresponding to distance aft of apex.

$\bar{y}$ Nondimensional geometric mean height of various nozzles tested in literature.

Dy Y coordinate on parabola defining forebody profile.

$\frac{z \text { max }}{d}$ Nondimensional width of various nozzles tested in literature.

\section{Greek Symbols}

* Angle of attack.

B Angle of sideslip.

8. Strake deflection angle.

e Strain.

e. Strain at aft strain gauge bridge station.

$e_{\text {md }}$ Strain at forward strain gauge bridge station.

v Kinematic viscosity.

p Density.

- Normal stress.

$\phi_{j}$ Injection nozzle radial position from windward meridian.

1. Forebody roll position of elliptical tip or miniature rotatable nose tip strake. 
申. Strake radial position from windward meridian.

$\omega$ Circular frequency of oscillation.

$\bar{\omega}$ Reduced circular frequency of oscillatory blowing, $\frac{2 \pi f d}{V_{-}}$ 


\section{Introduction}

The flight envelope of highly manoeuvrable aircraft and missiles is undesirably limited due to a lack of controllability at high angles of attack. Characterised primarily by abrupt departures in roll and yaw, this reduced controllability may even lead to unrecoverable, and potentially hazardous, flight modes such as spin. The typical fluid flowfield around a slender forebody at high angles of incidence is dominated by asymmetrically positioned streamwise vortices. Highly manoeuvrable aircraft or missiles operating at high angles of attack encounter yaw control problems caused by these forebody vortices. Vertical tail, or rudder, effectiveness for yaw control decreases with increasing angle of attack because the rudder becomes enveloped in the wake of the wings and body of the aircraft. The rudder thus loses its ability to provide adequate yaw control. At the same time that rudder effectiveness is decreasing, the forces and moments caused by asymmetric forebody vortices are increasing. At high angles of attack these forebody-vortex-generated forces and moments dominate control of the aircraft trajectory and tend to decrease aircraft stability. If vortex orientation can be controlled, then these highly energetic vortices can be used to enhance the performance ( $f$ aircraft and missiles at high angles of attack. Unfortunately, forebody vortex behaviour tends to become erratic with increased angle of attack thereby degrading control potential. This problem must be solved in order to achieve reliable post-stall manoeuvrability. [Cornelius et al, 1992] [Font and Tavella, 1991] [Gittner and Chokani, 1992] [Guyton et al, 1991] [Malcolm, 1991] [Malcolm and Ng, 1991] [Malcolm et al, 1989] [Ng and Malcolm, 1991a] [Ng and Malcolm, 1991b] 
This thesis reports on an experimental investigation into a novel scheme for controlling the behaviour of forebody vortices at high angles of attack. The use of forward blowing from two nozzles, one port and one starboard, located near the forebody apex was investigated. The ability of blowing to 'switch' the asymmetric positioning of the forebody vortices was assessed; moreover the possibility of using relatively rapid alternating, or oscillatory, blowing (ic: port-starboard-port...) to obtain a desired timeaverage side force and yaw moment was investigated. This idea was suggested by Dr. E.S. Hanff of the National Research Council of Canada. Experiments were conducted in a water tunnel test facility. 


\section{Review of Previous Work}

Consider the typical ogive-cylinder model immersed in a free stream fluid shown in figure 2.1. The following will describe the development of the flowfield that surrounds it as its angle of attack is increased. Henceforth, this flowfield will be referred to as the baseline flowfield. It should also be noted that the angles of attack provided in the discussion to follow are approximate values. Actual values are dependant upon model geometry.

At low angles of attack $\left(0^{\circ} \leq \alpha \leq 10^{\circ}\right)$, the flow around the model is vortex-free with no side forces present. As the angle of attack is increased $\left(10^{\circ} \leq \alpha \leq 35^{\circ}\right)$, small longitudinal vortices begin to form on the leeside of the model creating a symmetrical vortex flowfield. In this region of angle of attack, there are still no side forces present. [Malcolm, 1991] [Ng and Malcolm, 1991b]

As further increases in angle of attack are made $\left(35^{\circ} \leq \alpha \leq 65^{\circ}\right)$, the symmetrical flowfield begins to destabilise. The instabilities in the flowfield force the leeward positions of the vortices to change from mirror images to a state of asymmetry in which one vortex is forced into a higher position than the other, see figure 2.1. Earlier boundary layer separation is normally associated with the higher vortex thus the boundary layer separation location of the higher vortex is located more windward than that of the lower vortex. This asymmetric state applies lateral loads on the forebody thereby generating side forces and yaw moments. At this point, the flow is referred to as steady, 
asymmetric vortex flow. [Malcolm and $\mathrm{Ng}, 1991]$ [Malcolm, 1991] [Ng and Malcolm, 1991b] [Moskovitz et al, 1988]

For the higher angles of attack in the asymmetric vortex flow regime $\left(60^{\circ} \leq \alpha \leq 65^{\circ}\right)$, there exists a state in which the flowfield assumes either one of two possible asymmetric patterns such that one vortex is in a higher position than the other. These patterns are mirror images of each other and the slightest disturbance in the flowfield can force the existing pattern to switch into its mirror image. The literature refers to this state as the bi-stable flow regime. [Malcolm, 1991] $\mathrm{Ng}_{\mathrm{g}}$ and Malcolm, 1991a] [Ng and Malcolm, 1991b]

If the angle of attack is increased beyond this region ( $\left.\alpha \geq 65^{\circ}\right)$, the model is at a high enough angle of attack such that the flowfield behaves much like that of a circular cylinder in crossflow. The time-average side forces and yaw moments are then reduced to near zero. [Malcolm, 1991] [Ng and Malcolm, 1991a] [Ng and Malcolm, 1991b]

Figure 2.2 shows the schematic of a generic fighter aircraft as well as the yaw moment behaviour of the baseline flowfields of both the whole model and that of its forebody alone. Note that the additional scale shown in brackets in figures 2.2(b) and (c) . will be discussed in chapter 4 . In both cases, yaw moments caused by an asymmetric vortex flowfield first appear around $30^{\circ} \leq \alpha \leq 35^{\circ}$. As can be seen from the yaw moment characteristics of the entire model, directional stability exists up to $\alpha=30^{\circ}$ (ie: 
$\left.\partial C_{\rho} / \partial \beta>0\right)$. In other words, a restoring moment increment is created to counter the effects of a sideslip increment. This restoring noment is provided by the vertical tail of the model and is referred to as weathercock stability [Etkin, 1982]. Weathercock stability decreases with increasing angle of attack because the vertical tail becomes enveloped in the wake of the wings and body, rendering it ineffective. [Malcolm, 1991] [Malcolm et al, 1989]

The yaw moment characteristics of the forebody alone show that, without a vertical tail, directional stability is negative (ie: $\partial C_{\alpha} / \partial \beta<0$ ). That is, the yaw moment generated serves to compound the effects of sideslip. When comparing the zero sideslip yaw moment characteristics of the entire model to those of the forebody alone, see figures 2.2(b) and (c), it becomes clear that, at high angles of attack, the baseline flowfield is largely determined by the forebody. [Malcolm, 1991] [Malcolm et al, 1989]

Figure 2.3 shows the yaw moment response of the model in figure 2.2(a) to rudder deflections. Again, the additional scale shown in brackets will be discussed in chapter 4. Rudder effectiveness decreases with increasing angle of attack and becomes virtually non-existent beyond $\alpha=40^{\circ}$. At that point, rudder deflections no longer affect the baseline flowfield. Clearly, the rudder cannot be relied upon for directional control much beyond $\alpha=30$ to $35^{\circ}$. The only logical alternative is to investigate methods of manipulating forebody vortices that can potentially create very strong side forces and yaw moments for directional control at high angles of attack. [Malcolm, 1991] [Malcolm at 
al, 1989]

These control methods would operate in the region of asymmetric vortex flow. At this state, the vortices are too large to occupy the leeward side of the body in a naturally stable, symmetric orientation. If the boundary layer separation location of the higher vortex can be delayed, it will react by moving closer to the surface of the model thus forcing the vortex on the opposite side to move farther away. As a result, a net side force and yaw moment is created toward the side with the closer vortex. [Malcolm and Ng, 1991] [Malcolm, 1991] [Ng and Malcolm, 1991a] [Ng and Malcolm, 1991b] [Malcolm et al, 1989]

In order to create an effective forebody vortex control method, it is essential to develop a good understanding of what causes vortex asymmetries. As mentioned earlier, part of the reason is due to the increasing size of the vortices as the angle of attack is increased. Eventually, their interactions force them to adopt asymmetric positions in the flowfield. However, in many cases, the flowfield becomes asymmetric much earlier than expected. Researchers [Malcolm and Ng, 1991] [Malcolm, 1991] [Ng and Malcolm, 1991a] [Ng and Malcolm, 1991b] [Malcolm et al, 1989] [Peake et al, 1980] [Moskovitz et al, 1990] [Gittner and Chokani, 1992] [Moskovitz et al, 1988] generally believed that this was caused by geometric perturbations in the forebodies being used.

In an attempt to determine what effects surface perturbations have on the baseline 
flowfield around a forebody, Moskovitz, Hall and DeJamette (1988), conducted experiments with a single small sphere which they placed at various locations about a nosecone. They discovered that the sphere had the greatest effect on the flowfield when placed radially at a location between 120 and $160^{\circ}$ from the windward meridian. Also, further testing revealed that the sphere diameter required to affect the baseline flowfield decreased as the sphere was moved closer to the tip of the forebody. Thus the flowfield was most sensitive to surface perturbations near the apex. This suggests that a microscopic perturbation, geometric or otherwise, close to the apex can potentially have a global effect on the baseline flowfield. Hence, the careful placement of a perturbation can theoretically reverse the orientation of an asymmetric flowfield.

The remainder of this chapter reviews the most promising methods of forebody vortex manipulation that have been pursued in the available literature. The purpose of this review is to provide the reader with a brief background in these methods of directional control.

The various forebody vortex control methods that are currently being investigated may be categorised as follows:

- Active methods, such as a rotatable elliptical tip.

- Passive methods, such as fixed forebody strakes.

- Pneumatic methods, such as forebody blowing jets or slots. 
- Various combinations of these three methods.

\subsection{Rotatable Elliptical Tip}

Moskovitz, Hall and DeJarnette (1990) have been investigating a device that consists of a rotatable forebody tip which varies in cross section from elliptical at the apex to circular at its base. A schematic of this device is shown in figure 2.4.

Figure 2.5 shows the variation in side force with nose tip roll orientation for the sharp and blunt axisymmetric tips used by Moskovitz et al (1990), respectively. Rows 1 to 3 in the legends refer to the rows of pressure taps on the test model. What is evident here is that the magnitude of the side forces increase with increasing angle of attack and that there is no particular trend in the curves. In other words, the side forces are behaving in their typically raridom nature.

In contrast, when the axisymmetric tip is replaced with an elliptical tip as shown in figure 2.6, the plots become very regular. For angles of attack between 30 and $60^{\circ}$, the side force varies virtually sinusoidally with nose tip roll orientation. It should also be noted that as the ratio of major to minor diameter of the ellipse, or ellipticity, is increased, the magnitude of the side forces increases. This can be seen by comparing the results of tip $\mathrm{O} 1$ with $\mathrm{O2}$. tip $\mathrm{O} 2$ being more elliptic than tip $\mathrm{O}$. This would suggest that the maximum attainable side forces depend on the level of ellipticity. [Moskovitz et al. 1990] 
To determine how effective the rotating elliptical tip would be at providing adequate yaw control, the investigators looked at how well it worked under non-zero sideslip conditions. For a sideslip angle of $10^{\circ}$, a restoring moment was produced for all angles of attack tested. However at a sideslip angle of $15^{\circ}$, a restoring moment could not be produced for angles of attack greater than $40^{\circ}$. [Moskovitz et al, 1990]

\subsection{Strakes}

Strakes are small control surfaces deployed on the forebody that can alter the natural flowfield. The effect that a strake has on the fowfield arunind a forebody is dependant on the local boundary layer thickness and the location of the strake relative to the natural separation line. The natural separation line is determined by the aritude of the forebody relative to the free stream. The primary function of a strake is to control the location of the separation line. The effectiveness of a strake in controlling the location of the separation line is a function of the size, geometry, and position of the strake. [Malcolm and Ng, 1991]

If a strake is placed in an optimum position on the forebody, the location of flow separation can be effectively controlled such that the naturally occurring flow asymmetries associated with leeside vortices can be forced to become symmetric or at least nearly symmetric. Strakes in a fixed position can enhance stability and, if a pair is placed symmetrically, can force vortex symmetry. Additional control can be obtained by deploying strakes actively. [Malcolm, 1991] [Ng and Malcolm, 1991b] [Malcolm et al, 
1989]

Forebody strakes influence the flowfield near the tip of the forebody and this is the region that determines where the vortices will be positioned. A basic understanding of how strakes work can begin by referring to figure 2.7(d). Shorter strakes act as vortex generators, creating small vortices off their trailing edges. These small vortices energise the boundary layer thereby delaying flow separation. Longer, highly swept, strakes affect a large area aft of the apex. Significant portions of the flowfield are forced to separate at the leading edges of the strakes. This action allows longer strakes to produce their own sizable local vorticity. Therefore, longer strakes are more effective in controlling the forebody flowfield over a wider range of angles of attack and sideslip than shorter strakes. [Malcolm and Ng, 1991] [Malcolm, 1991] [Ng and Malcolm, 1991b] [Malcolm et al, 1989]

Strake height affects the overall orientation of the vortices. For small strake heights, the strake does separate the boundary layer but the adverse pressure gradient is not sufficient to maintain the separation. The flow thus reattaches farther downstream before separating again to form a vortex. With the final separation location delayed, the suction pressure increases on the strake side. The vortex on the strake side moves closer to the surface and the vortex on the opposite side is forced to occupy a higher position. The result is a net side force and yaw moment towards the strake. For larger strake heights, boundary layer separation occurs at the leading edge of the strake. This 
separation is strong enough to deter reattachment, therefore the vortex on the strake side moves up and away from the surface and the vortex on the opposite side is forced to occupy a position closer to the surface. The resulting increase in suction pressure on the side opposite the strake creates a net side force and yaw moment away from the strake side. [Malcolm and Ng, 1991] [Malcolm, 1991] [Malcolm et al, 1989]

Experiments on a model with a single strake were performed to determine what effects the radial location, $\phi_{n}$ and deflection angle, $\delta_{1}$, of the strake have on the yaw moments developed by the flowfield [Malcolm, 1991]. Figure 2.8 has a schematic that defines $\phi_{1}$ and $\delta_{1}$ and also shows the variation of yaw moment with strake radial location. The area that proved most effective at changirig the yaw moment was found to be at $\phi_{1}=90^{\circ}$, where the slope is highest. Note that there is a reversal of the yaw moment between $\phi_{1}=60$ and $90^{\circ}$. Figure 2.9 shows pressure measurements from which an explanation of this moment reversal can be obtained. At $\phi_{1}=60^{\circ}$, the strake forces flow separation with the resulting vortex close to the surface. The vortex is followed by reattachment farther downstream which weakens the vortex on the opposite side. A clockwise circulation in the cross flow then occurs which deflects the wake towards the side opposite the strake. Consequently, the suction pressure increases on the strake side and decreases on the opposite side which creates a net yaw moment toward the strake.

At $\phi_{3}=90^{\circ}$. the strake acts like a spoiler pushing the vortex away from the forebody and preventing reattachment. A counter clockwise circulation is created which 
deflects the wake towards the strake. The suction pressure then decreases on the strake side and increases on the opposite side which results in a net yaw moment away from the strake. [Malcolm, 1991]

Changing the deflection angle had similar effects to changing the radial location of the strake. Test results showed that deflecting the strake downstream had the same result as moving the strake radially toward the leeside. Defl ction of the strake upstream produced the same results as moving the strake radially upstream. [Malcolm, 1991]

The radial position, $\phi_{3}$, of a strake has a strcng influence on both the magnitude and direction of the yaw moments. Figure 2.10 clearly shows the importance of $\phi_{\text {. }}$ The addition of strakes at $\phi_{8}=150^{\circ}$, as depicted in figure $2.10(a)$, had a detrimental effect on directional stability. As well, the yaw moments generated are larger than those produced by a forebody without strakes. As the strake radial location is moved more windward, the effects become more beneficial. With the strakes positioned at $\phi_{s}=105^{\circ}$ as shown in figure 2.10(b), the yaw moments generated are nearly zero for all angles of attack. Also, the directional stability is positive for angles of attack up to about $50^{\circ}$. [Malcolm, 1991] [Malcolm et al, 1989]

Overall, for directional control at angles of attack greater than $30^{\circ}$, strakes offer certain advantages. The yaw moments produced by forebody strakes are much larger than those produced by rudders. They are also larger than those produced by the naturally 
occurring asymmetries in the flowfield. Strakes placed in their optimised positions can effectively minimise flow asymmetries. Optimum length, height, radial position and deflection angle of a strake depend on the characteristics of the particular forebody to be modified. In terms of active strake control, single strakes were found to be effective at producing large yaw moments but not very effective at eliminating naturally occurring asymmetries. Dual strakes behaved more satisfactorily. With one strake having a modulating effect over the other, yaw moments can be changed much more gradually. Thus asymmetries can be almost completely eliminated. Experiments indicate that strakes should be deployed in the position that creates the maximum yaw moment on both sides thereby creating a symmetric flowfield. Individual strake heights can then be decreased to produce the yaw moments desired for effective directional control. Further research is required to determine the behaviour of strakes under sicieslip conditions and the effects of varying the Reynolds number [Malcolm, 1991] [Ng and Malcolm, 1991b] [Malcolm et al, 1989]. Also required is an investigation of the effects of strake induced drag.

\subsection{Miniature Rotatable Nose Tip Strakes}

Shown in figure 2.11 , miniature, rotatable nose tip strakes being deflected as a fixed pair provide control similar to regular strakes. Their effectiveness is dependant on angle of attack and the nature of the baseline flowfield. [Malcolm, 1991]

For $30^{\circ} \leq \alpha \leq 60^{\circ}$, symmetric positioning of the nose tip strakes, which is what they will be called herein, results in a symmetrical flowfield with a net yaw moment of 
zero. Rotation of the nose tip strakes changes the vortical flowfield from symmetric to asymmetric with the strake that is closer to the windward side being associated with the vortex that is closer to the body. This results in a net yaw moment toward the strake that is closer to the windward side of the body. As the angle of rotation increases, the asymmetry increases until a maximum attainable yaw moment is reached. This maximum attainable yaw moment varies with angle of attack. For angles of attack greater than 60 , the asymmetries that occur naturally in the flowfield can be increased or decreased by a rotation of the nose tip strakes but their effects are not strong enough to force a symmetrical flowfield. For sideslip angles up to $20^{\circ}$, nose tip strakes are capable of changing the flowfield from destabilizing to stabilizing. [Malcolm and Ng, 1991] [Malcolm, 1991] [Ng and Malcolm, 1991b]

Nose tip strakes function most effectively when the baseline flowfield is near its naturally symmetrical regime. In that region they can easily generate controlled yaw moments. They are capable of providing effective control of vortex asymmetry due to their ability to control separation near the apex by altering the effective geometry of the tip. Compared to the larger strakes discussed earlier, nose tip strakes can be positioned more optimally with respect to the forebody vortices and their separation locations. This makes them effective over a wider range of angles of attack and sideslip. [Malcolm and Ng, 1991] [Malcolm, 1991] [Ng and Malcolm, 1991b]

It was suggested by Malcolm (1991) that more suitable results would have been 
obtained had the port and starboard strakes operated independently.

\subsection{Blowing Jets}

Another method of controlling forebody vortices is by direct injection of fluid into the vortex flowfield using jets from small nozzles. This method alters vortex positions by varying the location of the boundary layer separations. As a result, one vortex moves to a higher position than the other. The higher vortex is always associated with early separation and vice versa. [Malcolm and Ng, 1991] [Malcolm, 1991] [Ng and Malcolm, 1991a] [Malcolm et al, 19891 [Cornelius et al, 1992]

As established earlier, conventional rudder control loses its effectiveness as angie of attack increases but, at the same time, forebody vortex strength increases. Therefore, the potential magnitude of control by blowing also increases. [Malcolm and $\mathrm{Ng}, 1991$ ] [Malcolm, 1991] [Ng and Malcolm, 1991a] [Malcolm et al, 1989]

The first step in studying the effects of forebody blowing jets on the vortex flowfield is to assign a nondimensional coefficient to indicate the level of blowing. In the literature [Malcolm, 1991], this value is referred to as the coefficient of blowing momentum and is defined by :

$$
c_{\mu}=\frac{\dot{m} v_{j}}{q s}
$$


where: $\quad \dot{m}$ is the mass flow rate of the jet,

$v_{j}$ is the velocity of the jet at the nozzle exit,

$q_{\text {. }}$ is the dynamic pressure of the free stream fluid, and

$S$ is the reference area.

It is desirable, naturally, to be able to yield the largest possible yaw moments for directional control with the least amount of blowing momentum. The blowing momentum, $\mathbf{m} v_{j}$, required to cause a switch in the vortices is mainly dependent upon the position of the nozzle aft of the apex of the forebody, the circumferential position of the nozzle, and the angles of attack and sideslip. It is therefore impossible to maintain optimum blowing conditions by using a fixed location for the nozzle jet. Researchers [Malcolm and Ng, 1991] [Malcolm, 1991] [Ng and Malcolm, 1991a] [Malcolm et al, 1989] instead have had to settle on determining a location that has the best overall results for their particular models. This restriction places a limit on the ability to delay the onset of separation thus reducing the maximum attainable vortex asymmetry.

Three types of nozzle jet blowing have been investigated in the literature; blowing normal to the surface, blowing tangentially forward, and blowing tangentially aft.

\subsubsection{Blowing Normal}

Blowing normal to the surface was most effective at low angles of attack. At high 
angles of attack, it did not significantly alter the vortex flowfield. Investigations by Malcolm (1991) and Malcolm et al (1989) revealed that the yaw moment created by normal blowing at low angles of attack was equivalent to the yaw component of the thrust vector created by the blowing. In other words, there were no vortex manipulations taking place and, consequently, the effectiveness of this approach was low. This method will therefore not be discussed further.

\subsubsection{Blowing Tangentially Forward}

To date, the fluid mechanics of blowing tangentially forward are not fully understood. It is known that there are two primary effects. Near the nozzle exit, where the jet velocity is high, the flowfield is dominated by entrainment effects. Entrainment results in reduction of jet velocity. Farther away from the nozzle, the jet velocity is forced to decrease further because of other interactions with the free stream fluid. Eventually, a stagnation zone develops causing separation of the boundary layer. [Malcolm and Ng, 1991] [Malcolm, 1991] [Ng and Malcolm, 1991a]

Effects to the vortex flowfield that result from forward blowing have a strong dependence upon the magnitude of blowing momentum and the angle of attack. At any given angle of attack, the separation location can be significantly altered by variation of blowing momentum. The vortex position, however, remains virtually the same until the blowing momentum is increased sufficiently that the blowing jet reaches the apex of the forebody. At this point, the vortex on the blowing side is lifted away from the surface 
and a net yaw moment away from the blowing side results. [Malcolm and $\mathbf{N g}$. 1991] [Malcolm, 1991] [Ng and Malcolm, 1991a]

Before the blowing coefficient is large enough to allow the jet to reach the apex of the forebody, the response of the flowfield is somewhat different. At low angles of attack, when the matural separation location is normally far leeward the accelerated now caused by these smaller magnitudes of blowing momentum creates a suction zone either directly under or upstream of the vortex. This results in a net yaw moment toward the blowing side. As the angle of attack is increased, the naturally occurring separation line moves windward. Blowing then advances the stagnation zone located aft of the vortex thus promoting earlier boundary layer separation. A net yaw moment away from the blowing side results. [Malcolm and $\mathrm{Ng}, 1991]$ [Malcolm. 1991] [Ng and Malcolm. 1991a]

Figure 2.12 shows the effects of forward blowing from two different nozzle locations. Blowing forward from the more aft location was more favourable, see figure 2.12(b). It provided larger yaw moments for directional control and was more effective over a larger range of angle of attack. The effect that nozzle position has on the baseline flowfield can be observed by inspecting the zero blowing cases. It can also be seen that forward blowing is effective even at low angles of attack. Significant influence to the vortex flowfield occurred for angles of attack as low as $10^{\circ}$. [Malcolm, 1991] [Malcolm et al, 1989] 


\subsubsection{Blowing Tangentially Aft}

Aft blowing functions primarily by controlling the location of flow separation with fluid entrainment. Figure 2.7(a) shows the corresponding flow mechanism. With the commencement of blowing below the higher vortex, fluid entrainment forces the separated boundary layer to reattach. The vortex on the blowing side reacts to this delay in separation by moving closer to the surface and more leeward. At the apex, there exists mutual entrainment interactions between the vortices on both sides of the body. This causes the primary separation location on the side without blowing to advance upstream. The vortex on that side reacts by moving away form the surface and more windward. The end result is a reversing of the flowfield asymmetry and thus a net yaw moment toward the blowing side is created. It should also be noted that blowing under the lower vortex results in an enhancement of the existing asymmetry and therefore an increase in the net yaw moment toward that side. [Malcolm and Ng, 1991] [Malcolm, 1991] [Ng and Malcolm, 1991a] [Malcolm et al, 1989] [Guyton and Maerki, 1992]

Experiments [Malcolm, 1991] [Peake et al, 1980] to determine the impact that nozzle circumferential position has on the effectiveness of aft blowing revealed that yaw control can only be accomplished when the jet is on the leeside of the body. This is in agreement with the surface perturbation tests discussed earlier. Locating the nozzle between 120 to $150^{\circ}$ from the windward meridian placed the nozzle in the region where entrainment of the injected fluid into the separated boundary layer was optimised. INg and Malcolm, 1991a] [Gittner and Chokani, 1992] 
It was also determined that aft blowing was most effective when the nozzle was positioned as close to the apex as practicality would allow. Aft blowing can then control the flowfield from its most forward location where the region of mutual entrainment interactions exist. [Malcolm and Ng, 1991] [Ng and Malcolm, 1991a] [Comelius et al, 1992] [LeMay et al, 1992] [Gittner and Chokani, 1992]

At very high angles of attack, in the area referred to as the bi-stable region [Malcolm, 1991], larger magnitudes of momentum blowing were required to switch the asymmetric positions of the vortices. Once the vortices were switched, turning off the jet did not result in the vortices returning to their original asymmetry. Instead, the vortices stayed in their new positions with only a slight decrease in the degree of asymmetry. Therefore, while in this bi-stable region, the vortex flowfield can be switched between two stable asymmetric states that are mirror images of one another. [Malcolm and Ng, 1991] [Malcolm, 1991] [Ng and Malcolm, 1991a]

Simultaneous port and starboard blowing aft served to strengthen the vortices on both sides and pull them down closer to the surface. Thus it augmented the stability of the symmetric flowfield and delayed the onset of asymmetry. However, the slightest perturbation in the flowfield had strong effects on the stability of this symmetric state. This suggests that trying to maintain a symmetric flowfield by symmetric blowing is impractical. [Guyton et al, 1991] [Malcolm, 1991] [Malcolm et al, 1989] 


\subsubsection{Effects of Nozzle Exit Geometry}

Gittner and Chokani (1992) conducted experiments to determine the effects of nozzle jet exit geometry on the performance of aft blowing. Figure 2.13 shows the various configurations tested. Their analysis showed that, without blowing, the boundary layer separation advanced on the side where the nozzle was located. Blowing effectiveness was gauged by measuring the surface pressure along the length of the forebody with a sustained suction pressure being associated with improved blowing effectiveness. It was determined that lowering the geometric centre of the exiting jet allowed blowing effectiveness to be maintained farther downstream. Below a certain point, further reductions in geometric centre showed no sign. ficant improvement in blowing effectiveness. Also, broader exit nozzles such as the onss with elliptic cross sections (ie: nozzles 1 and 3 in figure 2.13) were more effective than the narrower nozzles.

Comelius et al (1992) also conducted research into the effect of nozzle exit geometry. The various nozzle geometries, shown in figure $2.14(a)$, were converging/diverging (C-D), axisymmetric (A1), and slotted (S1). Experiments were conducted at pressure ratios ranging from 20 to 30 (nozzle total pressure/tunnel static pressure). A schematic defining the orientation of the nozzle axis relative to the body of the model is shown in figure 2.14(b). The largest yaw moments were generated using the slotted nozzles with the slots positioned in the vertical plane. Slot length had a minor effect but slot width appeared to be the important parameter. Careful control of slot 
width allowed the spread angle of the exiting supersonic flow to expand in a two dimensional sheet thereby yielding greater aerodynamic interaction between the jet and the flowfield around the forebody. [Comelius et al, 1992] [Guyton and Maerki, 1992]

\subsubsection{Pulsed Blowing Jets}

A time lag exists for the net yaw moment to be altered by a blowing jet. A pulsing jet with a period shorter than that time lag would eliminate the requirement for a steady stream of injecting fluid. Research with an aft blowing pulsed jet [Malcolm and Ng, 1991] showed that, for low pulsing frequencies, the frequency of oscillation of the vortex on the blowing side was proportional to the frequency of the pulsed jet and that the rest of the flowfield responded accordingly. As the frequency of the pulsing jet increased, the vortex pattern steadied until the vortices on both sides locked into fixed positions. With a high frequency jet pulse, the separation on the blowing side responded almost immediately, while the separation on the opposite side and the vortices on both sides remained virtually in the same position. Due to the existence of time lag effects in the flowfield, both vortices and the separation on the side without blowing cannot respond fast enough to the changes in the position of the separation on the blowing side. Therefore the net result of increased frequency pulsed jets is that the vortices on both sides as well as the separation on the no blowing side become steady due to the time lag effects even though the separation on the blowing side is oscillating at the blowing frequency. [Malcolm and Ng, 1991] [Ross et al, 1991] 


\subsubsection{Aft and Forward Blowing Combined}

With a forward blowing nozzle positioned on one side of a forebody and an aft blowing nozzle positioned on the opposite side, as figure 2.15 shows, unexpected results occurred. Note that this test also included strakes positioned on the forebody. The combination of strakes and nozzle jet blowing will be discussed later. The response of the flowfield to forward blowing alone was virtually the same as discussed earlier. As well, with aft blowing alone, the flowfield behaved as expected. In short, the resulting yaw moments were opposite one another. However, when blowing both forward and aft simultaneously, the yaw moment produced was not the resultant of two vectors as one would expect. Rather, the effects of forward blowing were enhanced by those of aft blowing. The additional yaw moment was approximately equal to that which results form aft blowing alone but in the opposite direction. This indicates that an extremely powerful synergistic effect exists. Further investigations into this combination revealed that forward blowing was the dominant mechanism with aft blowing always enhancing the effects of forward blowing. The only exception was when forward blowing was kept at a very low magnitude. At that point it was aft blowing whose effects dominated. Thus it can be said that the yaw moments produced by simultaneous forwand and aft blowing exceeded the magnitudes obtained by either method alone. [Malcolm and Ng, 1991] [Malcolm, 1991] [Ng and Malcolm, 1991a] [Malcolm et al, 1989]

\subsection{Strakes and Tangential Blowing Combined}

It is very difficult to maintain a zero net yaw moment with either symmetric or 
asymmetric blowing jets. Results suggest that the best use for momentum blowing is on a model that has a naturally symmetric or slightly asymmetric vortex flowfield for all angles of attack and to perturb the flow away from this state to generate controlled yaw moments [Malcolm et al, 1989] [Gittner and Chokani, 1992]. As previously discussed, symmetric nozzle jet blowing produced a highly unstable vortex symmetry that was very sensitive to minor perturbations in the flowfield. It was also established that the symmetric positioning of forebody strakes was extremely successful at maintaining a symmetric flowfield. Therefore, a good idea for yaw control using forebody vortex manipulation would be to create a symmetric flowfield using strakes and then to generate controlled asymmetries in the flowfield by tangential blowing. The addution of forebody strakes reduces the sensitivity of the flowfield to blowing. If the strakes are large enough, the symmetric flowfield becomes too stable. This makes it more difficult to perturb the vortices requiring larger magnitudes of momentum blowing as input. [Malcolm and Ng. 1991] [Malcolm, 1991] [Malcolm et al, 1989] [Cornelius et al, 1992]

Figure 2.16 shows the results of combining symmetric forebody strakes and aft tangential blowing. Blowing with this configuration successfully alters the flowfield so as to produce a yaw moment towards the blowing side. The port and starboard blowing results were near mirror images of one another. The yaw moment produced by a $30^{\circ}$ rudder deflection was also plotted for comparison. Note how blowing starts to produce significant yaw moments at approximately the same point that the rudder becomes ineffective. Researchers [Malcolm and Ng, 1991] [Guyton et al, 1991] [Malcolm, 1991] 
[Malcolm et al, 1989] also reported that this configuration is effective for sideslip angles up to $20^{\circ}$ with blowing gencrating a large enough yaw moment to counter the negative directional stability that occurs naturally at high angles of attack.

Blowing tangentially forward on a model with forebody strakes produced results that were less desirable. At low magnituces of momentum blowing, the yaw moments produced were in the direction away from the blowing side. When the blowing rate was increased, the yaw moment switched directions and acted toward the blowing side. It should be noted that these results are opposite to those provided earlier in this chapter for forward blowing withoit strakes as described by the same authors. The fluid mechanics that cause this switch in direction are not fully understood at this point. Suffice to say that this characteristic makes forward blowing less desirable than aft for such an application. [Malcolm, 1991] [Malcolm et al, 1989]

Simultaneous blowing differentially either forward or aft was performed by applying the maximum blowing rate on both sides of the forebody and then varying only one side for control. Compared to single sided blowing, differential blowing on both sides offered finer adjustment capability. [Malcolm, 1991] [Malcolm et al, 1989]

Researchers [Guyton et al, 1991] [Cornelius et al, 1992] [Guyion and Maerb. 1992] have been altempting to optimise the configuration of combining strakes with aft tangential blowing. This has lead to an investigation into the effects of cant angle. Cant 
angle is defined in figure 2.14(b). Providing aft facing nozzles with an outward cant angle only served to confirm the findings of Malcolm (1991) and Malcolm et al (1989). Namely that the yaw moment produced was due to the component of jet momentum in that direction. In other words, the blowing jets ended up acting like directional thrusters. Inward canting proved more effective. Providing aft facing, slotted nozzles an inward cant angle of $60^{\circ}$, as shown in figure $2.14(\mathrm{~b})$, resulted in the improvements to the flowfield shown in figure 2.17. Figures $2.17(\mathrm{a})$ and (b) show schematically the baseline flowfield and the flowfield resulting from blowing tangentially aft respectively. Figure 2.17(c) shows the schematic of the optimised configuration shown in figure 2.14(b). The vortex on the blowing side has now moved even closer to the surface and more leeward to the plane of symmetry of the model. This results in an increase in the amount of attached flow. The vortex on the opposite side has not only moved more windward, it has now moved far away from the surface. Lastly, the flow from the blowing side becomes entrained into the primary vortex separation on the opposite side. The result of this optimised configuration is a ten fold increase in the magnitude of the yaw moments produced over those generated by the same arnount of aft tangential blowing. [Guyton et al, 1991] [Corne:ius et al, 1992] [Guyton and Maerki, 1992]

\subsection{Blowing Slots}

Tangential slot blowing functions on the principles of circulation control. As depicted in figure $2.7(\mathrm{~b})$, the fluid emerging from the slot energises the flow near the surface. This action permits the flow to overcome any adverse pressure gradients it may 
encounter thereby delaying boundary layer separation on the blowing side. This effect increases with increasing blowing rate. Consequently, the vortex on the blowing side moves closer to the surface and more leeward while at the same time the vortex on the opposite side moves away from the surface and more windward. The net side force and yaw moment that result from this mechanism are toward the blowing side. [Malcolm and $\mathrm{Ng}, 1991$ ] [Malcolm, 1991] [Ng and Malcolm, 1991a] [Murman et al, 1992]

As was the case with blowing jets, an optimum relation between slot location and separation zone control cannot be maintained over the entire manoeuvring envelope. In fact, blowing effectiveness is strongly dependant on slot axial location. This is due largely to the existence of significant variations in local flow conditions aiong the length of the slot. In addition, as these local flow conditions vary more near the apex than they do downstream, a slot placed in a forward position is more prone to this effect than one placed farther aft. [Malcolm and Ng, 1991] [Malcolm, i991] [Ng and Malcolm, 1991a]

Blowing tangentially from slots placed in an aft position on a forebody was found to be effective at controlling the local flow separation on the blowing side. They failed to affect regions upstream of the slot location closer to the apex. Therefore, they did not have any effect on the flowfield where mutual entrainment interactions are strongest. [Malcolm and Ng. 1991] [Malcolm. 1991] [Ng and Malcolm. 1991a] [Font and Tavella. 1991] 
Tangential bluwing from slots positioned near the apex affected the flowfield where interactions between port and starboard sides are strongest. This action had a strong effect on vortex trajectory. The disturbances created in this area propagated downstream along the length of the vortex. Thus, from an aerodynamic perspective. tangential blowing from a slot positioned near the apex was more effective at producing controlled yaw moments than blowing from a slot positioned farther aft. For this configuration. control starts becoming effective at angles of attack as low as $30^{\circ}$, and $\mathrm{uds}$ found to remain effective for angles of sideslip as large as $20^{\circ}$. [Malcolm and $\mathrm{Ng}, 1991$ ] [Malcolm, 1991] [Ng and Malcolm, 1991a]

Careful control of the blowing rate should be maintained. If it is increased too high, the blowing sheet separates from the foretody immediately at the slot. The shear layer then becomes unstable and rolls up into individual vortices that interact with the forebody vortices downstream. This results in a loss in control effectiveness. I $\mathrm{Ng}$ and Malcolm, 1991a] [LeMay et al, 1992]

\subsection{Suction}

Three methods of forebody suction have been investigated; slots, holes, and suction through a porous surface. The fluid mechanics of forebody suction, shown in figure $2.7(c)$, operate under the principles of circulation control. Highly energetic boundary layer flow is maintained by pulling the high speed free stream flow closer to the surface. The resulting delay in separation of the boundary layer creates a net side 
force and yaw moment toward the suction side. The suction rate required to affect large forebody vortex structures is relatively low. Control starts to take effect at angles of attack near $25^{\circ}$ and remains effective for angles of sideslip up to about $20^{\circ}$. [Malcolm and $\mathrm{Ng}, 1991]$ [Malcolm, 1991] [Ng and Malcolm, 1991a]

All three suction methods were most effective when placed closest to the apex near the leeside of the forebody. Suction through a porous surface had the lowest suction rate requirements and was found to be effective over a larger range of angle of attack and sideslip than suction through either slots or holes. This was due to the fact that the porous surface was spread out over a wider area and therefore remained in an optimum control position over a larger manoeuvring envelope. [Malcolm and Ng, 1991] [Malcolm, 1991 ] [Ng and Malcolm, 1991a]

Surtior effectiveness was found to depend upon nose apex angle, length to diameter, or slenuemess, ratio, and angles of attack and sideslip. [Ross et al, 1991]

\subsection{Overvie*v of Control Methods}

All of the .....hods discussed operate on the principle of forced vortex flowfield symmetry or biased asymmetry. The most successful controls are obtained by altering the flowfield near the apex of the forebody thereby modifying the effective geometry of the nose tip region. [Malcolm and $\mathrm{Ng}$. 1991] [Malcolm, 1991] [Ng and Malcolm, 1991b] 
The blowing and suction rates required to affect the flowfield are small enough that engine bleed on an actual aircraft would be adequate. [Malcolm and $\mathrm{Ng}, 1991$ ] [Malcolm, 1991] [Ng and Malcolm, 1991a] [Malcolm et al, 1989]

As previously discussed, with increased angle of attack, forebody vortex control effectiveness increases while vertical tail effectiveness decreases. Thus it can be concluded that. a: Ic: angles of attack, directional control can best be accomplished by aerodynamic surfaces aft of the centre of gravity of an aircraft. However, at high angles of attack, this task would best be served by aerodynamic devices forward of the centre of gravity close to the apex of the forebody. [Malcolm. 1991]

\subsection{Transient Behaviour of the Lateral Loads}

The side forces and yaw moments that result from the various forebody vortex manipulation methods exhibit a transient and temporal behaviour. Figures 2.18(a) and (b) show the highly unsteady nature of the yaw moment for an aft blowing jet and for a miniature rotatable nose tip strake rotation respectively. A transient overshoot in yaw moment exists in response to the perturbations created by imposing or removing the jet or rotating the nose tip strakes. At low blowing rates, this overshoot is generally greater than the average yaw moment created. For larger blowing rates however, the overshoot was found to be less than the average yaw moment. [Malcolm and $\mathrm{Ng}, 1991$ ] [Malcolm, 1991] [Ng and Malcolm, 1991a] [Ng and Malcolm, 1991b] 
Although not evident in figure 2.18 . Malcolm (1991) observed that blowing had a stabilising effect on these fluctuations which reduced their magnitudes.

\subsection{Proposed Method to Alleviate Lateral Loading}

All the methods of forebody vortex manipulation discussed here have concentrated on trying to create controlled stead lateral loads to counter the naturally occurring asymmetries in the baseline flowfield at high angles of attack. Consequently, all of these methods have had to endure the random fluctuations inherent in the baseline flowfield. Rather than attempting to generate steady lateral loads, this study sets out to devise a method using tangential blowing jets to create a rapid transfer of forebody vonices such that the time average of the side forces and yaw moments is zero. What would happen to the random fluctuations under these conditions remains to be seen but it is expected that they will become superimposed onto the induced oscillations. It is conceivable that this method could also be used to create non-zero time-average side forces and yaw moments by applying either different blowing rates between port and starboard nozzles or oscillatory blowing with an asymmetric time delay. However, this possibility will not be investigated in this study. 


\section{Description of Apparatus and Experiments}

Experiments were conducted in the water tunnel at Carleton University. At the start of this project, the water tunnel had not seen substantial use for several years. Consequently, a great deal of maintenance and repair work was required to get it up and running again. Even now, there are systems on the water tunnel that are not operational since they were not needed for the present experiments. This chapter contains a detailed log of the repair work done in order to prevent any duplication of effort and to provide future researchers with the benefit of the author's experience and a ready reference for troubleshooting.

\subsection{Water Tunnel}

The stainless steel water tunnel, shown in figure 3.1, consists of a closed loop. in a vertical plane, with a $12 \times 12 \times 53$ in $(0.3 \times 0.3 \times 1.3 \mathrm{~m})$ test section. The te: : ssction consists of four $2 \mathrm{in}(5 \mathrm{~cm})$ thick plexiglass panels mounted onto a thin support frame. The top panel has two squa 2 holes, one located upstream and the other located downstream, to allow access into the test section. The smaller upstream hole is used to mount a dye rake assembly while the larger downstream hole accommodates the model assembly. Both filling and draining of the water tunnel take place through the holding tank. The top leg of the water tunnel can be completely drained into the holding tank so that work may be performed on the test section without wasting any water. Once the work is completed, the water in the holding tank can be pumped back into the test section to resume experiments. Undesired water can be discarded from the holding tank into a sump located 
below the floor of the laboratory through a drain valve mounted at the bottom of the holding tank.

Several leaks, some minor and some major, became apparent the first time the water tunnel was filled. The most serious of these leaks was at the housing that held the bearing and seal for the impeller drive shaft. Dismantling of the assembly on the outside of the water tunnel and removal of the porthole on the bottom leg of the tunnel revealed that both inner and outer bearings and the accompanying seals required replacement. The impeller shaft itself was worn in some areas, but the damage was not so severe as to merit its replacement. The bearing, seal, and accompanying assembly outside of the water tunnel were replaced with new components. Replacement of those located inside the water tunnel was not performed as it would have required complete disassembly of the downstream portion of the water tunnel. Replacing the outer bearing assembly and keeping the new bearing tightly packed with grease did temporarily stop the leak. However, after several months of experimenting, the leak has started again and rotation of the impeller shaft creates more noise than it did in the past. For this reason, it is strongly recommended that the entire impeller drive shaft assembly, both inside and out be overhauled. Also, it was observed that the impeller itself was severely corroded and pitted, thus it may require replacemen: in the near future.

Other leaks that required immediate attention were located on the top of the test section. The o-ring that formed the seal at the dye rake assembly had dried up so a new 
one was purchased and installed. The o-ring that sealed the model-support insert panel was missing, see figure 3.2. Since this o-ring was not a standard size, a kit was purchased and a new o-ring was made. Unfortunately, during installation, it was discovered that the slot which housed this o-ring was machined too deep resulting in zero deformation of the o-ring and consequently, no seal. It was decided that a thin gasket would be used to line the bottom of the slot thereby elevating the o-ring and allowing it to be deformed by the model assembly thus forming a seal. The original intent was to use this gasket as a temporary measure in the hope that it would at least reduce the leak to a tolerable level. As it turned out the seal was completely water proof and has held up very well so no further modifications to this seal are necessary.

The model-support insert was fastened directly to the main plexiglass ceiling panel of the test section by two bars that were clamped down by four support blocks. These support blocks were screwed directly into the plexiglass thus, over the years, overtightening of the clamps resulted in one of the blocks occasionally breaking off along with a portion of the plexiglass. By the time this project was started, all four blocks had been broken off and re-glued onto the surface of the plexiglass. To prevent this problem from recurring, the clamping blocks were removed and replaced by two lengths of angle iron that are bolted directly into the steel frame of the test section, see figure 3.2. The loads on the model-support insert are now transferred to these angle iron bars via two backing bars and adjustable bolts, as shown in figure 3.2 . 
There were various other minor leaks all around the water tunnel, mostly at the flanges. These were repaired with silicone sealing compound. These repairs only lasted about two or three weeks so more silicone had to be applied each time the water tunnel was drained. Although not a difficult task, future researchers may nevertheless want to find a more permanent solution.

The water tunnel impeller can be driven by either one of two electric motors, the choice depending on the desired operating speed. The larger, $40 \mathrm{Hp}(30 \mathrm{~kW})$ motor is permanently coupled to the impeller shaft by a belt and pulley assembly. It provides the high and intermediate operating speed ranges. The smaller, 1/4Hp (190W) motor that provides the low speed range of operation slides on a tray into position and transmits torque to the impeller shaft through a gear box and a pair of clutch plates, see figure 3.3. The switches to select the drive range and the rheostats used to set the shaft speed are located on the tunnel control panel, which is beside the holding tank, see figure 3.1.

Preliminary testing of the motor drives revealed that the high speed range did not work properly. The motor accelerated to an unacceptably high speed as soon as the rheostat was touched. Since this operating range was not required for this project, the necessary repair work was not performed. It should be noted that future researchers need not effect repairs to the circuitry that controls this operating range unless they plan to do cavitation testing as the speeds available are quite high. The intermediate speed range worked without problems; adjustment of the rheostat delivered smooth steady torque to 
the impeller shaft but the speed of the shaft increased slightly as the motor warmed up. The low speed drive motor worked reasonably well but it was found that its output was not very steady and the clutch plates were so badly worn that they would not engage. A new set of clutch plates, shown in figure 3.3, were machined and after installation proved to work very successfully. Also, it was found that the fluctuations in the output shaft steadied as the motor was allowed to run. It is believed that this unsteadiness in shaft rotation is due to impeller shaft bearing friction because when the clutch was disengaged, the motor shaft rotated without fluctuations. Therefore, in general, it is recommended that the water tunnel be run for about 10 to 20 minutes before experimenting to allow the free stream velocity to become constant regardless of which speed range is being used.

Referring back to figure 3.1, a design flaw in the water tunnel was discovered while the repair work was taking place. During filling of the top leg of the water tunnel, a large air bubble would become trapped in the diffuser downstream of the test section, a local high-point in the circuit. As the water tunnel operated, the free surface of the water under this bubble would undoubtedly change shape and perhaps volume, causing fluctuations in the free stream velocity despite the constant speed of the impeller shaft. A hole was drilled and a quarter-tum ball valve installed at the highest point downstream of the test section so that air can now be bled out while the tunnel is being filled.

While filling the water tunnel for the first time, it was discovered that the bottom leg was never completely drained. Note that the existing drain valve is situated at the 
lowest point in the bottom circuit of the water tunnel. There was no apparent reason for the bottom leg of the water tunnel to have been left filled with water; presumably the previous users just forgot about it. The point being that no modifications in the drainage system of the water tunnel are required. Simply, future users should adopt the habit of leaving the drain valve open when the tunnel is not in use. As a consequence, the impeller and shaft were submerged for a long period of time. Earlier, it was mentioned that the impeller was badly corroded and pitted. In fact, the first few test runs resulted in sizable pieces of loose rust settling at the bottom of the holding tank. It became evident that the water tunnel had to be cleaned and an appropriate additive to maintain water clarity had to be administered. A likely candidate for these tasks was the chlorine used to maintain clarity and sanitary conditions in swimming pools. The only uncertainty was what effects, if any, would such a chemical have on the stainless steel walls of the water tunnel. To find out, several small samples of stainless steel were placed in a highly concentrated solution of chlorine and water for one week. The samples were then removed and allowed to dry for two days. Corrosion took place only on the surfaces that were freshly cut to create the samples. It is believed that corrosion took place in those areas because they were not allowed to oxidise sufficiently before being submerged in the concentrated solution. Thus it was decided that the use of swimming pool chlorine would not have any significant corrosive effects on the walls of the water tunnel.

The water tunnel was flushed as follows: About 33lbs (15kg) of calcium hypochlorite, a dry granular chlorine (supplier and brand name are listed in Appendix 
A), was dissolved in water in the holding tank and pumped into the water tunnel. This compound is not recognised specifically as a rust cleaner, hut it is known to be an effective algicide and was found to be mildly abrasive. The water tunnel was then run at the highest speed available from the intermediate drive for about one hour and then the concentrated solution was replaced by fresh water. The fresh water was circulated in the water tunnel for another hour before being drained into the sump. As there were virtually no loose rust deposits left at the bottom of the holding tank, it was assumed that the water tunnel was now sufficiently cleaned.

To maintain water clarity, about 0.1 ounces ( 3 grams) of the granular chlorine used to flush the water tunnel were added to the holding tank every two days. Water with this concentration of chlorine could remain in the water tunnel indefinitely or until dye from flow visualisation experiments significantly clouded the water. When changing the water, it is recommended that the supply water be allowed to pass through the holding tank into the laboratory sump until the stagnant water in the building supply lines is replaced with fresh water. Doing so provides the water tunnel with clearer water at the start. To prevent air that is dissolved in the fresh water from settling as bubbles on the model and the walls of the test section, the water should be allowed to sit in the holding tank for 24 hours before initially filling the test saction. Note that the lower half of the tunnel circuit would be filled with water during this time but air bubbles settling on the walls of the water tunnel in that area are of no consequence. Also, water for the test section should be kept in the holding tank when testing is not taking place. For reference, 
the water tunnel can be completely drained four times before the laboratory sump has to be emptied. Emptying the sump involves pumping out its contents through a fire hose into the municipal sewer lines.

\subsection{Test Equipment}

The test equipment was designed with the philosophy of creating as many generic components as possible so that they may be used on future projects with little or no modification. The model consisted of an ogive-type forebody attached to a cylindrical afterbody and mounted onto a sting. The size of the test section and the requirerient of high angle of attack deflections limited the model to a 1.0 in $(2.54 \mathrm{~cm})$ diameter and about 6in $(15 \mathrm{~cm})$ in length. Plexiglass was chosen as the material for the model because it was readily available, easy to machine, corrosi $\cdot n$ resistant, and transparent.

A parabolic meridional profile was selected for the forebody with the criterion of a $60^{\circ}$ included nosecone angle at the apex and a diameter of $1.0 \mathrm{in}(2.54 \mathrm{~cm})$ occurring 2.0 in $(5.08 \mathrm{~cm})$ aft of the apex. The manufacturing drawing for the nosecone is shown in figure 3.4. Tangency between the forebody and afterbody was accomplished by machining a radius in blend about $1 / 16$ in $(1.59 \mathrm{~mm})$ forward of the forebody-afterbody junction, see figure 3.4 . The resulting slenderness ratio was $\ell / d=2.25$. The parabolic portion of the forebody profile is defined by:

$$
y=\sqrt{\frac{(x+0.1653)}{4.5359}}-0.1909
$$


where: $\quad x$ is the distance along the length of the forebody aft of the apex, and $y$ is the local radius of the forebody corresponding to the distance. $x$, aft of the apex.

The nosecones were manufactured by producing a template on a CNC milling machine and then using this template with a copying attachment on a precision lathe. Five torebodies were machined and during comparisons on a shadowgraph, repeatability between dimensions was always within \pm 0.0005 in $(0.013 \mathrm{~mm})$. Figure 3.5 shows the various nosecones used during the course of these experiments.

To achieve a rapid transfer of forebody vortex orientation, the easiest of the control methods cited in chapter 2, from a manufacturing perspective, was a pneumatic approach, either blowing or suction. Blowing was chosen over suction since, with the setup that existed on the water tunnel, suction would have irad a more limited range of mass flow rate as compared with blowing. Size restrictions ruled out any possibility of bending the tubes used to make the nozzles. Also, it was desirable to mount the nozzles flush with the surface rather than having them stick out. For those reasons, forward blowing was selected over aft. Stainless steel hypodermic needles were used to make the nozzles. Experiments were conducted on forebodies with nozzles at two different axial locations, see figure 3.5. The nozzle-opening diameter used at $x / d=1.0$ was 0.045 in $(1.14 \mathrm{~mm})$ while that used at $x / d=0.25$ was $0.020 \mathrm{in}(0.51 \mathrm{~mm})$. As shown in figure 3.5 , fluid was delivered to the nozzles by holes that were drilled into the nosecones. The hypodermic needles were not deformed in any way, rather, they were pressed into the 
supply passages drilled in the plexiglass forebodies. After mounting, the nozzle exits were machined flush with the forebody surface on a lathe, see the inset in figure 3.4. Supply lines were connected to a pair of brass tubes that were inserted at the base of each forebody. The nosecones were secured to the cylindrical afterbody by an o-ring, with a light press fit, making them very easy to replace and align, see figure 3.5. The afterbody consisted of a hollow cylindrical plexiglass tube that was mounted onto the sting by a threaded disk located at the nosecone-afterbody interface. The afterbody was hollow to allow passage of the blowing supply lines. The complete model assembly is shown in figure 3.6.

It was intended that experiments were to be conducted at relatively low speeds. The free stream velocities for flow visualisation and force measurement tests were to be about $3 \mathrm{in} / \mathrm{sec}(7.6 \mathrm{~cm} / \mathrm{sec})$ and $12 \mathrm{in} / \mathrm{sec}(30.5 \mathrm{~cm} / \mathrm{sec})$ respectively. It was therefore anticipated that the side forces generated would be rather small and consequently, the balance had to be made of a material with a low enough Young's modulus of elasticity such that the strains generated by the aerodynamic loads would be large enough to be detected by the strain gauges. For this reason, the original material selected for the balance and sting was polyvinylchloride (PVC). This was changed to aluminum fur reasons that will be discussed later. The purpose of the sting was to connect the mode! to the attitude control mechanism, see figure 3.6 , and, when instrumented with strain gauges, to aci as a force and moment balance to measure lateral lmading. Made from $1 / 4$ in $(6.4 \mathrm{~mm})$ diameter rod, the sting had flat surfaces machined on opyusite sides at 
two axial stations. The remaining material between the flats was in a vertical plane and was $1 / 16$ in $(1.6 \mathrm{~mm})$ thick, giving a high sensitivity to side forces and virtually no sensitivity to lift forces, see appendix B. A strain gauge was mounted on each flat surface and the pairs opposite one another were wired to form one half of a Wheatstone bridge [Perry and Lissner, 1955]. The full Wheatstone bridge circuits were completed by connecting each pair of strain gauges to a strain indicator. This wiring scheme will be discussed in more detail later on in this chapter. The ability to measure bending moments on the sting at two stations made it possible to deduce both side force and yaw moment acting on the model.

The model-sting assembiy is attached to an attude control mechanism which is in turn fastened to the plexiglass mudel-suppon insert shown in figure 3.2. The attitude control mechanism consists of two concentric shafts and a linkage, see figure 3.6. The inne: shaft, which is the longer of the two, is threaded. Rotation of the inner shaft forces the linkage to move thereby causing the model to pitch. Rotating the outer shaft conirols the yaw angle of the madei. Adjustment of both pitch and yaw angles can be done with the water tunnel running since the controls, located ahove the test section, are sealed with o-rings, see figure 3.2 . In order to have an unobstructed view of the top of the model, the inodel and sting assembly are nicisnted inverted onto the attitude control mechanism. Pitch can be adjusted between $\alpha \approx 10$ and $70^{\circ}$. Zero angle of attack is not possible with the present configuration because of size restrictions. Pitch indication is achieved by a 
protractor mounted at the base of the sting inside the test section. The yaw angle can be adjusted between $\beta \approx \pm 20^{\circ}$ and is indicated by a protractor mounted on top of the modelsupport assembly outside the test section. The most attractive feature of the attitude control mechanism is that the model and sting assembly can be easily removed so that in the future, another assembly can be installed in its place.

Figure 3.7 shows a schematic of the plumbing system used for nozzle jet blowing and flow visualisation. One of the three pressurised dye reservoirs delivers red coloured fluid to the upstream dye tube while the remaining two enable either clear or blue coloured fluid to be sent to the injection nozzles on the forebody of the model. The reservoirs are made of plexiglass cylinders and are pressurised to a maximum of 3 psi (20.7kPa). Initially, the dye reservoirs were mounted on a platform located below the test section, above the bottom leg of the water tunnel. Although the maximum attainable nozzle jet velocity was acceptable for flow visualisation with the dye reservoirs in that position, forebody vortex manipulation required higher velocities. Since the dye reservoirs were already being charged to their maximum allowable pressure, the only other alternative was to elevate them. The dye reservoirs are now located downstream of the test section on a platform mounted above the top leg of the water tunnel, see figure 3.1. Manually adjusted needle valves regulate the flow of fluid out of the dye reservcirs so both nozzle jet blowing and flow visualisation can be accomplished with the reservoirs in their new position. 
Flow visualisation was performed by injecting coloured water through the nozzles in the forebody and through an upstream dye tube. Diluted stamp-pad ink was used to colour the water. On average, about 20 drops of stamp-pad ink were required per reservoir to produce the desired colouring. The upstream dye tube, made of stainless steel tubing with an oute: diameter of $1 / 16 \mathrm{in}(1.59 \mathrm{~mm})$ and an inner diameter of $0.045 \mathrm{in}$ (1.14mm), delivered red dye into the free stream fluid when desired. Blue dye could be fed to the forebody nozzles so that the injected fluid may be followed as it travelled downstream. The interaction between the red and blue coloured fluids revealed how the injected fluid interacted with the free stream fluid as it passed over the forebody. The lighting used to capture the fluid flow on film consisted of three 200 watt incandescent bulbs mounted inside a portable aluminum box. The box was positioned behind the back wall of the test section so that profile pictures of the model could be taken without the occurrence of shadows. The light was diffused by a sheet of translucent mylar that was pasted directly onto the plexiglass wall of the test section. A $\operatorname{lin}^{2}\left(6.5 \mathrm{~cm}^{2}\right)$ grid was drawn on the mylar so that a ready size reference was aluays available for photographing and filming. Although this system of lighting worked reasonably well, it is recommended that in future, a second light source be placed on top of the test section so as to eliminate the dark area caused by the frame member that runs along the bottom of the test section. Figure 4.13(b) shows an example of this dark area.

To reduce the number of times that the water in the tunnel had to be changed, clear fluid was used for injection during the force-measurement tests. This was 
accomplished by connecting two reservoirs in parallel to the forebody nozzles as shown in figure 3.7. Flow meters were placed in the nozzle supply lines so that the rate of fluid injection can be measured. Injection fluid was switched between port and starboard nozzles by a three way, two position brass solenoid spool valve. Control of the solenoid valve was accomplished by a frequency control unit with an operating range between 0.011 to $0.51 \mathrm{~Hz}$. As well, the frequency control unit had a manual override feature that allowed the solenoid valve to be locked into either its energised (port nozzle injection) or de-energised (starboard nozzle injection) state.

As mentioned earlier, each pair of strain gauges mounted opposite to one another were wired to form one half of a Wheatstone bridge. The remaining resistances required to form two full Wheatstone bridge circuits were supplied internally by a pair of strain indicators thus forming the two strain gauge bridge stations used for force measurement [Perry and Lissner, 1955]. Outputs of the strain indicators were recorded by a two channel stripchan recorder whose response time was about $0.5 \mathrm{sec}$. Figure 3.8 shows some of the components used in the test setup. A complete list of the test equipment used is provided in appendix $A$.

Initially, a stripchan recorder was used because all of the available data acquisition systems were tied up in other projects. However, as more literature was researched, it became evident that due to the random fluctuations and sometimes oscillatory nature of the side forces being measured, the use of an analog device such as 
a stripchart recorder rather than a digital computer to record these signals had certain advantages. For example, the ability to graphically inspect the variation in side forces with angles of attack and sideslip as they were being measured provided for instant verification in repeatability.

\subsection{Preparations for Testing}

This section discusses the steps taken to prepare the model for testing and addresses the problems encountered during this process.

\subsubsection{Model Alignment}

After placing the model assembly into the water tunnel, the pitch and yaw angle indicators had to be aligned. Measurement of the test section with a level and a combination square revealed that the top and bottom test section walls were level and that the panels opposite one another were in fact parallel. Alignment of the pitch angle indicator was accomplished by placing a level on the afterbody of the model and adjusting the indicator accordingly. Aligning the yaw angle indicator was done by centring the model between the test section walls. Yaw alignment was done before each test run that involved a change in yaw angle. Circumferential alignment of the nozzles was accomplished by carefully mounting and levelling the entire model assembly on a machinist's flat. A height gauge was then used to measure the height of each nozzle from the surface of the machinist's flat. The forebody was then rotated until both nozzles were 
at the same level. These alignment procedures resulted in an accuracy of pitch and yaw angles of about $\pm 2^{\circ}$ and an accuracy in roll angle of about $\pm 0.5^{\circ}$.

\subsubsection{Troubleshooting}

As mentioned earlier, the original intent was to cunduct experiments, including force measurement, at relatively low free stream velocities. Consequently, the material selected for the sting had to have a small Young's modulus of elasticity so that the low aerodynamic forces generated at these slow speeds could be detected by the strain gauge balances. The sting material also had to be corrosion resistant and the strain gauges and leadwires had to be protected from moisture. To meet all of these specifications, a sting was manufactured from PVC. Once the strain gauges were mounted and wired, the sting was coated with polyurethane and encased in silicone sealing compound.

The first time the strain gauges were powered with this sting configuration, the output fluctuated wildly. In some cases the sting appeared to act as an antenna picking up stray electromagnetic signals including the peculiar square wave-type signal shown in figure 3.9. These fluctuations got even worse when the sting was immersed in water. Thinking that the metallic water tunnel itself was compounding the problem, the entire test section was shielded by coiling a grounded wire around it. This had no effect at all. After considerable analysis and testing, it was determined that these symptoms were actually being caused by a combination of three different problems. The first was a sensitivity to water. Coating the sting with polyurethane and silicone proved to be 
insufficient waterproofing. Evidently, the chlorine in the water was affocting the integrity of the seal. Also, the vinyl coated leadwires were being affected by the chlorine and began to absorb moisture. Secondly it was found that PVC exhibited visco-elastic behaviour which prevented acceptable repeatability. Clearly, a new material was required for the sting. The third, and last, problem was that of the stray electromagnetic signals that appear from time to time in the lab. The strain gauges and their leadwires appeared to be acting as antennae, occasionally picking up these electromagnetic signals.

Aluminum was chosen as the new material for the sting. To compensate for the higher Young's modulus, the cross sections where the strain gauges were mounted were machined down to a $1 / 16$ in $(1.6 \mathrm{~mm})$ thickness. To protect the electronics from electromagnetic interference, the strain gauges were wired using shielded cables and the sting itself was grounded. A schematic of the wiring configuration is shown in figure 3.10. Lastly, adequate waterproofing was accomplished by applying a series of protective coatings as follows: Referring to figure 3.11(a), after mounting the strain gauges onto the sting, they were coated with polyurethane as before. The section of the sting containing the strain gauges was then coated with a microcrystalline wax and wrapped with teflon tape. Finally, the entire area was encased with two coats of a polysulphide rubber compound [Dally and Riley, 1991]. For the leadwires, as shown in figure 3.11(b), teflon coated wires were considered as an alternative to vinyl, however, in order for the protective coatings used on the sting to adhere, the teflon would have to be etched. Etching teflon is a hazardous process and pre-etched teflon wiring was found to be too 
expensive. Therefore, it was decided to use vinyl conted wires inserted in plastic tubing and to coat the exposed areas with a nitrile rubber compound. The supplier and brand names for all of these materials are listed in appendix $\mathbf{A}$.

Referring back to figure 3.6, another problem that surfaced as the initial series of experiments progressed was that the drag created by the nozzle injection supply lines was exerting forces on the sting thercby biasing the strain gauge outputs. It was determined that the strain gauge leadwires were not contributing to this problem because they were secured to the sting at a point aft of the strain gauges behind where the sting was fastened to the pitch control linkage. Consequently, the blowing supply lines were secured in a similar fashion. The resulting contribution of the supply lines to the forces detected by the strain gauges was negligible.

\subsubsection{Calibration}

Mounted on the water tunnel control panel is an RPM meter that is supposed to indicate the impeller shaft speed. At the start of this project, this meter was not operational and its accuracy was in question. Therefore, in order to determine the velocity of the free stream fluid during testing, a calibration curve relating free stream velocity to impeller shaft speed was obtained. This was accomplished by timing how long it took dye to travel a fixed distance along the length of the test section at various impeller shaft speeds. Shaft speeds were determined by timing how long it took for the shaft to complete several full rotations. The resulting linear relationship is shown in 
figure 3.12.

Calibration of the sting balances was accomplished by hanging weights at a known distance away from the strain gauges and measuring the output of the Wheatstone bridges. The Young's modulus of elasticity for the sting ass_mbly was calculated for each data point and was found to be repeatable to within $\pm 6 \%$. Calibration curves relating output voltage to appiied force for both the aft and forward mounted strain gauge bridges were then obtained. The resulting linear relatuons, shown in figure 3.13. uere used to relate the voltage output measured by the stripchan recorder to the strain applied on the sting. This strain was, in turn, used to calculate the side forces applied on the model as well as their respective centres of pressure by the equations whose derivations are outlined in appendix C. Yaw moments were then determined using these side forces and centres of pressure.

\subsubsection{Nondimensionalising}

Originally, the reference area and span considered for nondimensionalising the coefficients were the projected area of the forebody and the base diameter of the model respectively. It was later decided to use the cross sectional area of the model as the reference area. The rationale behind this decision was to keep the reference area and span independent of nosecone length dimensions. That way, if a different size or shape nosecone were to be used for similar experiments in the future, the resulting coefficients may be compared with those obtained in the present research without any additional 
calculations.

\subsection{Experimental Procedures}

This section contains a brief outline describing the experimental procedures followed during the course of these experiments. Before each time the test section was filled, alignment of the model assembly yaw and pitch angle indicators was verified. After filling the test section with water, the model was set to the $\alpha=10^{\circ}$ and $\beta=0^{\circ}$ position and the free stream velocity was adjusted. The desired level of momentum blowing and frequency of oscillation were then adjusted. Frequency of oscillation, level of momentum blowing and free stream velocity were kept constant during each complete test. A typical test run started at zero sideslip and consisted of an increasing pitch angle sweep from $\alpha=10$ to $70^{\circ}$ in increments of $10^{\circ}$, then adjusting the sideslip angle to $\beta=-$ $10^{\circ}$ and sweeping the model in pitch back from $\alpha=70$ to $10^{\circ}$, again in increments of $10^{\circ}$. The sideslip angle was then adjusted to $\beta=-20^{\circ}$ and once again an increasing pitch angle sweep was performed. This procedure was repeated for $\beta=+10$ and $+20^{\circ}$. At each interval in pitch, either force measurement or flow visualisation data was collected for about one to two minutes. At the higher angles of attack, where fluctuations in the flowfield were larger. this time interval was sometimes as long as five minutes.

For a force measurement test run, the free stream velocity was set to between 12 and $14 \mathrm{in} / \mathrm{sec}(30.5$ and $35.6 \mathrm{~cm} / \mathrm{sec})$. Typical voltage outputs at these speeds for the higher angles of attack. as measured by the stripchart recorder, were about 0.5 Volts for 
the aft strain gauge bridge station and 0.3 Volts for the forward strain satuge bridge station.

Flow visualisation and video taping were performed at a free stream velocity between 2 and $3 \mathrm{in} / \mathrm{sec}(5.08$ and $7.62 \mathrm{~cm} / \mathrm{sec})$. Although voltage outputs from the strain gauge bridges were not generally measured during these tests, on the occasions in which measurements were made, the voltages were typically one tenth those measured at the higher speeds. Video taping and photographing of the flowfield were done by positioning the cameras on a tripod in front of the starboard side of the model. Lighting came from behind the mylar grid positioned on the port side of the model.

Considering the accuracy of the calibration tests (recall that the measured Young's modulus of elasticity was accurate to within $\pm 6 \%$ ), as well as the limitations of estimating mean and fluctuation voltage values from the stripchart recorder, the estimated accuracy for the side force and yaw moment coefficients presented in the next chapter are estimated to be within $\pm 10 \%$ for the higher speed tests. 


\section{$4 \quad$ Results and Discussions}

\subsection{Adopted Sign Convention}

With the $X$ and $Y$ coordinate axes shown in figure 4.1, applicatint, of the righthand rule results in the $\mathrm{Z}$ coordinate axis being positive upwards. If the modei that is superimposed on the axes is being viewed from above, then a force in the positive $Y$ direction must be a force to starboard. By the same rationale, a force to port would be one th.at is in the negative $\mathrm{Y}$ direction. Applying the right-hand screw rule about the positive $\mathrm{Z}$ axis yields the dircction of a positive yaw moment. Keeping in mind that the moments are being taken about the apex of the nosecone, one sees that the moment caused by a force to starboard is positive while that caused by a force to port is negative. Thus, forces and moments in the starboard direction will be taken as positive while forces and nıoments in the port direction will be assigned negative values. Also, yawing the model to starboard will be called positive sideslip (positive $\beta$ ) while negative sideslip will refer to the model yawing to port. Lastly, the angle of attack, $\alpha$, is positive when the model is in a nose-up attitude in the $X, Y, Z$ coordinate system of figure 4.1 .

\subsection{Clean-Forebody Flowfield}

This section discusses the flowfield around the forebody prior to the installation of injertion nozzies. The nosecone in this unmodified state will be referred to as the cleari-forebody. Figure 4.2 shows the side force and yaw moment coefficients measured on the clean-forebody at zero sideslip along with a sample of the stripchart recorder 
output collected during these tests. Because of the flurtuating behaviour of the lateral loads, $C_{y}$ and $C_{n}$ in figures 4.2(a) and (b) are presented as mean values together with maximum and minimum values to give the amplitude of the fluctuations. These values were extracted from the stripchart recordings as shown in figure $4.2(\mathrm{c})$.

For $10^{\circ} \leq \alpha \leq 30^{\circ}$ and $\beta=0^{\circ}$, the flowfield was symmetrical with no side forces or yaw moments present. At $\alpha=40^{\circ}$, there was a slight bias in the side force and yaw moment towards the starboard (positive) side as shown in figures $4.2(a)$ and (b). The fluctuations in side forces, although still very small, began to increase at this angle of attack, see tigure 4.2(c).

When the angle of attack was increased to $50^{\circ}$, significant asymmetries in the flowfield were detected. The resulting side force was in the starboard direction with moderate fluctuations as shown in figure 4.2(c). During the test runs that were conducted under similar conditions to establish repeatability, the flowfield at $\alpha=50^{\circ}$ displayed a side force that sometimes favoured the port direction. This phenomenon can be attributed to the bi-stable flow regime noted in chapter 2 although the angle of attack at which it occurred here was much lower than that observed by Malcolm (1991). That is, minor perturbations in the flowfield caused the vortex asymmetries to switch sides and become locked into a near mirror image of their previous orientation. Further observations to confirm this statement will be presented later in this chapter. 
For $x=60^{\circ}$, the flowfield became highly unstable with very large forces switching between port and starboard directions in a somewhat erratic manner, see figure 4.2(c). Flow visualisation revealed that the vortex produced on one side was building up strength and then shedding, then the vortex on the other side started to build up strength, eventually shedding and so on. It should be noted, however, that the time interval between vortices shedding was not constant.

As shown in figure 4.2(c), at $\alpha=70^{\prime \prime}$, large amplitude side force fluctuations about a mean near zero weri observed. Initially, it was thought that the flowfield became characteristic of a circular cylinder in cross thow as descrived in the literature [Malcolm, 1991] [Ng and Malcolm, 1991a] [Ng and Malcolm, 1991b]. However, the Strouhal number, $f d / V_{w}$ (where $f$ is the frequency of vortex shedding in $\mathbf{H z}, d$ is the base diameter and $V_{\infty}$ is the free stream velocity) [Simiu and Scanlan, 1986], for this phenomenon varied between 0.056 and 0.072 throughout the entire testing program. Degani and Zilliac (1988) quote 0.2 as a typical Strouhal number for an ogive-cylindertype model immersed in a free stream at an angle of altack of $70^{\circ}$ so although the cleanforebody flowfield at $\alpha=70^{\circ}$ does produce side force fluctuations with a very regular period, one cannot claim that it is purely representative of von Kármán vortex shedding phenomenon. There exists another important point for consideration at $\alpha=70^{\circ}$. Earlier, it was stated that the response time of the stripctart recorder was about $0.5 \mathrm{sec}$. Referring again to figure $4.2(\mathrm{c})$, as shown for $\alpha=70^{\circ}$, the distance between peaks varied from about 0.059 to $0.079 \mathrm{in}(1.5$ to $2.0 \mathrm{~mm})$. This indicates a time interval of between about 
0.6 and $0.8 \mathrm{sec}$ required for the asymmetric orientation of the flowfield to switch from port to starbaurd or vice versa. The fact that these time intervals are so close to the quoted response time of the stripchart recorder places the accuracy of the force measurements recorded at this angle of attack in question. The amplitude of the fluctuations plotted on the recorder may possibly be somewhat less than the actual amplitude being felt by the model. This potential restriction, however, does not have a particularly large impact on these experiments because, as will be shown later, the proposed method of oscillatory blowing has no effect on the flow field at $\alpha=70^{\prime \prime}$ (also sece appendix B).

Yawing the model resulted in a net side force in the same direction as the yaw angle. Fur example, yawing the model to starboard resulted in a force in the starboard direction and a force to port was noted when the model was yawed in the port direction. The magnitude of this force increased with increasing sideslip angle. These negative directional stability characteristics were not unlike those reported by Malcolm (1991) shown in figure 2.2(c). Figure 4.3 offers mean side force and yaw moment data for comparison. What is most evident when comparing figures $2.2(\mathrm{c})$ and $4.3(\mathrm{~b})$ is the large difference between the magnitudes of the yaw moment coefficients. This disparity is due to the fact that the moments presented by Malcolm were nondimensionalised by the reference area, S, and span, b, shown in figure 2.2(a) rather than using the base diameter, $d$, and corresponding cross sectional area, $(\pi / 4) d^{2}$, as was done for the present experiments. To compensate for this difference so that comparisons can be made more 
easily, Malcolm's values were converted to reflect the nondimensionalising convention practised in this study. These converted values are shown in brackets in figures 2.2 (b) and (c) as well as in figure 2.3 and were found to be of similar magnitude as those obtained from the present research.

Another simple test to validate the coefficients obtained from the present research is to check the ratio that relates the centre of pressure to the force and moment coefficients. This is expressed as follows:

$$
c_{n}-c_{n}\left(\frac{x_{c_{p}}}{d}\right)
$$

where: $\quad x_{c}$ is the location of the centre of pressure.

Selecting values from figure 4.3 , say at $\alpha=50^{\circ}$ and $\beta=0^{\circ}$, results in $C_{n} \approx 12.5$ and $C_{3}=3.5$ which yields a ratio of centre of pressure location to base diameter of about 3.6 . In other words, the centre of pressure is located about 3.6 diameters aft of the apex. Admittedly, this ratio is high but it still appears reasonable.

\subsection{Preliminary Nozzle Configuration}

The location and size of the first set of blowing nozzles was based on the recommendations made for optimum foruard hlowing in the literature [Malcolm and $\mathrm{Ng}$. 1991] [Malcolm, 1991] [Ng and Malcolm. 199 la] [Malcolm et al, 1989]. Longitudinally. the nozzles were located at $x^{\prime} d=1.0$ and radially at $\phi_{,}= \pm 135^{\circ}$, where $x$ is the distance 
between the nozzles and the apex of the nosecone, $d$ is the base diameter and $\phi_{1}$ is the radial location of the nozzles from the windward meridian. Testing revealed that this configuration performed unsatisfactorily, thus it shall oniy be discussed briefly.

During force measurement tests, the coefficient of blowing momentum, $\mathrm{C}_{\mu}$. required to alleviate the side forces that exist in the flowfield without blowing for the present configuration varied between 0.16 and 0.64 . Flow visualisation revealed that the nozzles in this location were too far downstream. Separation of the boundary layer had already taken place upstream and so the vorices were already well established by the time they travelled to the region influenced by the injection fluid. Thus the jet became entrained into the vortex flowfield without significantly altering the vortex trajectory. This action continued to take place until the blowing flow rate was increased enough that the jet stream could reach the area where boundary layer separation was taking place. At that point the magnitude of blowing momentum was too high to be practical. It was therefore concluded that the nozzles would be most effective if they were positioned longitudinally as close to the apex as physically possible.

Rolling of the nosecone such that $\phi_{j}= \pm 45^{\circ}$ only served to confirm what was concluded by Malcolm and Ng (1991a), Gittner and Chokani (1992), and Moskovitz et al (1988), namely that the inje ion of fluid from nozzles located on the windward sude of the forebody has no significant effect on the flowfield. Thus it uas iunded to keep the radial location of the nozzles at $\varphi_{1}= \pm 135^{\circ}$ from the windward meridian. 


\subsection{Optimised Nozzle Configuration}

Moving the nozzles as close to the apex as physically possible resulted in $x / d=0.25$. Also, in order to keep the size of the nozzles small relative to the nosecone, the nozzle opening diameter was reduced from $0.045 \mathrm{in}(1.14 \mathrm{~mm})$ to $0.020 \mathrm{in}(0.51 \mathrm{~mm})$. Preliminary test results using this configuration were promising, so it was decided to conduct the remainder of the experiments using this nozzle configuration.

\subsubsection{The Baseline Flowfield}

The flowfield around the forebody with the optimised nozzle configuration without blowing will be referred to as the baseline flowfield from now on. The object of this section is to define the baseline flowfield and to determine what effects the addition of injection nozzles had on the clean-forebody flowfield.

Comparing figures 4.3 and 4.4 , in general it can be concluded that the addition of nozzles to the nosecone in the optimised configuration has not had a significant effect except at $\alpha=50^{\circ}$ where, once again, there were inconsistencies. At first, it appeared as if the addition of nozzles caused the side forces to switch direction relative to the cleanforebody case. However, as with the clean-forebody flowfield, the side forces measured at this angle of attack occasionally switched directions.

\subsubsection{Baseline Flow Visualisation Test Results}

In order to perform a detailed analysis of the baseline flowfield, flow visualisation 
tests at zero sideslip were recorded using both video and still photograph cameras. The following is a description of the results obtained from these records.

For $10^{\circ} \leq \alpha \leq 30^{\circ}$, the flowfield was symmetrical as expected for such a low angle of attack range. Any vortices that did develop were visibly symmetrical and steady until reaching the model support sting. At $\alpha=40^{\circ}$, the vortices were forming symmetrically, that is, near the apex both port and starboard vortices were at the same distance away from the surface of the model. At a point farther downstream, the starboard vortex began to move away from the surface. Consequently, one would expect that a net side force to port would have developed. Instead, the force measurement tests, as shown in figure 4.5. showed a zero lateral load at $\alpha=40^{\circ}$ for zero sideslip. Closer scrutiny of the video tape later revealed that the point at which the starboard vortex started to move to a higher position was located a good distance aft of the end of the model afterbody, downstream of the strain gauge balance, and just upstream of where the sting connects to the pitch control linkage. This explains the zero side force readings because the balance cannot detect a force exerted in that area. It is doubtful that the aerodynamics of the model were responsible for this occurrence, quite possibly it was the interactions of the flowfield with the sting support and pitch control linkage that caused the starboard vortex to move to a higher position that far downstream.

At $\alpha=50^{\circ}$, as figure 4.6 shows, the starboard vortex is in a higher position than the port vortex. This indicates a net side force to port as confirmed by figure 4.5 . The 
port vortex burst location is about halfway down the length of the model and did not deviate significantly from that position. In contrast, the starboard vortex burst location is around three quarters down the length of the model but the burst location fluctuated upstream and downstream about that position. This motion corresponded to fluctuations on the stripchart recorder during the force measurement tests. During other test runs at $\alpha=50^{\circ}$, the vortices were both at the same distance away from the surface of the model but one was bursting at a point farther downstream than the other. The resulting asymmetries were such that the side forces and yaw moments generated were in the direction toward the side whose vortex burst farthest downstream.

The following describes the baseline flowfield at $\alpha=60$ and $70^{\circ}$ as observed during testing and later from the video. Unfortunately, it was not possible to adequately capture this motion using still photographs.

At $\alpha=60^{\circ}$, the flow pattem is unsteady, fluctuating in time. The description starts with the port vortex close to the surface and bursting just downstream of where the forebody joins the cylinder while the starboard vortex is in a higher position, bursting slightly downstream of where the port vortex burst is located. As time passes, the port vortex burst location stants to move upstream forcing the starboard vortex to move even farther away from the surface, however the starboard vortex burst stays in approximately the same location. The port burst continues to move upstream until it reaches the apex of the forebody then quickly moves back downstream and away from the surface of the 
modei. The starboard vortex reacts by moving closer to the surface while its burst location starts to move upstream. The port vortex burst continues to move downstream until it reaches a point just aft of where it originally started. At that time the starboard vortex burst, still moving upstream, reaches the apex of the forebody and then quickly starts to move back downstream taking on a higher position. The pon vortex burst then begins moving upstream as the port vortex moves closer to the surface until both vortices and their burst locations are back in their original positions. The cycle then repeats itself. It is interesting that the time period of this cycle tends to vary. Often, the vortices freeze into their positions for a fraction of a second.

The difference between baseline and clean-forebody flowfields at $\alpha=60^{\circ}$ is that the clean-forebody flowfield fluctuates about a net side force to port whereas the baseline flowfield fluctuates about a zero mean, see figures 4.2(a) and (b) and 4.5. Evidently, the nozzles have compensated for some kind of perturbation inherent in the clean-forebody that causes an asymmetric flowfield at this angle of attack.

The flow fluctuation cycle at $\alpha=70^{\circ}$, was similar to that outlined for $\alpha=60^{\circ}$ but much faster and more regular. Also, the vortex bursts occurred more upstream, about halfway along the length of the forebody. As was the case with the clean-forebody, the baseline flowfield displayed side force fluctuations with a very regular period. Thus the addition of nozzles on the forebody had no significant influence at this angle of attack A slight unsteadiness in the free stream fluid was revealed by the dye emerging from the 
upstream dye tube. After travelling some distance, but before reaching the model, the dye stream began to fluctuate and formed very small wave-like patterns. It is believed that this occurrence was caused by wall effects due to the fact that at $\alpha=70^{\circ}$, the forebody tip was relatively close to the test section wall.

\subsubsection{Baseline Flowfield Sensitivity to Reynolds Number}

To verify the consistency of the baseline flowfield at flow-visualisation speed $\left(R e_{d}=1500\right)$ with that at force-measurement speed $\left(R e_{d}=8000\right)$, tests were conducted in which force measurements were made at $\mathrm{Re}_{\mathrm{d}}=1500$. The ratio of Reynolds number between the two flow speeds was approximately 5 to 1, which is not a particularly large difference in terms of Reynolds number so similar results were expected. This assumption was verified by the results shown in figure 4.7. This shows side force and yaw moment behaviour of the baseline flowfield at the two flow speeds. The inconsistencies at $\alpha=40,50$ and $70^{\circ}$ may be attributed to the inaccuracy of the load measurements obtained from the stripchar recorder because the strain gauge balance outputs were very small at the lower flow speed. In fact, it would not be unreasonable to estimate the accuracy of these measurements to be within about $\pm 25 \%$.

\subsection{Blowing Effects on the Baseline Flowfield}

In order to determine how effective nozzle jet blowing was for the optimised configuration, a series of force measurement and flow visualisation tests were conducted. The results of these tests are outlined in the sections to follow. 


\subsubsection{Force Measurement Test Results}

The first series of tests consisted of force measurements where the minimum amount of blowing momentum required to cause a switch in the orientation of the asymmetries in the flowfield was noted. Then, the blowing momentum was increised until there were no further increments in lateral loading.

At zero sideslip, the first measurable deviations from the baseline flowfield occurred at $\alpha=20^{\circ}$ for $C_{k}=0.001$. These deviations, measured as lateral loads, increased with increasing blowing momentum. The amount of lateral force measured for a given coefficient of blowing momentum, $\mathrm{C}_{\mu}$, increased with increasing angle of attack. Positive blowing consistently resulted in a negative side force, that is blowing from the starboard nozzle generated a side force so port and vice versa. Figure 4.8 is an excellent example of the stabilising effect that blowing has on the fluctuations that occur naturally in the baseline flowfield. Recall that Malcolm (1991), observed the same stabilising effects. It should be noted that in figure 4.8 , as with all other samples of stripchart recorder outputs to follow, the aft bridge station ouiput lags that of the forward station by about 0.28 in $(7 \mathrm{~mm})$. This lag does not represent a phase shift between the response of the two strain gauge bridge stations, rather, it is caused by the plotting pens being mounted on the stripchart recorder with an offset thereby enabling them to travel across the full width of the chart paner without interfering with one another.

At $\alpha=60^{\circ}$, the initiation of blowing greatly reduced the fluctuations observed in 
the baseline flowfield as shown in figure 4.9. As well, the side forces were changed from a zero mean to either a positive or negative value dependent upon the direction of blowing as noted earlier. For $c=70^{\circ}$, blowing momentum coefficients as high as 0.1 failed to have any effect on the flowfield at all. The same general observations were made under non-zero sideslip conditions with blowing generating variations about a nonzero mean side force.

Figures 4.10(a) and (b) show the variation of mean side force and yaw moment generated with varying blowing coefficient at $\alpha=50^{\circ}$ and $\beta=0^{\circ}$. Figure $4.10(\mathrm{c})$ shows yaw moments obtained by Malcolm and $\mathrm{Ng}$ (1991) for the various nozzle configurations that they used. The additional scales shown in brackets in figure 4.10(c) are the values obtained by Malcolm and Ng's experiments but nondimensionalised by the base diameter of their model, in this case a $6 \%$ scale of the F-18 fighter aircraft. This was done in order to make their values comparable to those obtained during the present experiments. Conforming to the established sign convention, starboard blowing is assigned a positive value while a negative value is given to blowing from the port nozzle. Comparing the yaw moments in figure 4.10(b) with those of the forward blowing jet in figure 4.10(c), it can be seen that the responses of the flowfields are similar. In both cases, blowing generates a net yaw moment away from the blowing side. Also, there exists a limit beyond which increasing the blowing rate fails to increase the generated yaw moment. Of interest to note is the blowing effectiveness. Very little input is required to obtain very high output results. In fact, during a comparison between various yaw control 
methods, Malcolm (1991) determined that yaw control by forebody vortex manipulation required a mass flow rate approximately 36.5 times less than yaw control by directional jet thrusters positioned near the forebody apex.

For the remainder of this study, only the minimum coefficient of blowing momentum required to switch the orientation of the baseline flowfield is of interest. This is so because it is induced oscillations of the lateral loads that are of interest. Keeping this objective in mind, from the results of figure $4.10(a)$ and (b), it was decided to use $0.0011 \leq\left|C_{\mu}\right| \leq 0.0015$ for the remainder of this study. Although smaller blowing rates produced significant side forces at $\alpha=50^{\circ}$, they proved to have virtually no measurable effect on the baseline flowfield at higher angles of attack.

\subsubsection{Flow Visualisation Test Results}

As with baseline flow visualisation, nozzle injection tests conducted at zero sideslip were recorded with both video and still photograph cameras. A stream of red dye was injected into the flowfield by a dye tube located upstream of the model and the fluid emerging from the injection nozzles was coloured blue. Nozzle blowing for $10^{\circ} \leq \alpha \leq 40^{\circ}$ induced asymmetries into the ncrmally symmetrical flowfield. Blowing from the starboard nozzle caused the starboard vortex to move to a higher position thus a net side force to port was generated. Port nozzle blowing resulted in the pon vortex moving away from the surface thereby creating a net side force to starboard. 
At $\alpha=50^{\circ}$, as shown in figure 4.11, starboard tlowing causes the high starboard vortex that was characteristic of the baseline flowfield to take on a still higher position. Close inspection of the nozzle exit region reveals the stagnation zone that forces the vortex to lift away from the surface. Also evident from figure 4.11 is that during injection, the energetic fluid from the nozzle (blue dye) seems to end up inside the vortex core. This appears to allow the vortex on the blowing side to travel much farther downstream before bi ing than it did withou: olowing. Since, however, this vortex is already occupying the higher position, the existing asymmetry is enhanced. Port blowing, shown in figure 4.12 , reverses the asymmetric orientation of the baseline flowfield. forcing the same phenomenon just ciescribed for starboard blowing to occur on the port side. In both cases, the vortex opposite the blowing side moves closer to the surface of the model while its burst location moves slightly upstream.

The behaviour of the vortices at $\alpha=60^{\circ}$ when blowing is switched on is similar to that described for the baseline flowfield at $\alpha=60^{\circ}$; however the vortex burst locations no longer move upstream all the way to the apex, they now move upstream only as far as approximately halfway along the length of the forebody. Also, with blowing, the flowfield stabilises and the side force directions are no longer prone to random switching. Therefore the vortex on the blowing side stays in the higher position thus the side forces are always in the direction opposite the blowing side. Blowing does steady the vortices and allows for significant modifications in their trajectories but fluctuations are still very large. Fluctuations now occur with more regular periodicity, the vortices no longer 
temporarily freeze into their positions as often as they did without blowing.

Nozzle blowing at $x=70^{\circ}$ had no effect on the flowfield at all. Basically. the oscillatory mechanism characteristic of this angle of attack became more visible with the addition of dye from the blowing nozzles. Figure 4.13 shows this mechanism in action for starboard blowing with the burst location at its farthest downstream point shown in figures 4.13(a) and (b). When the vortex burst location moved to its farthest upstream point, near the apex, some of the injection fluid was observed getting entrained into the vortex on the copposite side, see figures $4.13(c)$ and (d).

\subsubsection{The Bi-Stable Regime}

Earlier it was noted that the asymmetries in the baseline flowfield at $\alpha=50^{\circ}$ were not always favouring the same side. This phenomenon was attributed to the flow being in the bi-stable regime as described in chapter 2 . It was stated that by inducing a perturbation, such as nozzle jet blowing, into the flowfield while in the bi-stable regime, the orientation of the asymmetries can be switched to form a near mirror image of their previous state. Furthermore, if the perturbation was removed, the asymmetries stayed in their new orientation. While studying the effects of nozzle jet blowing at $\alpha=50^{\prime \prime}$, the mechanism just described was observed. As figure 4.14 shows, switching the blowing from port to starboard and back to the port nozzle, the side force direction switched from starboard to port and back tc starboard as expected. However, when the blowing was switched off, rather than reverting back to the original state measured before the 
initiation of blowing, the asymmetric flowfield remained at the configuration associated with the last blowing rate. This phenomenon was unicue to the $\alpha=50^{\circ}$ region and it persisted during non-zero sideslip tests. It was not observed to occur at any other angle of attack.

Malcolm (1991) defined the bi-stable flow regime as occurring between $60^{\circ} \leq \alpha \leq 65^{\circ}$. For the model used in the present discussion, this region was found to cccur around $\alpha=50^{\circ}$. The difference may be attributed to differences in nose geometry. For example, the slenderness ratio of Malcolm's model, shown in figure 2.2(a), was $l / d \approx 4$ whereas for the model used here it was $\ell / d=2.25$.

\subsubsection{Individual Port and Starboard Blowing}

Figure 4.15 shous the side forces and yaw moments generated for individual port and starboard blowing (ie: no oscillation of blowing) with $\left|\mathrm{C}_{\mu}\right| \approx 0.0013$, the minimum blowing rate required to switch the orientation of the vortices for the full range of angle of altack tested. Data is presented as mean values with their respective fluctuations for $\beta=0,-10$, and $-20^{\circ}$.

As shown in figure 4.15(a), the side forces and yaw moments generated by port and starboard blowing were approximately symmetrical about zero. For cases with sideslip. $\beta=-10$ and $-20 \%$, see figures 4.15 (b) and (c) respectively. blowing had a similar efiect as in zero sideslip except that the curves now varied about a negative mean whose 
magnitude was dependant upon the degree of sideslip. Also, the symmetry in the shapes of the curves decreased with increasing sideslip angle. Similar observations were made for $\beta=10$ and $20^{\circ}$; the behaviour of the flowfield appeared to be virtually symmetrical to the $\beta=-10$ and $-20^{\circ}$ cases in shapes and trends.

\subsection{Maximum Allowable Frequency of Oscillatory Blowing}

With the ability of the blowing jeis to alter the asymmetries in the baseline flowfield well established, the next step was to investigate behaviour with relatively rapid back and forth switching of the blowing from one nozzle to the other, referred to as oscillatory blowing. This was accomplished by setting $\left|\mathrm{C}_{\mu}\right|=0.0013$, as before, and then oscillating the injection fluid back and forth between port and starboard nozzles. Two limiting factors had to be considered when determining the maximum frequency at which these oscillations should take place. First, the frequency had to be low enough to allow for aerodynamic lag effects. That is, the altered vortex trajectories had to be allowid to reach their steady state positions. Second, the frequency had to be high enough that the vortices did not have time to stabilise in these positions. Both factors were addressed by the procedure outlined below. The frequency that met these conditions was termed the maximum allowable frequency of osciliatory blowing.

The strain gauge balance outputs for various low frequency inputs are shown in figure 4.16. From these stripchart recordings, the time required for the induced asymmetries to reach steady state was estimated to be approximately two seconds, thus 
the maximum allowable frequency would be around $0.25 \mathrm{~Hz}$. With this frequency dialled into the frequency control unit, the resulting stripchart output showed that the vortices did stabilise for a small amount of time, so the frequency was slowly increased until all indications of this stabilisation vanished. This occurred at a frequency of about $0.33 \mathrm{~Hz}$. To better appreciate frequencies from an aerodynamics perspective, they will be expressed in the form of reduced, or nondimensional, frequencies as follows:

$$
\bar{\omega}-\frac{2 \pi f d}{V_{-}}
$$

where: $\quad \bar{\omega}$ is the reduced circular frequency of oscillation and will be referred to simply as reduced frequency,

$f$ is the frequency of oscillation in $\mathrm{Hz}$,

$d$ is the base diameter of the model, and

$V_{\text {. }}$ is the free stream velocity.

Thus the reduced, maximum allowable, frenuency of oscillatory blowing is approximately 0.16 for this model configuration.

\subsection{Oscillatory Blowing Effects}

Force measurement tests were made at $\bar{\omega} \approx 0.16$ and at $\bar{\omega} \approx 0.08$ in order to establish the behaviour of the flowfield under oscillatory conditions. At each angle of attack. the stripchart recorder was left running for some time in order to allow the 
asymmetries to oscillate through several cycles. Figure 4.17 shows some samples of the stripchart recorder outputs for both frequencies. At the lower frequency, see figure 4.17(a), the asymmetries not only have time to reach their steady state values but also have enough time to linger and fluctuate about those positions as indicated by the multiple spikes at the end of each wave. At the higher frequency, see figure 4.17(b), the single spike at the end of each wave indicates that the asymmetries are forced to start switching orientation just as soon as they reach their maximum values. As postulated at the end of chapter 2, evidently the random fluctuations int:erent in the baseline flowfield did in fact become superimposed onto these spikes. The results, shown graphically in figures 4.18 and 4.19 , are presented as mean amplitudes caused by port and starboard blowing with their respective fluctuations, see figure 4.17. The frequencies of o:cillation extracted from the stripcharts were the same as those input by the frequency control unit at all angles of attack except at $\alpha=70^{\circ}$ where the Strouhal number remained unchanged from that of the baseline flowfield.

From the graphs in figures 4.18(a) and 4.19(a), there do not appear to be any significant differences in the reactions of the flowfield between the two frequencies at zero sideslip. However it must be remembered that, at the lower frequency, the asymmetries remained in fixed orientations for some time before being switched. This was not the case for the higher frequency where the vortex trajectories were never allowed to settle down into a fixed patiern. 
Yawing the model resulted in observations similar to those in the zero frequency case, as shown in figures $4.18(\mathrm{~b})$ and (c) and 4.19(b) and (c).

\subsection{Increased Frequency Testing}

One question that it was felt necessary to address was what would happen if the frequency of oscillation was increased to a value much higher than the maximum allowable. Aerodynamic lag effects are then significant. With $\alpha=50^{\circ}, \beta=0^{\circ}$, and $\bar{\omega} \approx 0.54$, it was found that the asymmetries, which always favoured the port side with blowing off, very quickly switched over to the starboard side once oscillatory blowing was initiated, see figure 4.20. When the force fluctuations stabilised and began oscillating about a constant non-zero mean force to starboard, it was noticed that the forces responded more quickly to port blowing than they did to starboard blowing. In fact, as

shown in figure 4.20 , it took nearly twice as much time for the flowfield to react to starboard blowing than it did to react to port blowing.

Since the flowtield was in the bi-stable regime, a test was done in which the asymmetries were first sw'tched such that they favoured the starboard side and then the ascillations were re-initiated. As figure 4.20 shows, the flowfield adopted the same pattern as that just described: that is, fluctuations stabilised with the mean force remaining in the starboard direction.

Two hypotheses were suggested to explain why the flowfield responds more 
quickly to blowing from the port nozzle than the starboard nozzle. First, the port nozzle, for some reason, could be delivering more momentum than the starboari nozzle. Second, the port nozzle may be in a slightly more optimum position or have a slightly better configuration than the starboard nozzle and is therefore more effective at altering the vortical flowfield.

To test the first of these hypotheses, water was allowed to pass through one nozzle and empty into a graduated cylinder for a fixed amount of time. The volume of water in the cylinder was then recorded and the test was repeated for the other nozzle. Then, using this information, the volumetric flowrate exiting from each nozzle was calculated. Although there are flown ieiers connected to each of the nozzle supply lines, the flowrate was calculated in this manner to siiminate tie possibility of inaccuracies in flowmeter readings. The flowrate exiting from: the starboard nozzle was calculated to be $0.0389 \mathrm{in}^{3} / \mathrm{sec}\left(638 \mathrm{~mm}^{3} / \mathrm{sec}\right)$ while that from the pon nozzle was $0.0 .388 \mathrm{in}^{1} / \mathrm{sec}$ $\left(636 \mathrm{~mm}^{3} / \mathrm{sec}\right)$. Clearly, there is virtually no difference in the momentum being delivered by the nozzles; thus the first hypothesis was disproved.

Another observation that came to light during this test was that the fluid jets did not flow out of the nozzles symmetrically. Rather, their paths were as shown in figure 4.21. The fact that the streams did not follow symmetrical trajectories supports the second hypothesis. It appears tha: the direction of flow from the port nozzle made it more effective in altering the vortical flowfield. This asymmetry in nozzle configuration 
is due to small manufacturing and alignment discrepancies.

Referring back to figure $4.17(\mathrm{~b})$, closer analysis reveals that the magnitudes of the peaks on the starboard side (positive) are slightly greater than those on the pori side (negative). Keeping in mind the response of the flowfield at higher frequencies that was just discussed, this minor asymmetry indicates that the maximum allowable reduced frequency of oscillatory blowing for this forebody configuration may in fact be slightly less than 0.16 .

\subsection{Aircraft Inertial Response}

The objective of creating a zero time-average side force and yaw moment by the rapid switching of forebody vortex asymmetry has been essentially realised. The remaining issue is whether or not the maximum allowable blowing-oscillation frequency determined earlier is high enough that a full size aircraft in a post-stall manoeuvre would not be subject to excessive lateral oscillations due to the fluctuating side forces and yaw moments associated with oscillatory forebody blowing. This would depend on the inertial response of the aircraft in question. The iollowing discussion uses the information provided in figure 4.22 and appendix $D$ for an analysis of the inertial response of an F18 fighter aircraft to such an oscillatory yawing moment.

To maintain dimensional similariiy with the test model, the nozzles required for an F-18 would have a diameter of $0.720 \mathrm{in}(18.3 \mathrm{~mm})$. These nozzles would be flush- 
mounted 9 in $(22.9 \mathrm{~cm})$ aft of the apex at a radial position of $\phi_{1}= \pm 135^{\circ}$ from the windward meridian.

Using information from figure $4.19\left(\right.$ a) at $\alpha=50^{\circ}$ and $\beta=0^{\circ}$ results in a coefficient of yaw moment about the centre of giavity of an F-18 of $C_{n}=27$. For the comparison made between various yaw control methods mentioned earlier, Malcolm (1991) used a free stream velocity of $330 \mathrm{f} / \mathrm{sec}(100 \mathrm{~m} / \mathrm{sec})$ at an altitude of $15000 \mathrm{ft}(4.6 \mathrm{~km})$. This resulted in a dynamic pressure of $75 \mathrm{psf}(3.59 \mathrm{kPa})$. Application of these values at a reduced frequency of $\bar{\omega} \approx 0.16$ indicates that the pilot in an F-18 at maximum takeoff weight would experience an oscillatory amplitude of about 0.28 in $(7.11 \mathrm{~mm})$ peak to peak. Similarly for an empty F-18, the pilot would experience oscillations of about 0.68 in $(17.27 \mathrm{~mm})$ peak to peak. The real-time frequency at the stated conditions would be about $3 \mathrm{~Hz}$.

Although this motion is hardly negligible, during such a post-stall manoeuvre the pilot would be subjected to other effects such as g-force and vibrations due to buffeting so testing under actual flight conditions is requiræd in order to determine if these added oscillations are acceptable. Also, it remains to be seen if improved accuracy in the positioning of the nozzles would reduce the favouritism of the flowfield toward one side at higher frequencies thereby permitting an increase in the maximum allowable frequency of oscillation. This would result in a reduction in the maximum yaw amplitude felt by the pilot. For example. if the reduced frequency could be doubled to 0.32 , then the 
oscillations felt by the pilot would be reduced by a factor of 4 resulting in an oscillatory amplitude of about $0.18 \mathrm{in}(4.57 \mathrm{~mm})$ peak to peak. 


\section{Conclusions}

\subsection{Water Tunnel}

A lot of servicing was performed on the water tunnel during the preliminary work for this study. The author's experience in achieving and maintaining smooth, reliable operation of the water tunnel was discussed in detail in chapter 3 ; a summary of procedures to be prastised while operating the water tunnel is provided in appendix $E$.

\subsection{Experiments with the Clean-Forebody}

At lower angles of attack $\left(10^{\circ} \leq \alpha \leq 40^{\prime \prime}\right)$. the clean-forebody flowfield behaved as described in the literature. The bi-stable flow regime occurred at $\alpha=50^{\circ}$ rather than the higher $\alpha=65^{\circ}$ angle of attack reported by Malcolm (1991). This difference is probably due to the differences in slenderness ratio between the forehody used during the present experiments $(f / d=2.25)$ and that used by Malcolm $(f / d=4)$. The clean-forebody flowfield at $\alpha=60^{\prime \prime}$ was characterised by large amplitude side-force fluctuations; flow visualisation revealed asymmetries switching between the port and starboard sides of the model at irregular intervals. At $\alpha=70^{\circ}$, the clean-forebody flowfield exhibited large sideforce fluctuations with a very regular period. The Strouhal number varied between 0.056 and 0.072 compared with 0.2 for a typical ogive-cylinder-type model at the same angle of attack. Thus the flowfield is not purely representative of von Kármán vortex shedding pheno..enon. Flow visualism... on $u-70$ showed an unsteadiness in the free stream

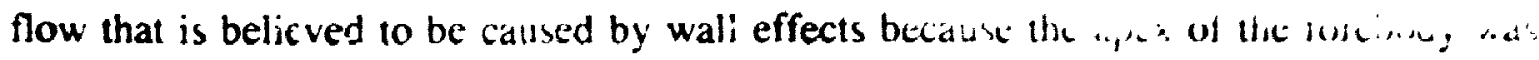


then relatively close to the bottom of the test section.

\subsection{Experiments with Nozzles}

The placement of nozzles in the position recommended in the literature for optimum forward blowing $\left(x / d=1.0, \phi_{j}= \pm 135^{\circ}\right)$ resulted in unsatisfactory performance. How visualisation showed that the nozzles were too far downstream; the vortices were already well established by the time they reached the nozzles. Therefore the blowing momentum had to be increased to impractical levels in order for the jet to produce significant effects on the vortices. As a result, the nozzles were moved as close to the apex as physically possible which was at $x / d=0.25$. Also, with the initial axial location of the nozzles at $x / d=1.0$, radial positioning at $\phi_{j}= \pm 45^{\circ}$ verified the observations made in the literature that nozzles placed on the uindward side of the model had no significant effects on the flowfield. Positioning smaller diameter nozzles at the new position; $x / d=0.25 . \phi_{1}= \pm 135^{\circ}$ resulted in good overall performance. Consequently the remainder of the experiments were conducted with this nozzle configuration.

The addition of nozzles at $x / d=0.25$ and $\phi_{1}= \pm 135^{\circ}$, which is termed the oriumised nozzle configuration, had no significant effects on the clean-forebody flowfield $\therefore \therefore$ tat at $a=50^{\circ}$ where inconsistencies in the flowfield can be attributed to the bi-stable flow regime. Vortex position asymmetries created side forces and yaw moments in the direction touard the vortex that was closest to the surface of the model. in agreement with the literature. On the occasions. at $\alpha=50^{\circ}$. when side forces and yaw moments were 
present with both vorices at the same distance away from the surface of the model, the side forces and yaw moments were found to be in the direction of the side whose vortex burst farthest downistream.

Blowing with the optimised configuration 'vas effective at angles of attack as low as $\alpha=20^{\circ}$ and sideslip angles up to $\beta= \pm 20^{\circ}$, the largest value investigated. The minimum coefficient of blowing momentum required to alter the flou field at angles of attack as high as $\alpha=60^{\prime \prime}$ was $C_{\mu}=0.0013$. Blowing had no effect on the thowtield at $\alpha=70^{\circ}$ regardless of the blowing magnitude. Starbodrd (positive) blowing always produced a side force to port (negative) and vise versa. Flow visualisation revealed that the vortex on the blowing side was forced away from the surface of the model and that the vortex on the opposite side reacted by moving to a closer position. for a constant magnitude of blowing momentum. the side forces and yau moments produced increased with increasing angle of attack up to $\alpha=60^{\circ}$. At any given angle of attack. the side force and yaw moment generated increased with increasing level of blowing momentum but there exists a limit beyond which further increases in blowing do not result in increased side force or yaw moment. Blowing effectiveness is high; that is very little blowing input is required to achieve very high side force and yaw moment outpul results. As was observed in the literature. blowing was found to reduce the fluctuations inherent in the baseline flowfield. Port and starboard blouing produced virtually symmetrical fevels of lateral loading about a mean near zero at zero sideslip. At non-zero sideslip. the side forces and yaw moments produced by port and starboard blowing varied almost 

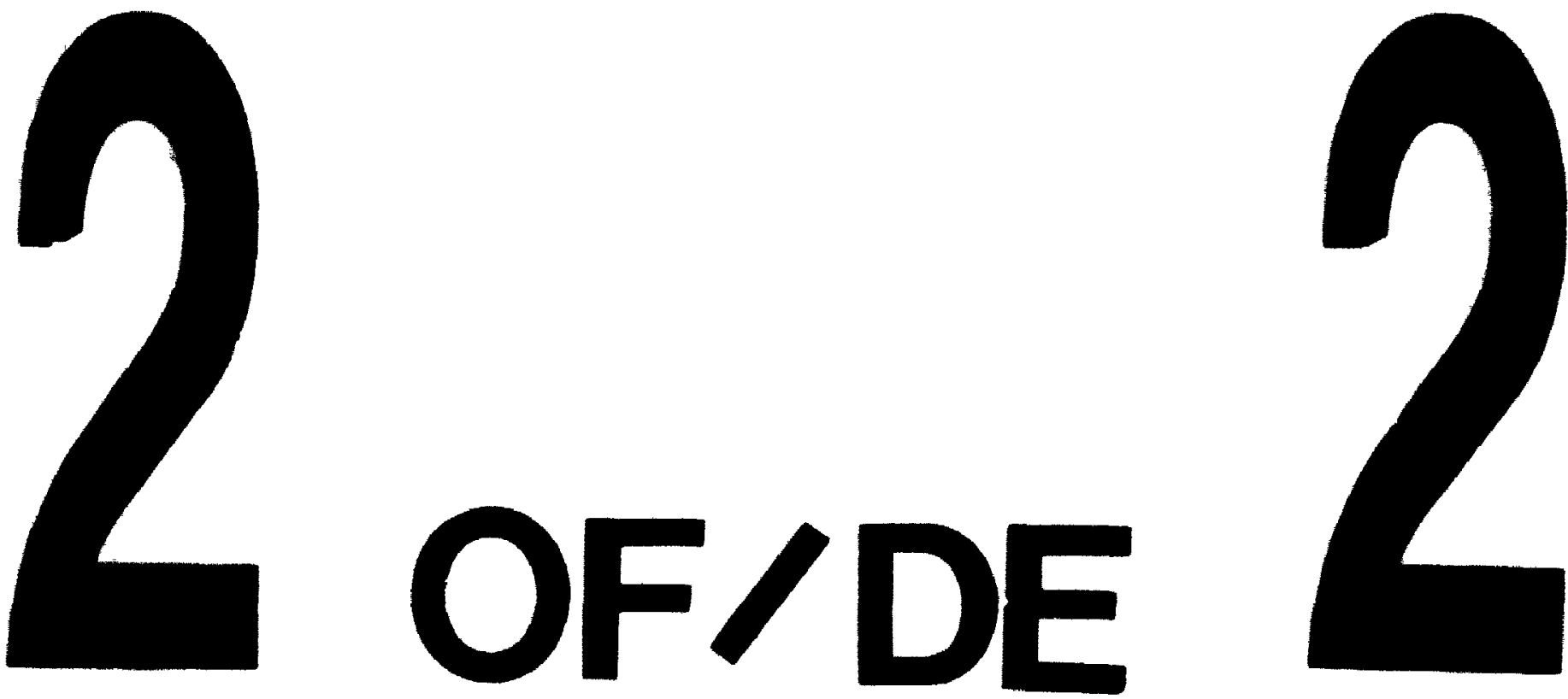

PM-1 $3: x 4$ PHOTOGAAPHIC MICROCOPY TARGET NBS 1010a ANSI/ISO \#2 EOUIVALENT

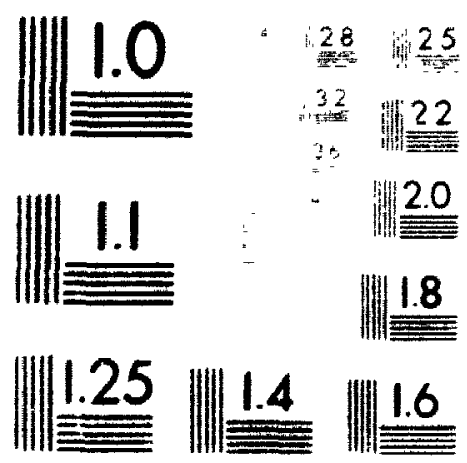


symmetrically about a non-zero mean whose magnitude depended upon sideslip angle. Symmotry in blowing effects decreased with increasing sideslip angle.

Oscillatory blowing did prodice relatively rapid switching of the flowfield asymmetries. The rapidity of su itching was restricted largely by aerodynamic lag effects. The maximum allowable reduced frequency of oscillatory blowing was found to be $\bar{\omega}=0.16$ for the optimised nozzle configuration. At $\bar{\omega}=0.16$, the random fluctuations inherent in the baseline flow field became superimposed onto the momentary lateral loads being generated by oscillatory blou ing. Lower reduced frequencies of oscillatory blowing were characterised by the asymmetries in the flowfield becoming steady for some time before being forced to switch direction. Oscillatory blowing had no effects at $\alpha=70^{\circ}$ : the Strouhal number of the spontaneous force and moment fluctuations remained unchanged from that of the clean-forebody flowfield.

When the reduced frequency of oscillatory blowing uas increased to a large enough value that aerodynamic lag effects became significant, the asymmetries in the flow field favoured the starboard side (ie: pon blowing). The fiowfield responded twice as quickly to port blowing as to starboard blowing. It is believed that this was due to slight differences between the two nozzles with the por nozzle apparently in a position which was more effective at altering the flowfield. Keeping this in mind. closer scrutiny of the response of the flowfield at $\bar{\omega} \approx 0.16$ revealed that the magnitudes of the side forces in response to port blowing were slightly larger than those caused by starboard 
blowing. This would suggest that some improvement in nozzle positioning is possible, enabling some increase in the maximum allowable frequency of oscillatory blowing to a value above $\bar{\omega} \approx 0.16$.

The estimated dynamic response of an F-18 fighter aircraft to oscillatory blowing at $\bar{\omega}=0.16$ suggests that amplitudes of oscillation would likely be felt by the pilot. However. it is believed that the potential for improved performance of this oscillatory blou ing "Ystem exists and that further investigation is merited. 


\section{Recommendations for Future Work}

\subsection{Water Tunnel}

There are certain measures that must be taken if successful operation of the water tunnel is to continue. First and foremost, the entire impeller drive shaft assembly both internal and external to the water tunnel must be overhauled. As well, the impeller itself is corroded and pitted and may require replacement in the near future, preferably with one that is made out of a non-corrosive material. Another general, but minor, repair is that of the leaks that exist all around the water tunnel circuit. Current practice is to temporarily stop these leaks with a silicone sealing compound thus a more permanent solution should be investigated. Lastly, if cavitation experiments are intended, then the high speed motor drive control is in need of servicing. Recall that the motor starts rotating at an unacceptably high rate just as soon as the control rheostat is touched.

\subsection{Test Equipment}

Model manipulation and data acquisition were performed manually during experimentation. On average, each test run as described in chapter 3 took about three hours. Manually reading of the side forces from the stripchart recordings and subsequer: careful entry of the data into a spread sheet for processing took an additional three hours per test. Automation of this entire process would considerably reduce the time required to obtain the final results from each test. This can be accomplished by connecting the strain gauge bridges to a data acquisition system with a high enough sampling rate stch 
that the fluctuations of the input signal would be accurately recorded. Impeller shaft speed and injection flow rates can be recorded in a similar fashion thereby fully automating the data acquisition process. A computer program can be used to actuate traverse gears for adjustment of the pitch and yaw angles of the model as well as to control the frequency of oscillatory blowing. Once a test run is completed, data reduction and graphing of final results can be performed automatically on site.

\subsection{Experiments}

The retically subjecting an F-18 fighter aircraft to the oscillatory mechanism investigated in this study resulted in the pilot experiencing oscillations with an amplitude up to about $0.68 \mathrm{in}(17.27 \mathrm{~mm})$ peak to peak. Testing under actual flight conditions is required in order to determine if these oscillations are negligible compared with the $\mathrm{g}$ force and buffeting vibrations that a pilot would feel during such a post-stall manoeuvre. The result calculated for the F-18 was only a rough estimate since it was obtained by taking some of the data collected in this study for a siender nosecone-afterbody model and applying it to an actual aircraft. Experiments in this study were conducted at very low Reynolds numbers $\left(\operatorname{Re}_{d}=8000\right.$ ). Further experimentation at more realistic Reynolds numbers constitutes an important future task. A $20 \times 30$ in $(51 \times 76 \mathrm{~cm})$ wind tunnel, for example, can accommodate a slender nosecone-afterbody model with a base diameter of about 3 in $(7.6 \mathrm{~cm})$ for variations in angle of attack up to $\alpha=75^{\circ}$ in the 30 in $(76 \mathrm{~cm})$ direction. With a free stream velocity of, say, $200 \mathrm{ft} / \mathrm{sec}(61 \mathrm{~m} / \mathrm{sec})$, the resulting Reynolds number based on body diameter would be about 0.28 million. Also, an 
analytical study of oscillatory blowing under actual fiight conditions could yield further insight into the applicability of this phenomenon.

It is believed that the favouritism of the flowfield toward the starboard side at high frequencies of oscillatory blowing was due to the blowing nozzles being slightly skewed as a result of manufacturing imperfections. More accurate positioning of the nozzles could result in the reduction of this favouritism on future generations of models thereby allowing the maximum frequency of oscillatory blowing to be increased. This would in turn reduce the resulting oscillatory amplitude that a pilot in an actual aircraft would feel. Another approach would be to compensate for the above mentioned favouritism by applying either different blowing rates for port and starboard nozzles or oscillatory blowing with an asymmetric time delay. These methods can also be used to investigate the possibility of generating non-zero time-averaged side forces and yaw moments for directional control. Furhermore, research is required into the placement, orientation, and exit geometry of the nozzles. For example, blowing effectiveness could. quite possibly. be improved by positioning the nozzles at a cant angle relative to the longitudinal axis of the model. 


\section{References}

Beer, F.P., Johnston, E.R., Mechanics for Engineers--Starics and Dynamics, third edition, McGraw-Hill, 1976.

Beer, F.P., Johnston, E.R., Mechanics of Materials, McGraw-Hill, 1981.

Comelius, K.C., Pandit, N., Osborn, R.F., Guyton, R.W., An Experimental Study of Pneumatic Vortex Flou Control on High Angle of Attack Forebody Model. AIAA-920018, January 1992.

Dally, J.W., Riley, W.F., Experimental Stress Analysis, third edition. McGraw-Hill, 1991, pp. 196-199.

Deganı. D.. Zliliac, G.G.. Experimental Study of Nonsteady Asymmetric Flow Around an Ogive-Cylinder at Incidence. AlAA-88-4330, August 1988.

Etkin, B., Dynamics of Flight: Srabiling and Control, second edition. Iohn Wiley and Sons. 1982, pp. 71-75.

Font, G., Tavella. D.. High-4lnha Aerodsnamic Control by Tangential Fuselage Blowing. AIAA-91-0620, January 199

Gerhart. P.M., Gross, R.J.. tididumentals of Fluid Mechanics, Addison-Wesley, 1985.

Gittner. N.M., Chokani. N.. The Effects of Nozle Geometry on Forebody Vonex Control Using Blowing, AIAA-92-2603-CP. June 1992.

Guyton, R.W., Maerki, G., X-29 Forebody Jet Blowing, AIAA-92-0017, January 1992.

Guyton, R.W., Osborn, R.F., LeMay, S.P., Forebody Vortex Control Aeromechanics, AGARD-CP-497. Paper Number 16, November 1991.

LeMay, S.P., Sewall, W.G. Henderson, J.F.. Forebody Vortex Flow Control on the FI6C Using Tangentiul Siot und Jet Nozle Blowing, AlAA-92-0019, January 1992.

Malcolm. G.N., Forebody Vortex Control, AGARD-R-776, Paper Number 6. April 1991.

Malcolm. G.N.. Ng. T.T. Aerodynamic Control of Fighter Aircrafi by Manipulation of Forebody Vortices, AGARD-CP-497, Paper Number 15, November 1991.

Malcolm, G.N., Ng, T.T., Lewis, L.C., Murri, D.G., Development of Non- 
Conventional Control Methods for High Angle of Attuck Flight Using Vortex Manipulation, AIAA-89-2192-CP, July-August 1989.

Merzkirch, W., Flow Visualization, second edition, Academic Press, 1987.

Moskovitz, C.A., Hall, R.M., DeJamette, F.R., Effects of Surface Perturbations on the Asymmetric Vonex Flow Over a Slender Body, AIAA-88-0483, January 1988.

Moskovitz, C.A., Hall, R.M., DeJamette, F.R., Experimental Investigarion of a New Device to Control the Asymmetric Flowfield on Forebodies at Large Angles of Attack. AIAA-90-0068, January 1990.

Murman, S.M., Rizk, Y.M., Cummings, R.M., Schiff, L.B., Computational Investigation of Slot Blowing For Fuselage Forebody Flow Control, AIAA-92-0020, January 1992.

Nakayama. Y.. Woods. W. A.. Clark. D.G.. Visualized Flon: Fluid Motion in Basic and Engineering Situations Revealed by Flow Visualization, Japan Society of Mechanical Engineers, Pergamon Press, 1988.

Ng, T.T., Malcolm, G.N., Aerodynamic Control Using Forebody Blowing and Suction, AlAA-91-0619, January 1991 la.

Ng, T.T., Malcolm, G.N., Aerod!namic Control Using Forebody Strakes. AIAA-910618. January 1991b.

Peake, D.J.. Ou'en. F.K., Johnson, D.A., Control of Forebody Vortex Orientation in Alleviate Side Forces, AIAA-80-0183, January 1980.

Perry, C.C.. Lissner, H.R., The Strain Guge Primer. McGraw-Hill. 1955. pp. 45-70.

Raymer, D.P., Aircrafi Design: A Conceptual Approach, AIAA, 1989, pp. 439-440.

Ross, A.J., Jeffries, E.B.. Edwards, G.F., Dynamic Wind Tunnel Tests on Control of Forebody Vonices with Suction, AGARD-CP-497, Paper Number 17. November 1991.

Simiu, E.. Scanlan, R.H., Wind Effects on Structures, an Introduction to Wind Engineering. second edition, John Wiley and Sons. 1986, pp. 133-140.

Thomson, W.T.. Theon of Vibration With Applications, second edition. Prentice-Hall, 1981, pp. 13-36.

White, F.M., Viscous Fluid Flow. McGraw-Hill. 1974. 
Figures 


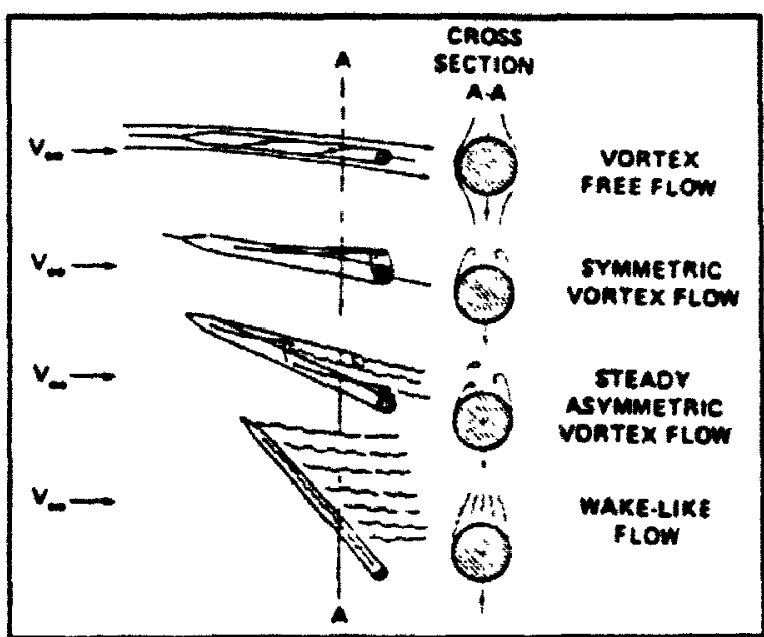

Figure 2.1 Flowfield description around an ogive-type forebody-cylinder model for ucreasing angle of attack. Reproduced from figure I of Malcolm (1991).

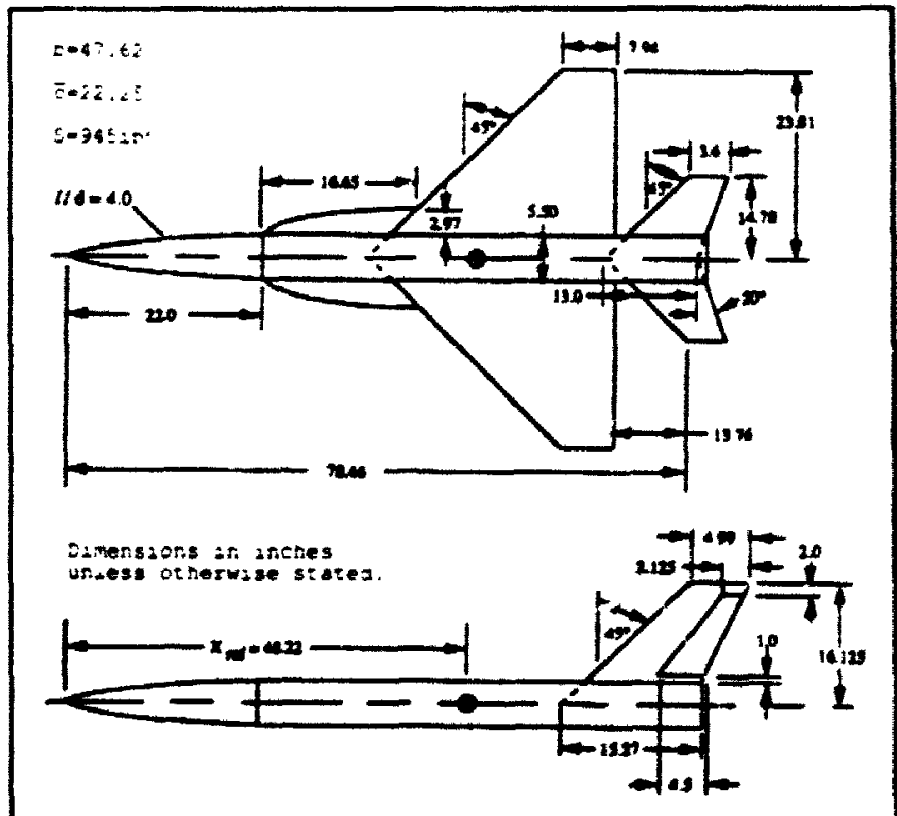

Figure 2.2(0) Schematic of generic fighter model. Reproduced from figure $I$ of Malcolm and $\mathrm{Ng}_{\mathrm{g}}(199 \mathrm{I})$. 


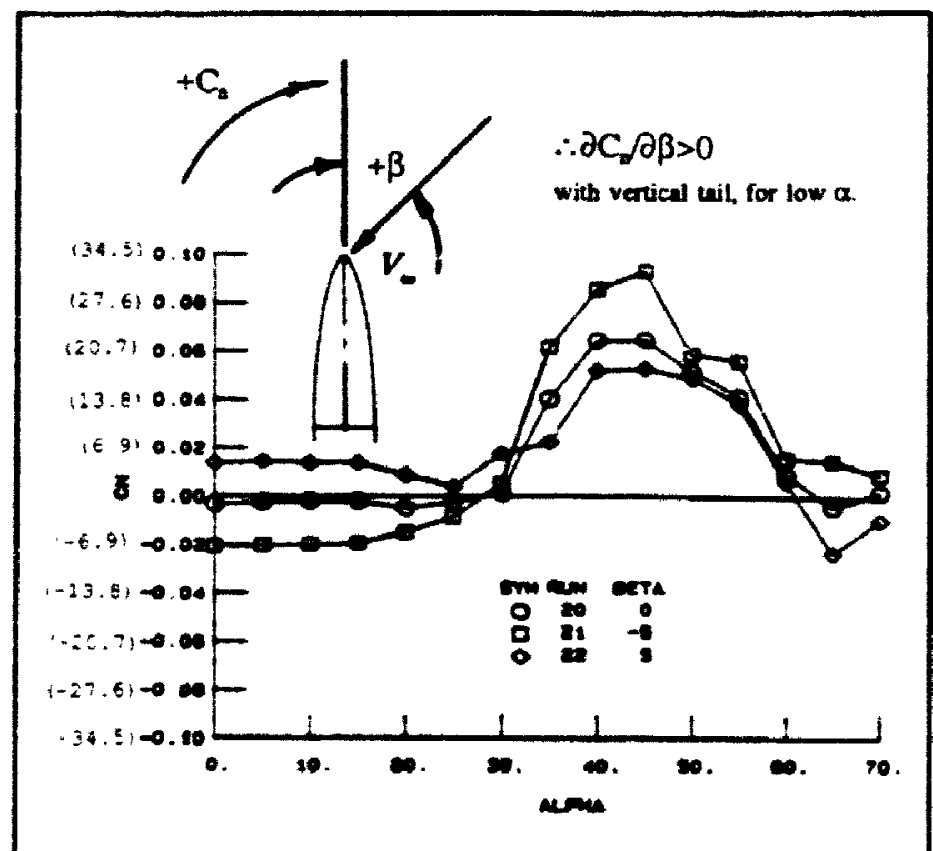

Figure 2.2(b) Yaw moment coefficient for generic fighter model. Reproduced from figure 16(a) of Malcolm (J991).

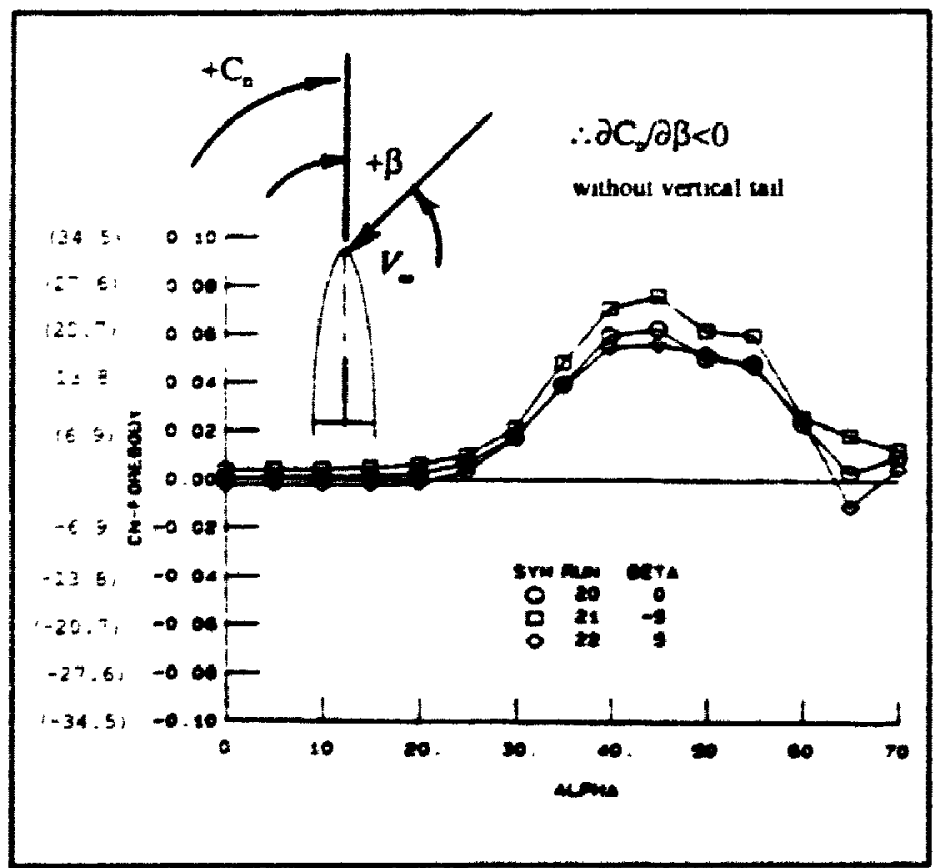

Foure 2.2(c) Forebody contribution to yaw moment coefficient of genesic fighter model. Reproduced from figure $16(0)$ of Malcolm (1991). 


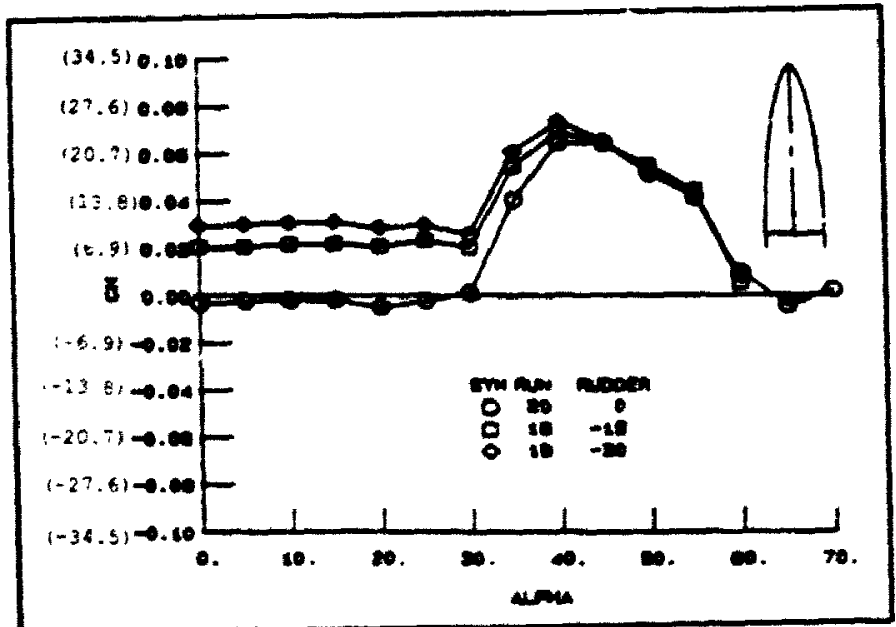

Figure 2.3 Yaw moment coefficient for various rudder deflections of generic fighter model. Reproduced from figure 17 of Malcolm (1991).

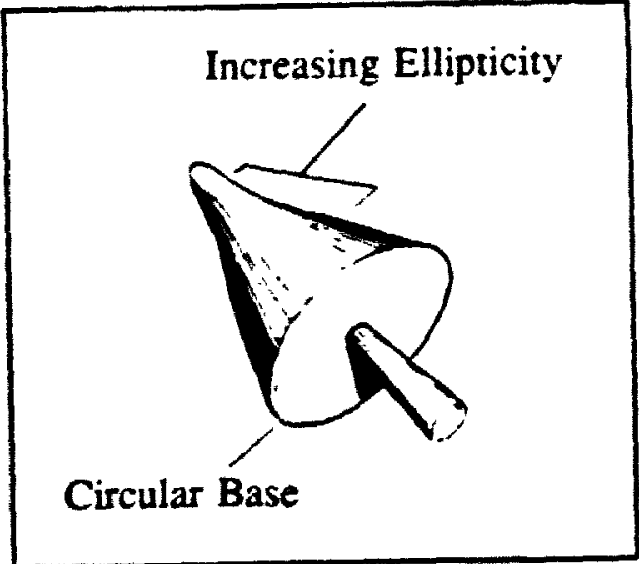

Figure 2.4 Rotatable elliptical tip schematic. Reproduced from figure 1 of Moskovitzer al (1990). 


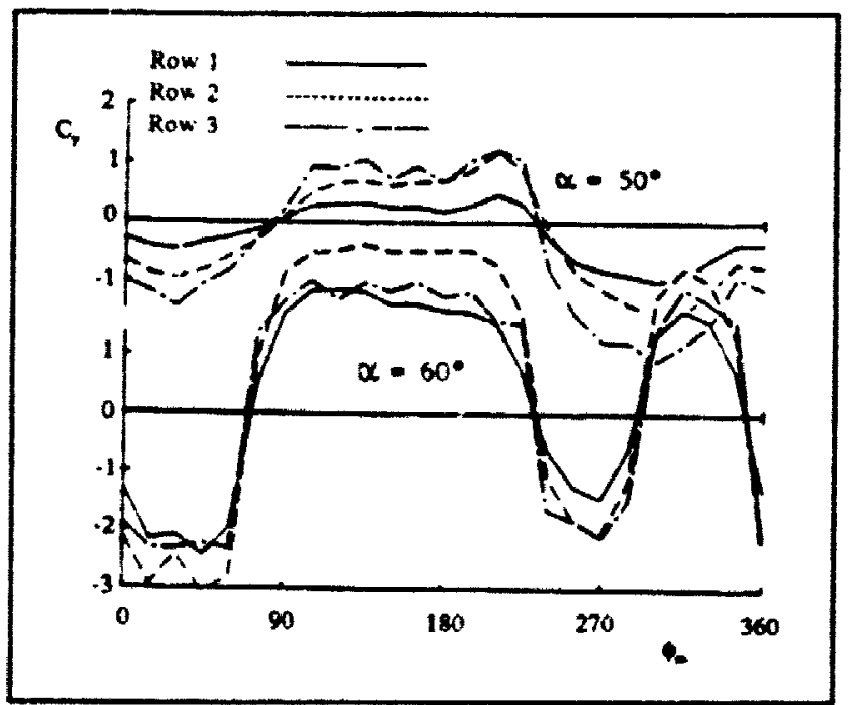

Figure 2.5(a) Side force coefficieat versus tip roll position for the sharp axisymmetric tup. Reproduced from figure 10 of Moskonta ef al (1990).

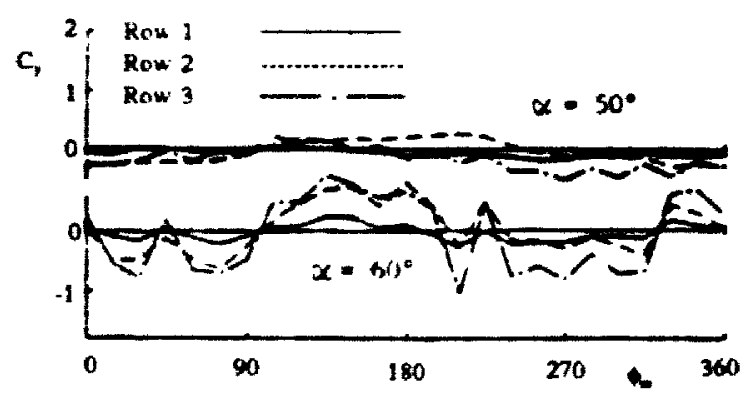

Figare 2.5(b) Side force versus tip roll position for the blunt axisymmetric tip. Repraduced from figure II of Moskovitz et al (1950). 


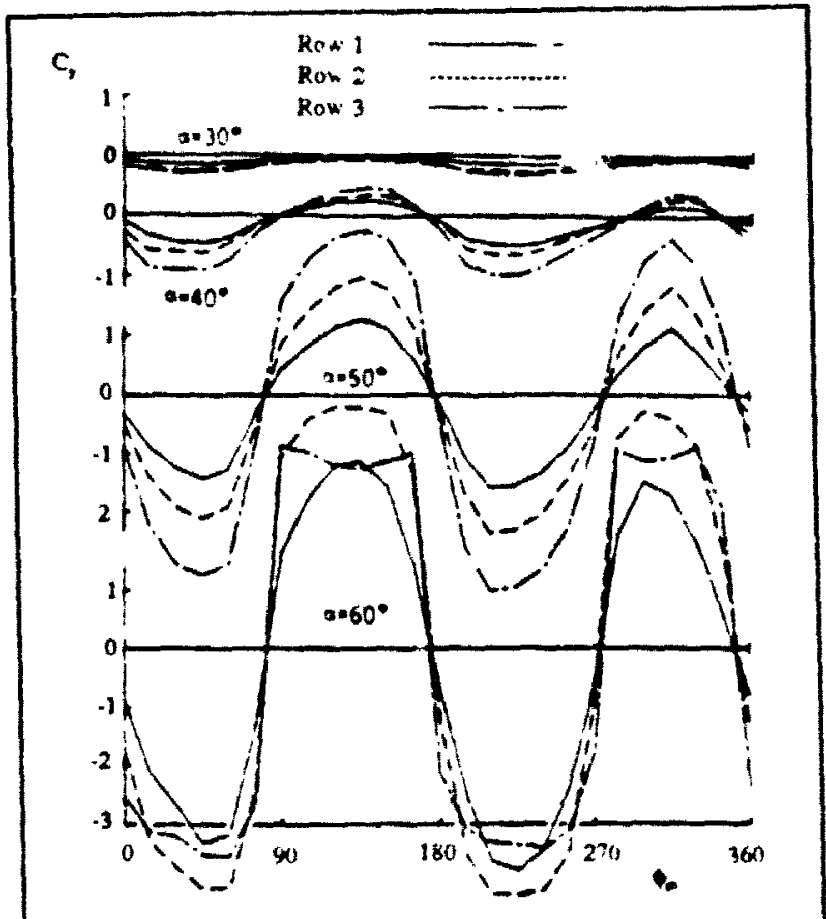

Figure 2.6(a) Side force versus tip roll position for elliptical up 01. Reproduced from figure 12 of Moskovite et al (196(1).

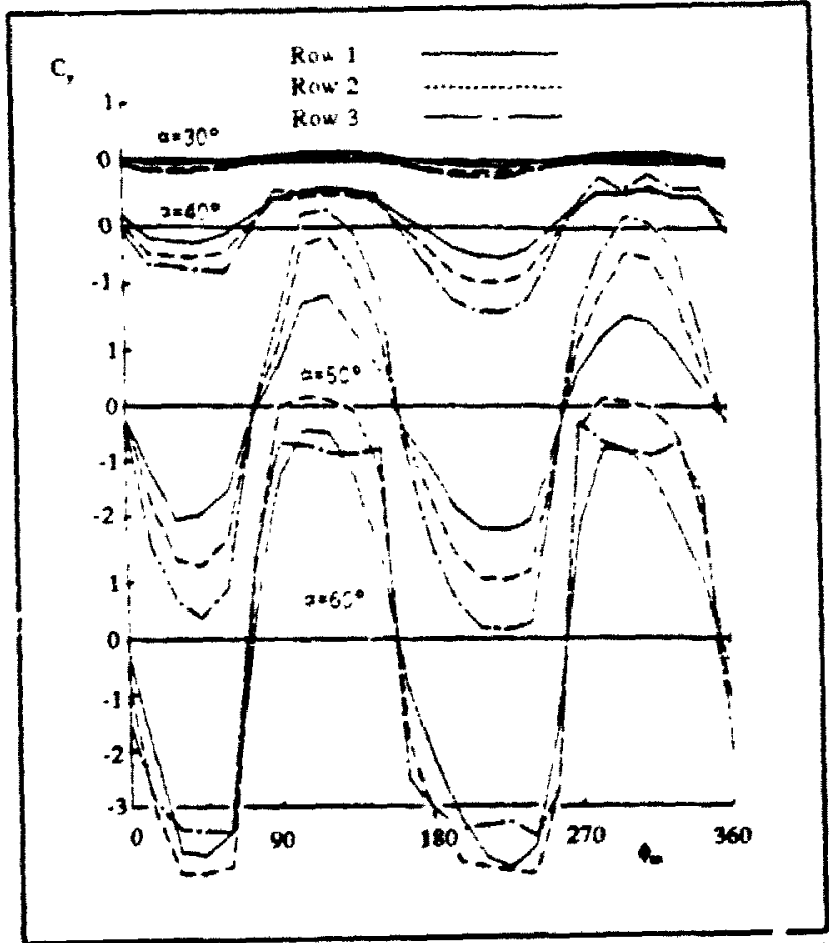

Figure 2.6(b) Side force versts tip roll position for ellir cal tip 02. Reproduced from figure 13 of Moskovirz et al (1 490$)$. 


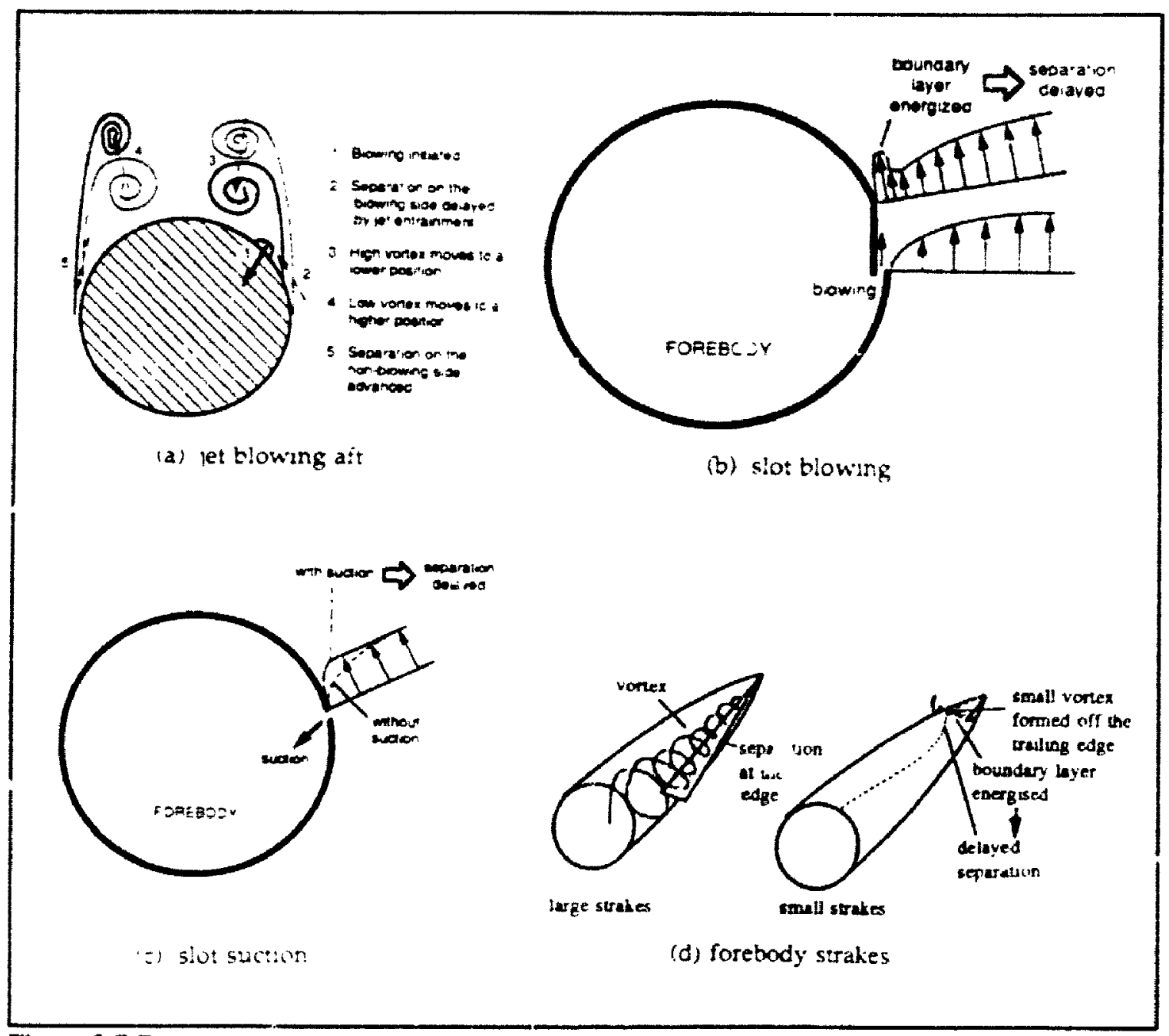

Figure 2.7 Fluid mechanics of various forebody vortex control methods. Reproduced from figure 16 of Malcolm and $\mathrm{Ng}$ (/S9l). 


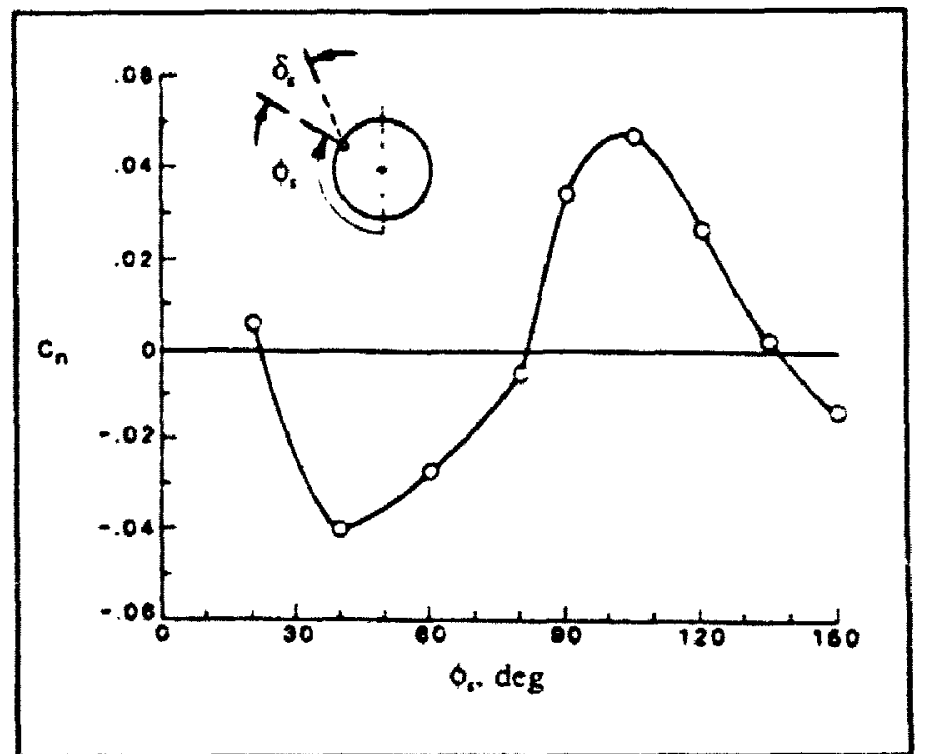

Figure 2.8 Variation of yaw moment with strake radial position, $\delta_{1}=\sigma^{\circ}, \alpha=50^{\circ}$. Reproduced from figure 7 of Malcolm (1991).

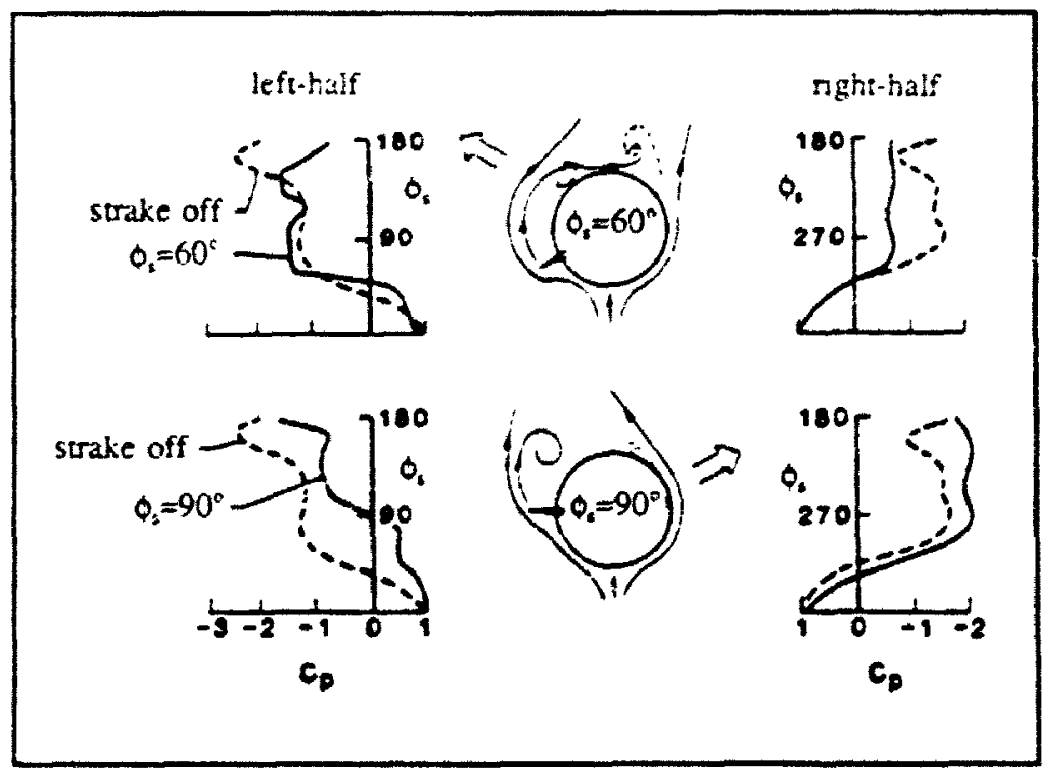

Figure 2.9 Pressure coefficients and cross flow schematics for a single strake at $\phi_{1}=60$ and $90^{\circ}, \delta_{0}=0^{\circ}, \alpha=50^{\circ}$. Reproduced from figure 8 of Malcolm (199I). 


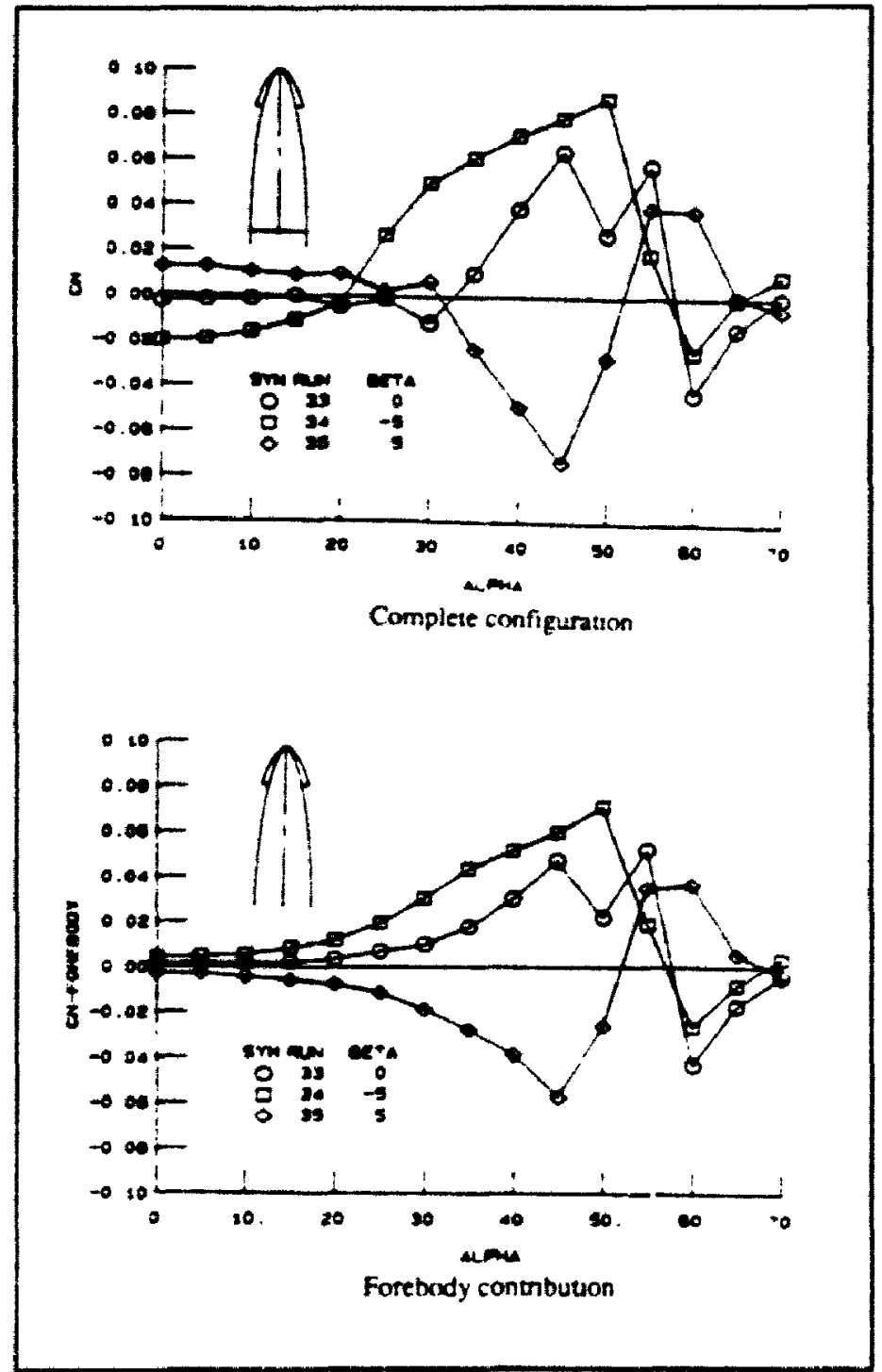

Figure 2.10(a) Yau moment coefficients for strakes positioned symmetrically at $\phi_{1}= \pm 150^{\circ}$. Reproduced from figure 18 of Malcolm (I991). 


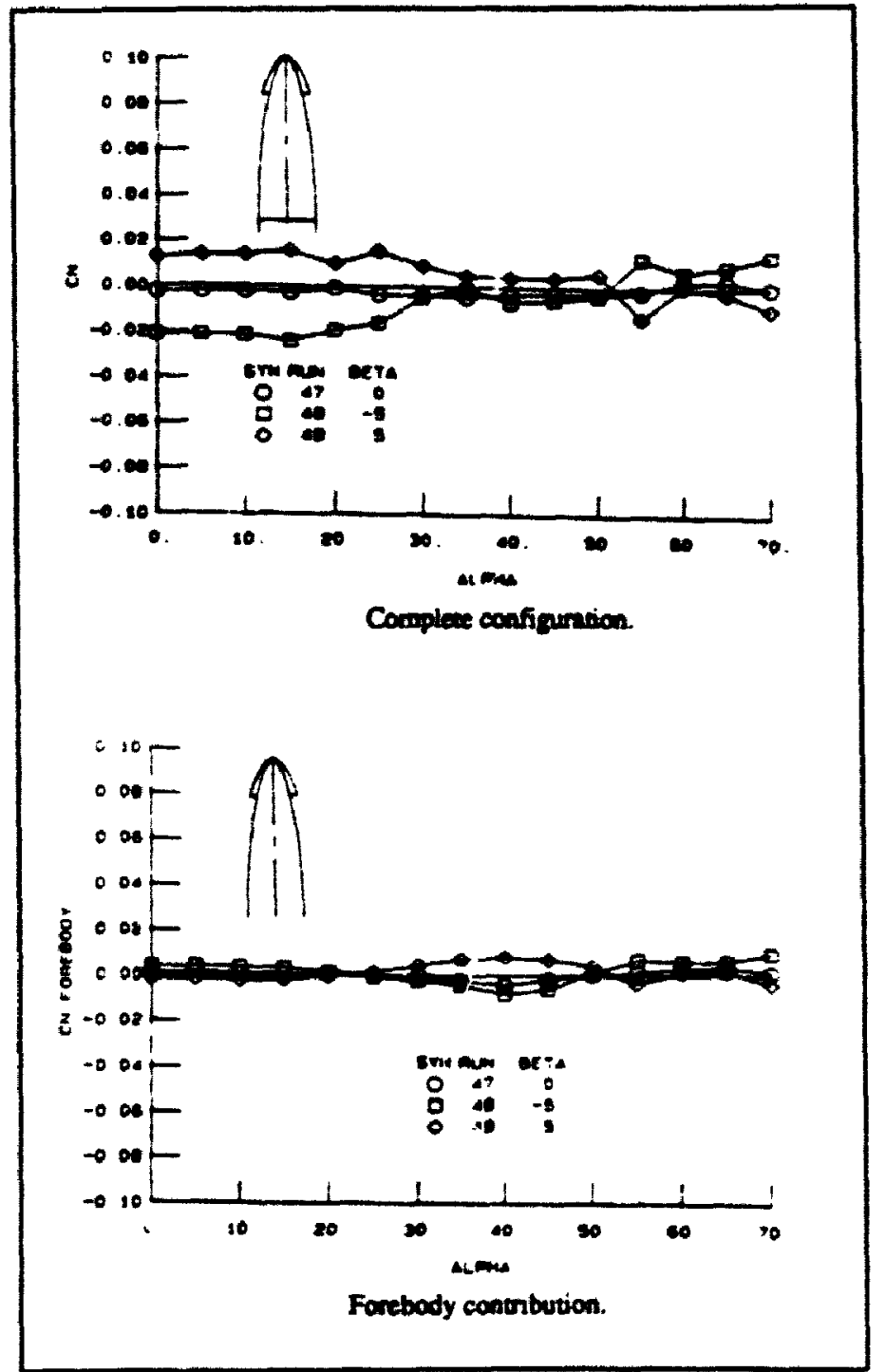

Figure 2.10(b) Yaw moment coefficients for strakes posttioned symmetrically at $\phi_{t}= \pm 105^{\circ}$. Reproduced from figure 19 of Malcolm (1991). 


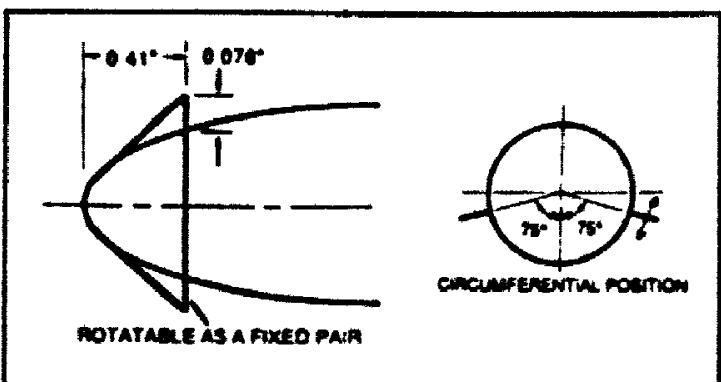

Figure 2.11 Schematic of rotatable nose tip strakes. Reproduced from figure 3 of Malcolm and $\mathrm{Ng}$ (199I).

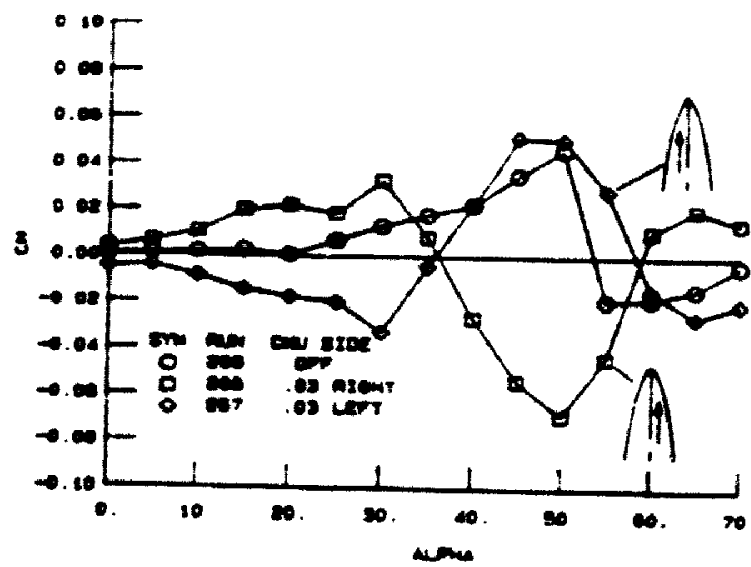

(a) $x / d=0.5$

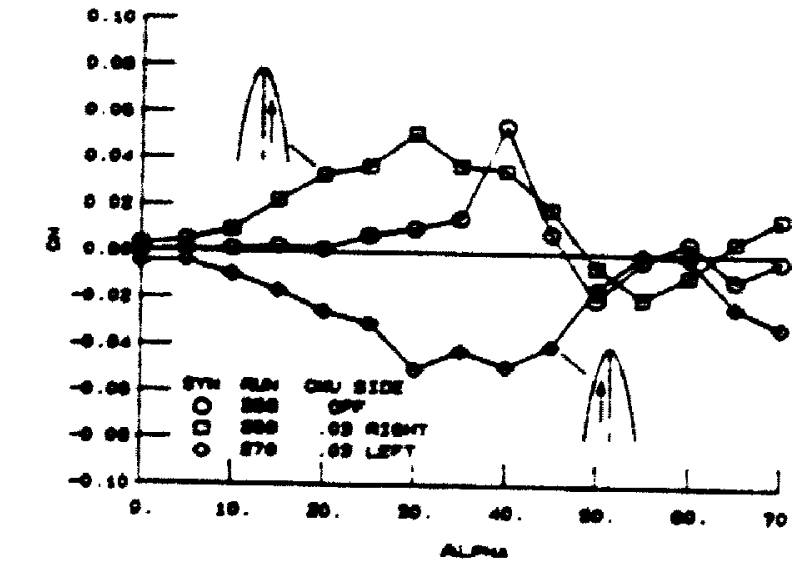

(b) $x / d=1.0$

Figure 2.12 Yaw moment versus angle of attack for forward blowing with nozzles at 2 different longitudinal stations, $x$ is the distance of the nozzles af of the apex. $d$ is the base diameter. Reproduced from figure 13 of Malcolm (1991). 


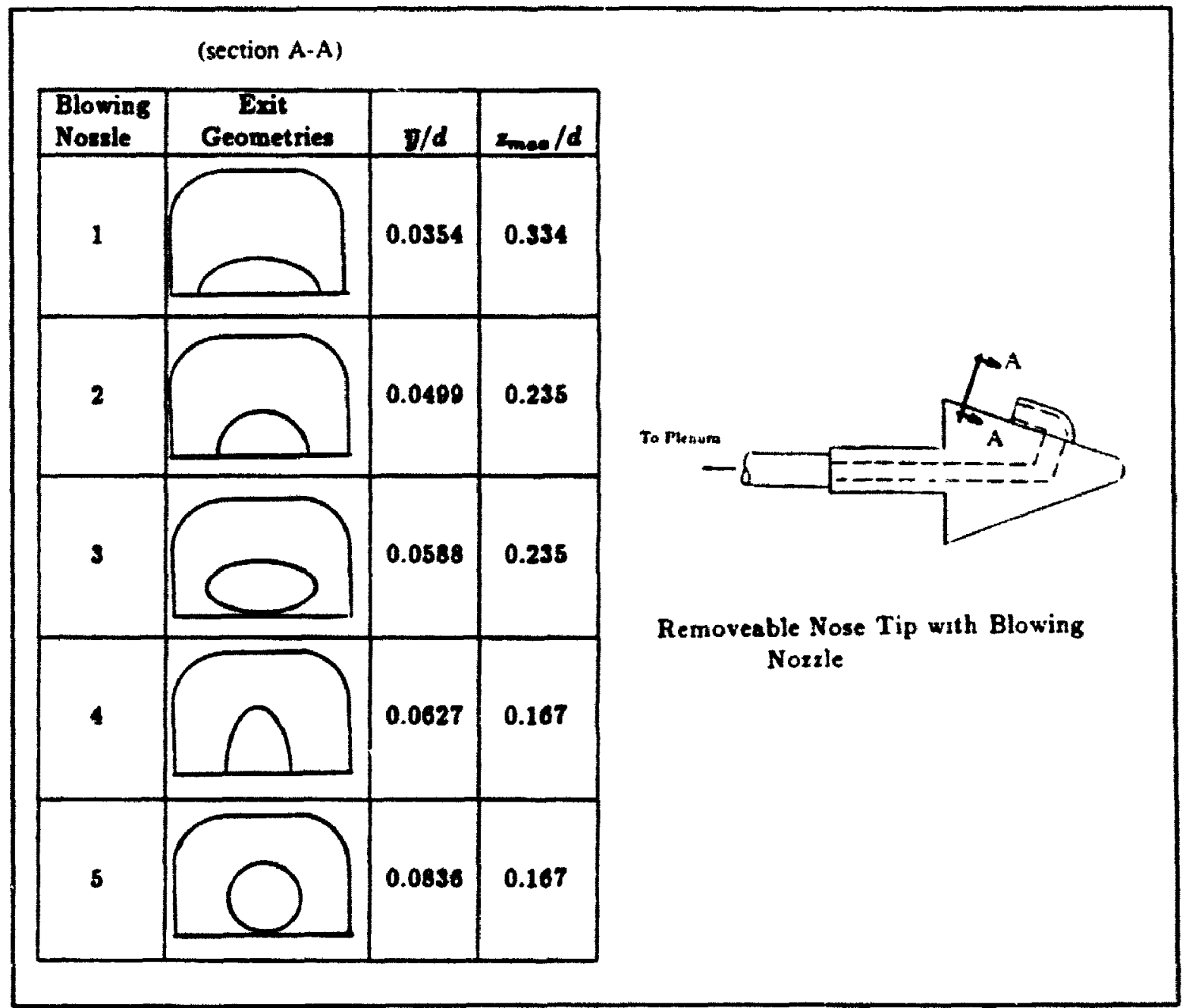

Figure 2.13 Various nozzle exit geometties tested by Gittner and Chokani (1992). y/d is the nozzle nondimensional geometric mean height, $z_{\text {max }} / d$ is the nondimensional width. Reproduced from figure 4 of Ginner and Chokani (1992). 


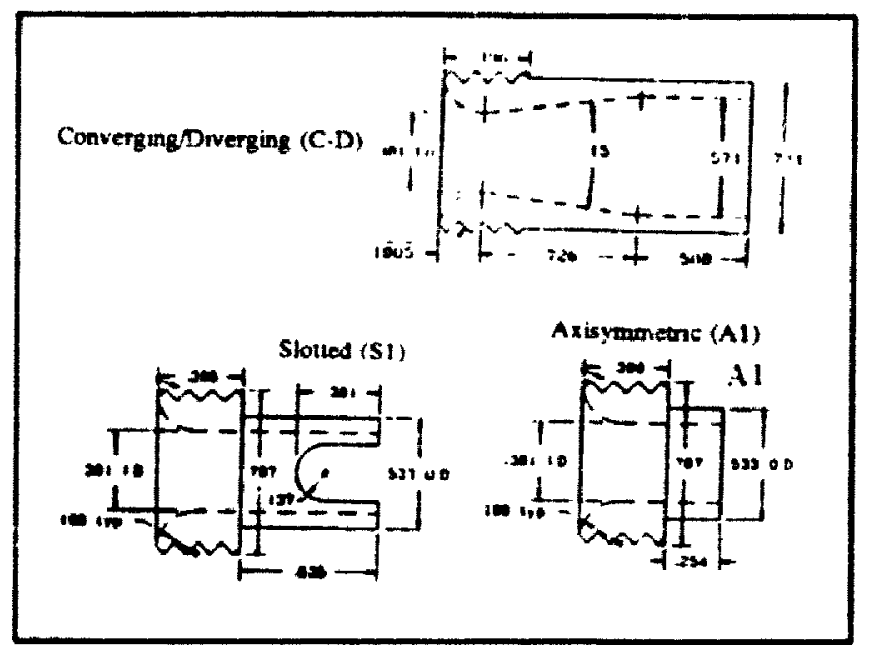

Fanure 2.14(a) Various nozzle exit geometries tested by Cornelius et al (1992). Reproduced from figure 13 of Corneliur et al (1992).

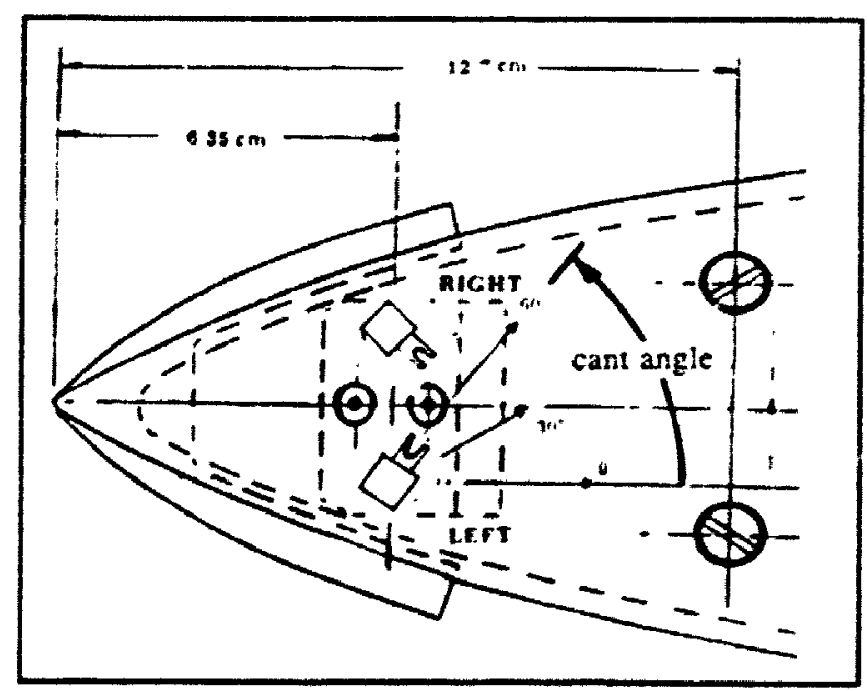

Fagure 2.14(b) Schematic defining exit nozzle orientation. Reproduced from figure 2 of Cornetius et al (1992). 


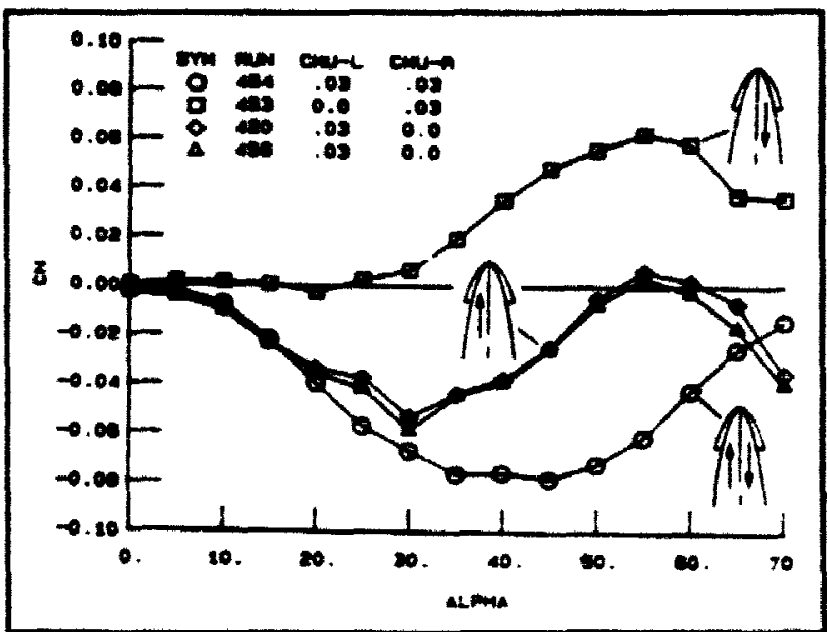

Figure 2.15 Yaw moment versus angle of attack for combined forward and aft blowivg with symmetric strakes. Reproduced from figure $/ 1$ of Malcolm and $\mathrm{Ng}$ (1991).

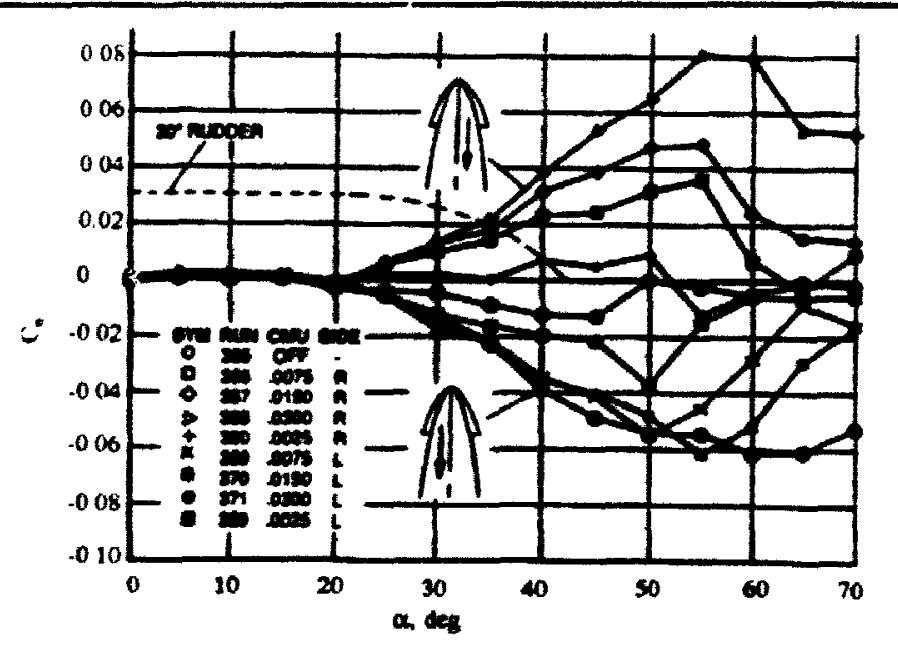

Figure 2.16 Yaw moment versus angle of artack for symmetric strakes with af tangential blowing. Reproduced from figure 9 of Malcolm and $N_{g}$ (1991). 


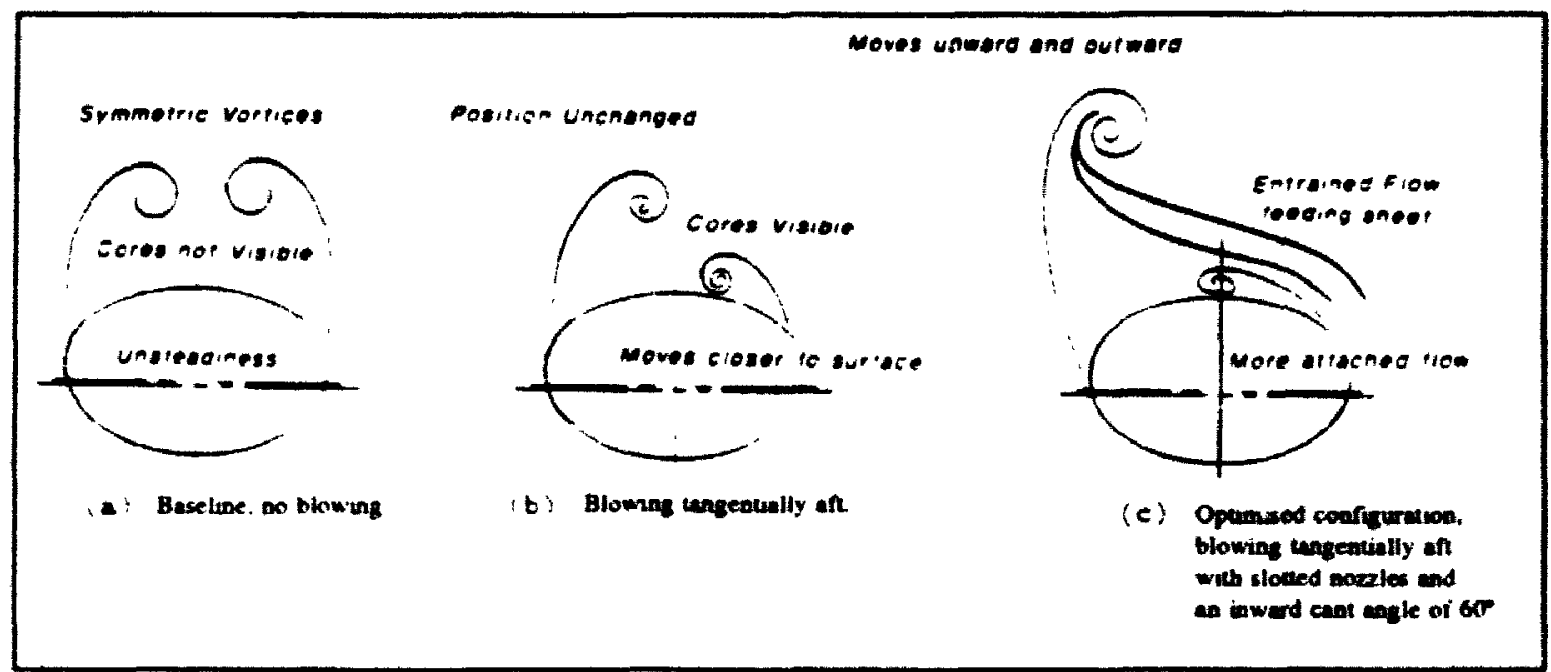

Figure 2.17 Comparison of flowfield schematics for various nozzle jet blowing configurations. Repraduced from figure 18 of Guvton and Maerki (1992).

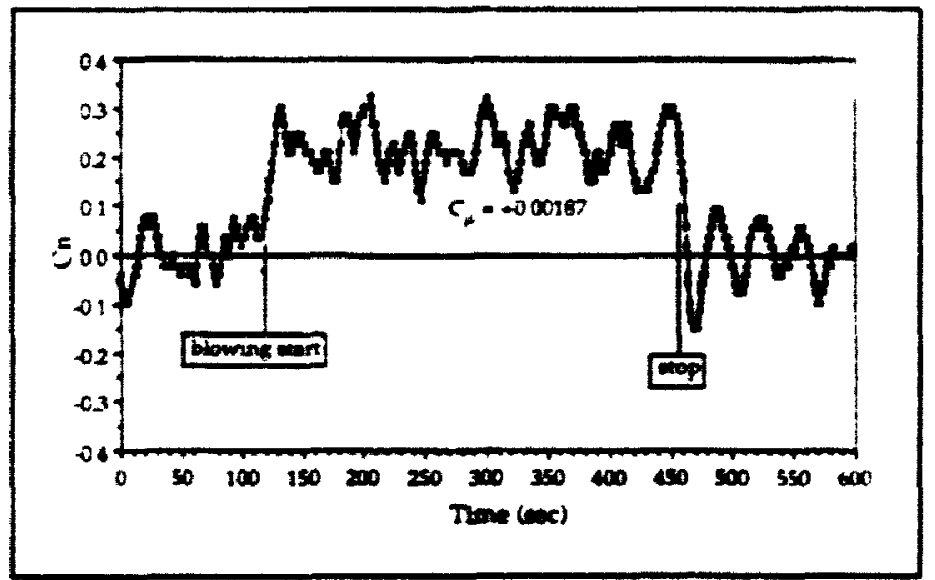

Figure 2.18(a) Transient behaviour of the yaw moment coefficient for an aft blowing jet, $\alpha=50^{\circ}$. Repraduced from figure 6 of Malcolm and $\mathrm{Ng}$ (1991). 


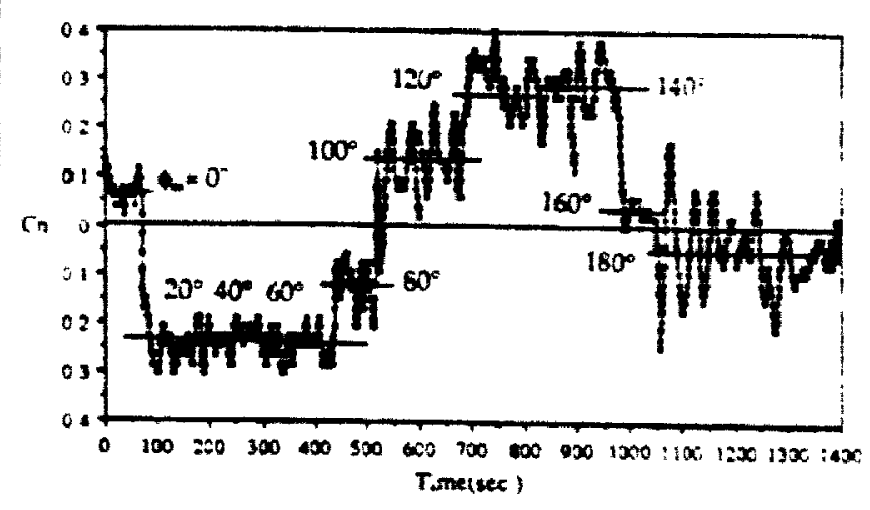

Figure 2.18(b) Transient behaviour of the yax moment coefficient for a miniature rotatable nose tip strake rotation, $\alpha=50^{\circ}$. Reproduced from figure 7 of $\mathrm{Ng}_{\mathrm{g}}$ and Malcolm (199/b).

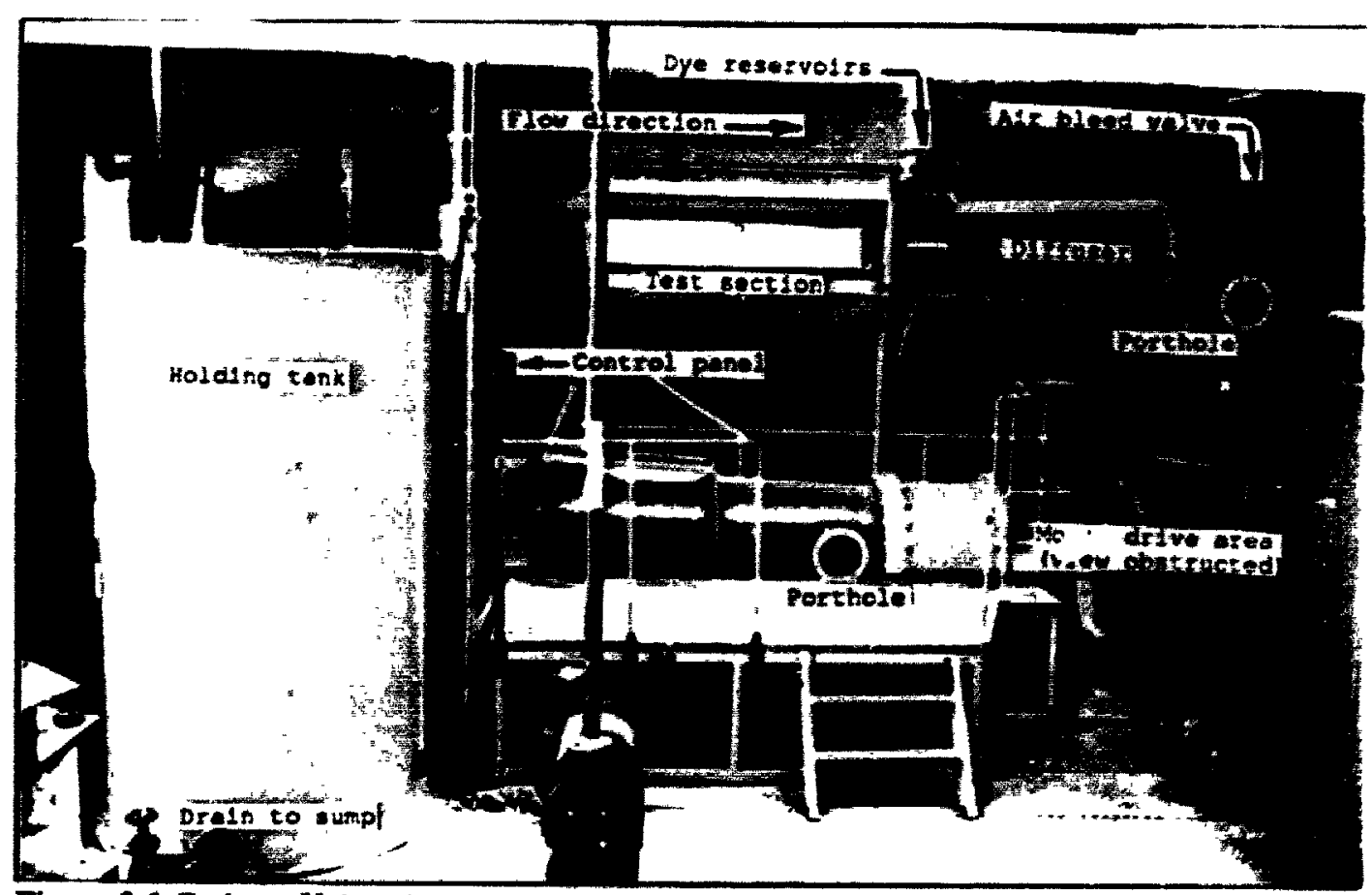

Figure 3.1 Carleton University water tunnel test facility. 


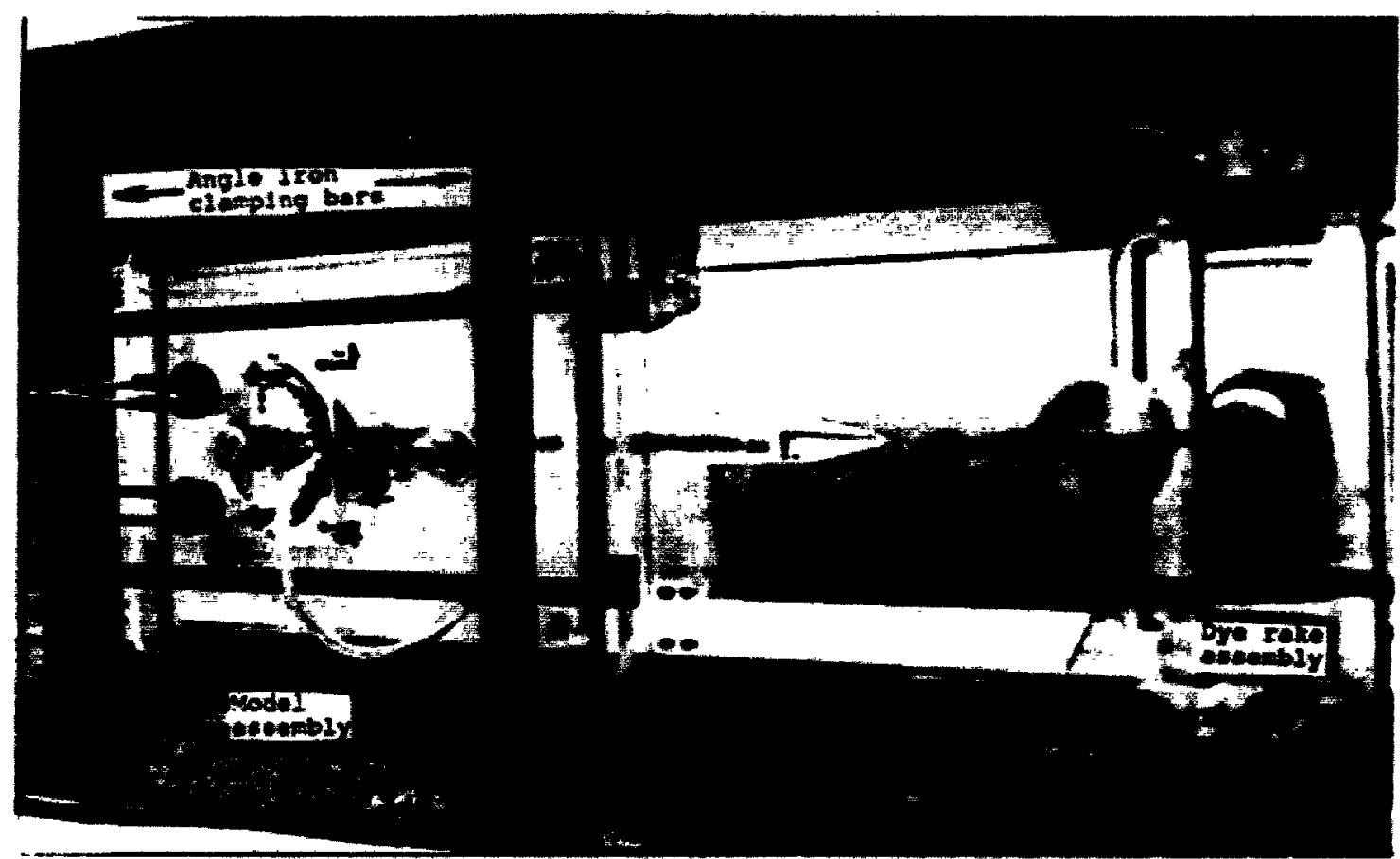

Fingure 3.2 Top view of test rection showing model-asppot insert.

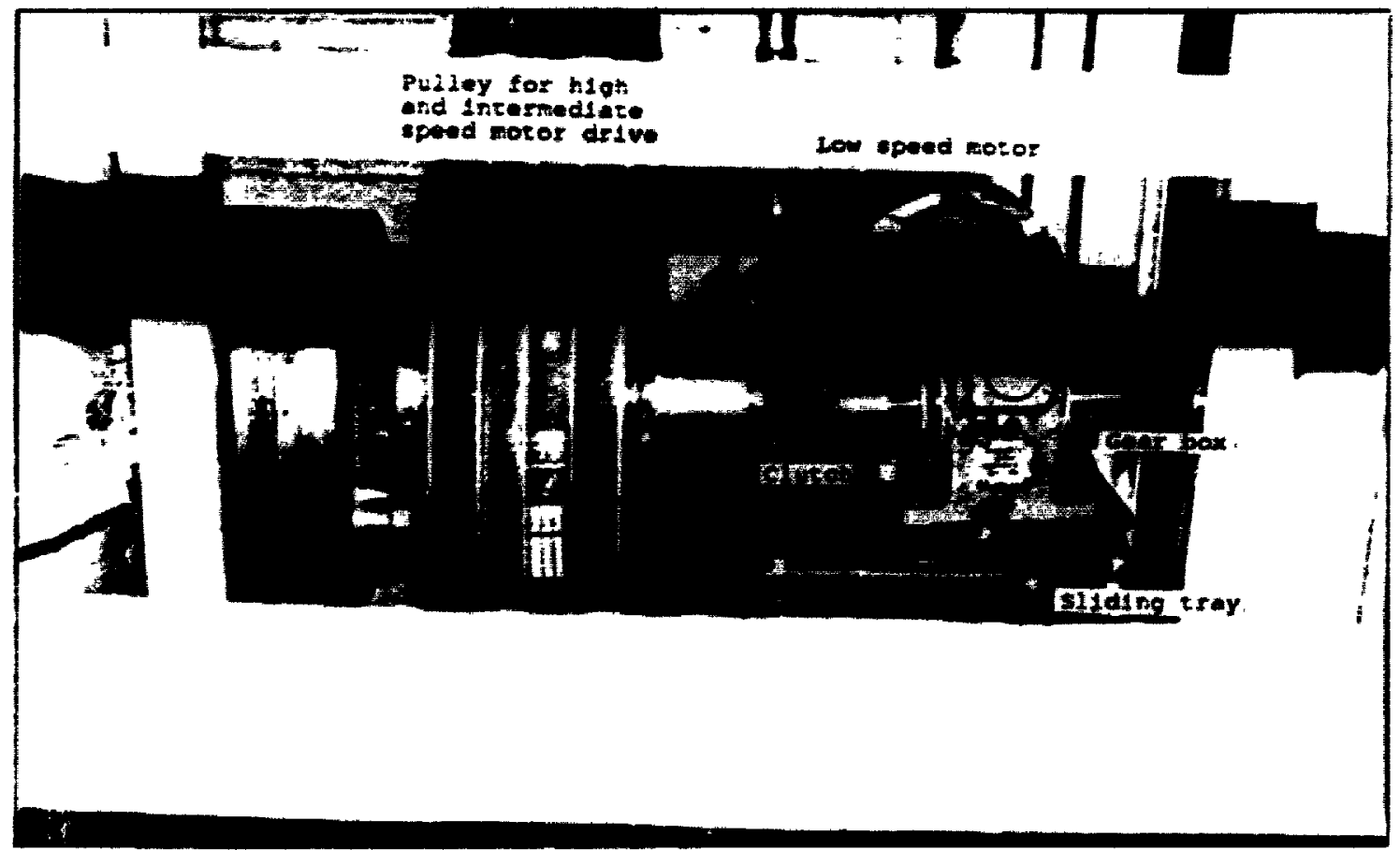

Figure 3.3 Low speed drive motor, gearbox and clutch. 


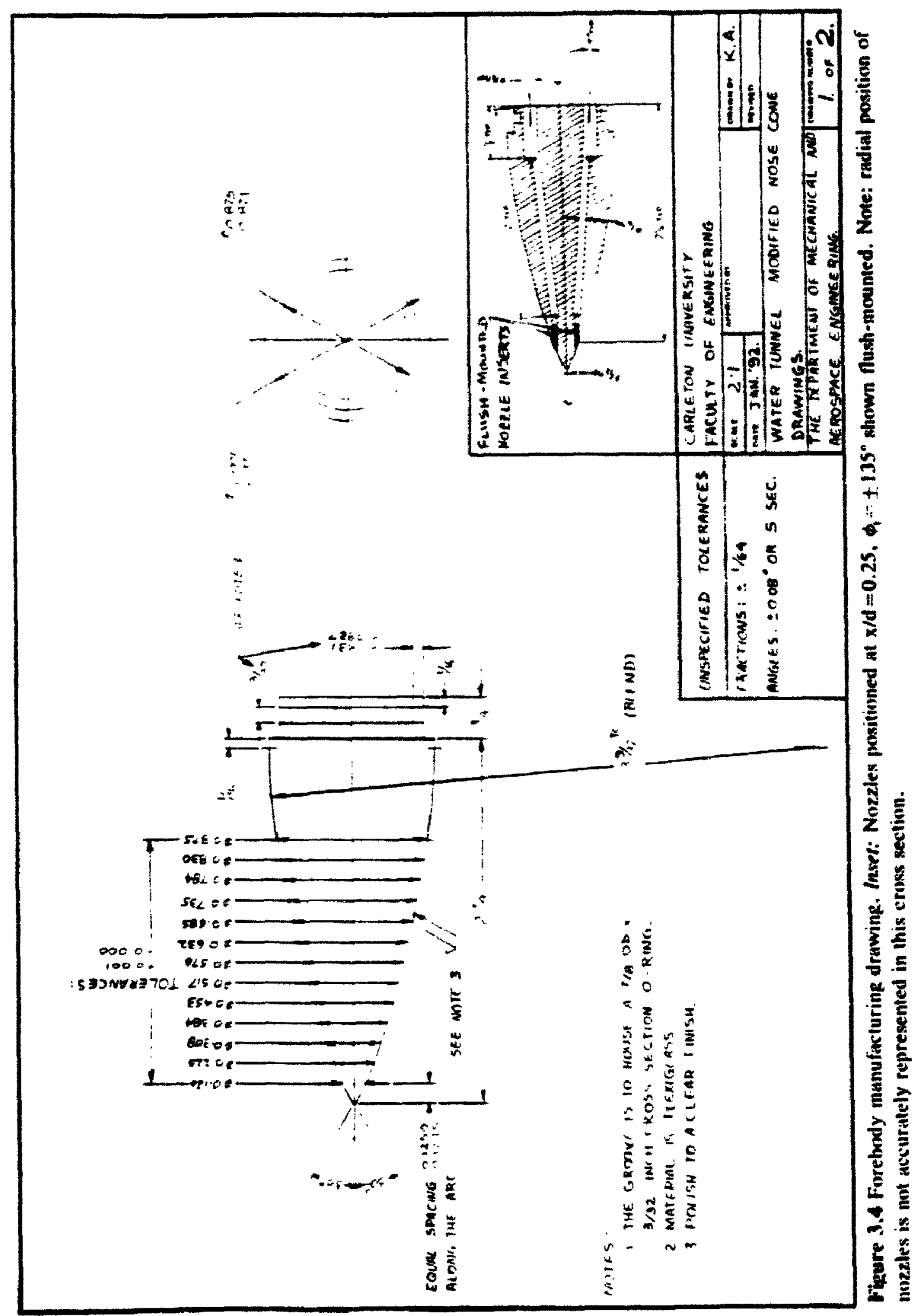




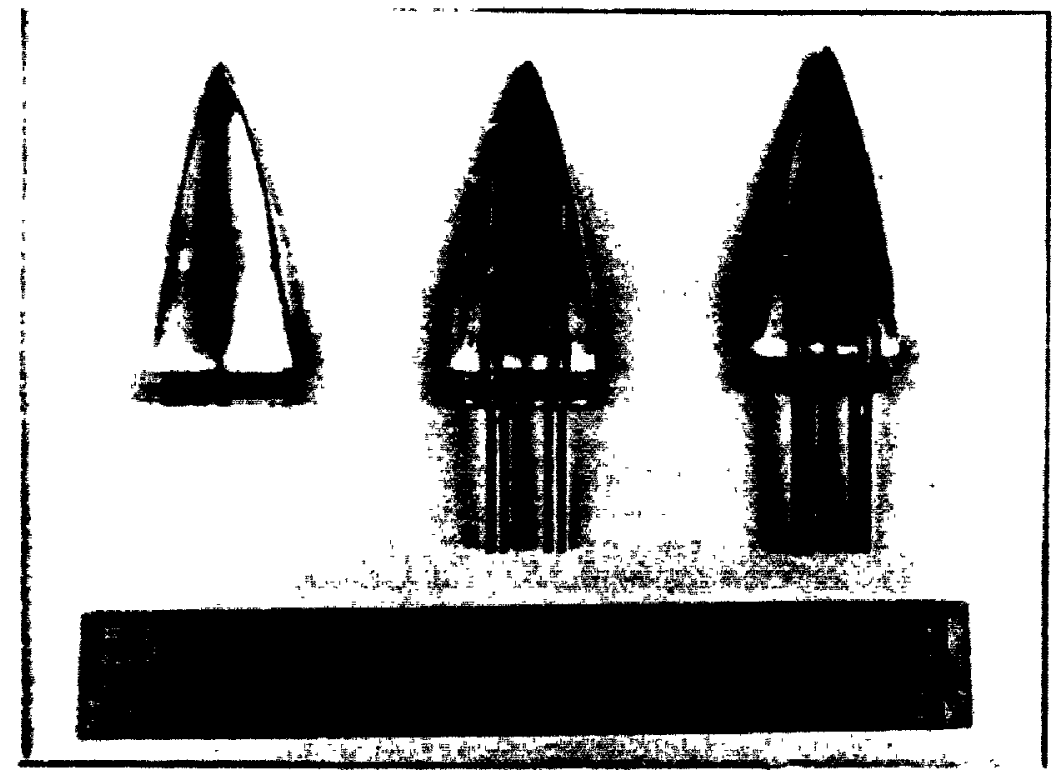

Figure 3.5 Various notecones used in experiments. Unmodified (left), nozzles at $x / d=1.0$ (centre), nozzles at $x / d=0.25$ (right).

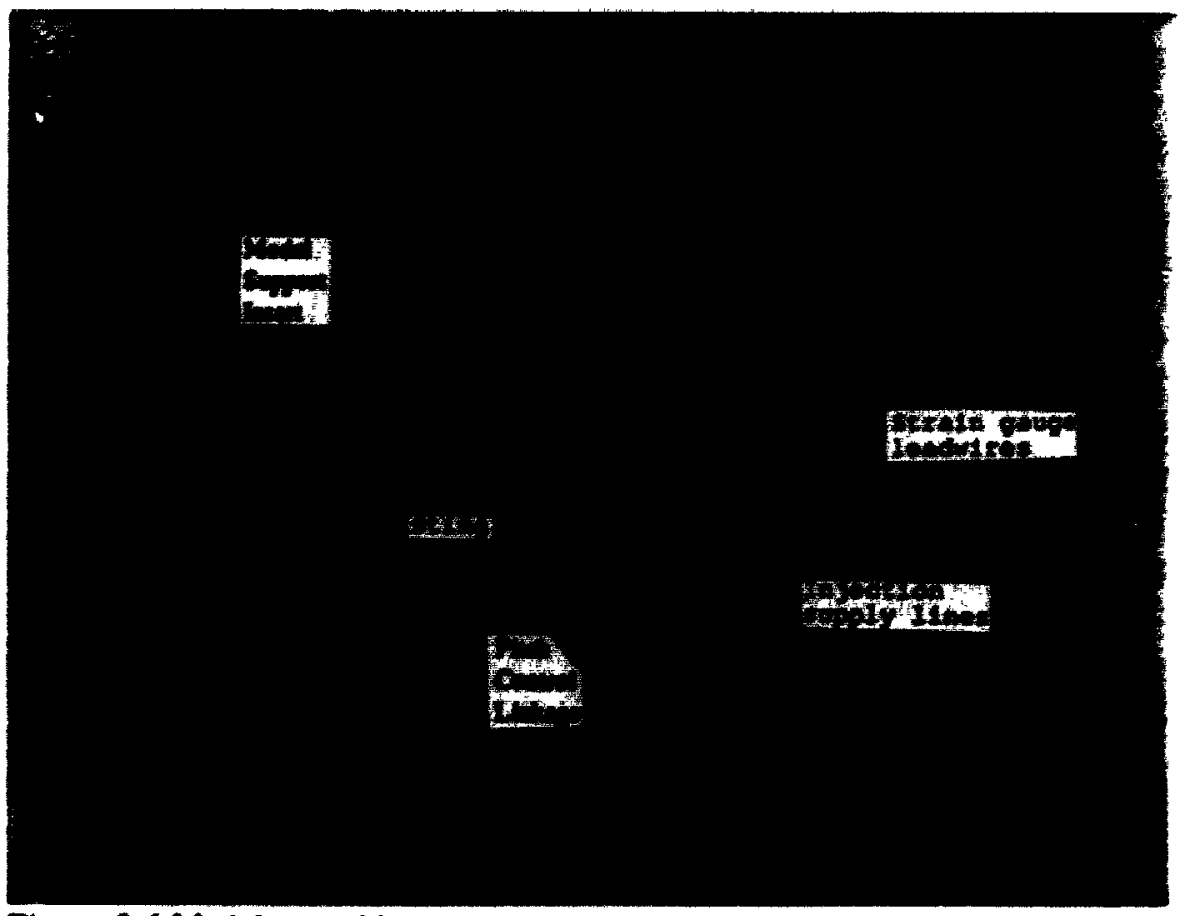

Fizure 3.6 Model assembly. 


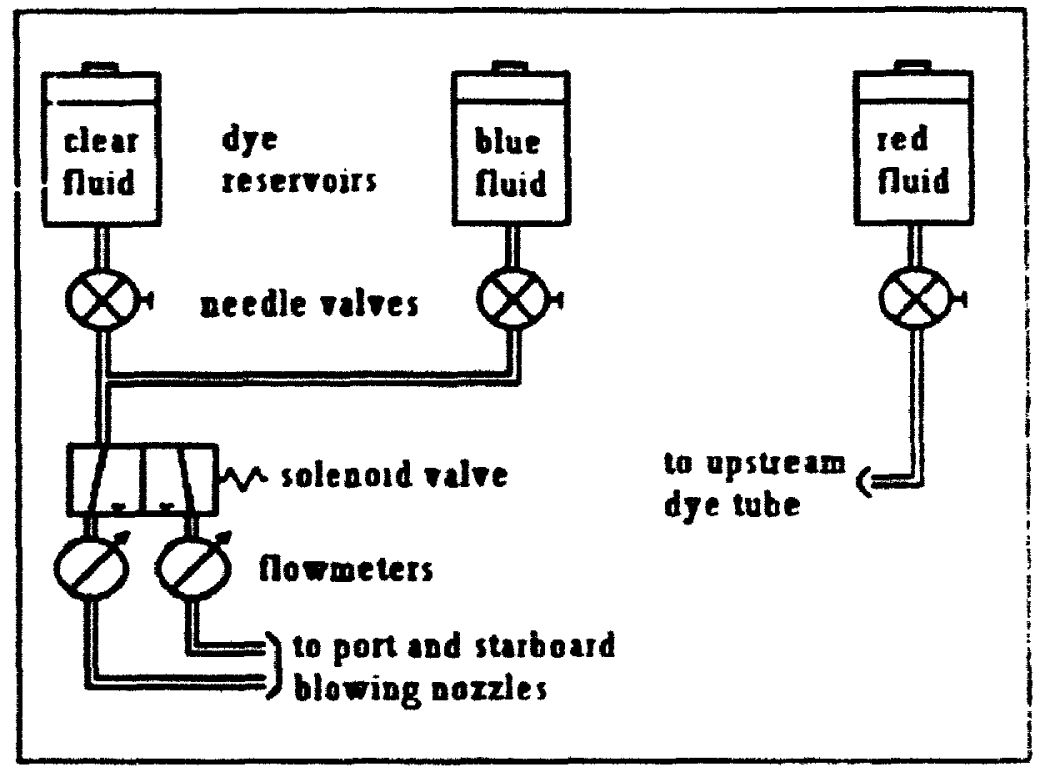

Figure 3.7 Schematic of injection plumbing configuration

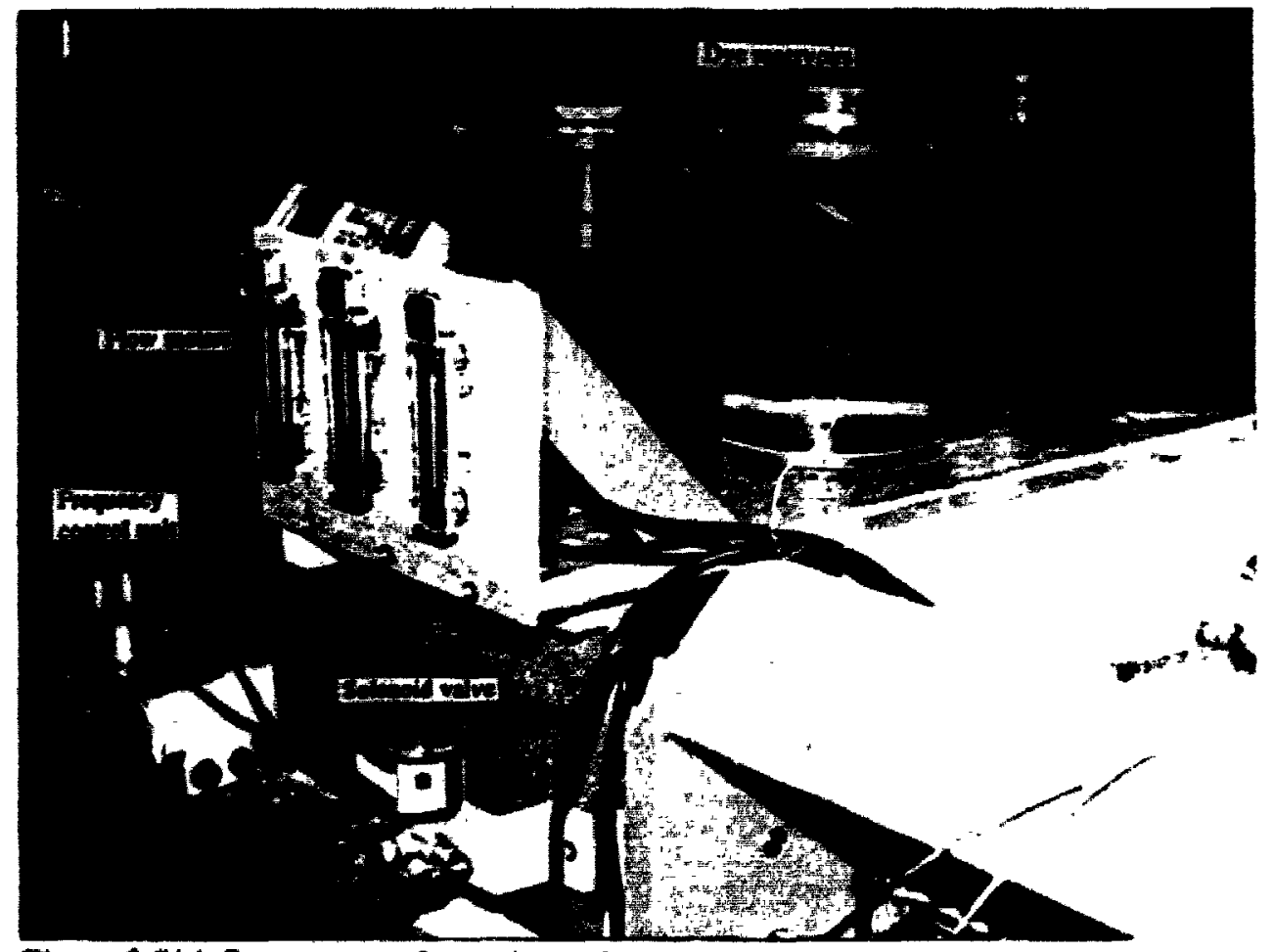

Figure 3.2(a) Components of experimental setup. 


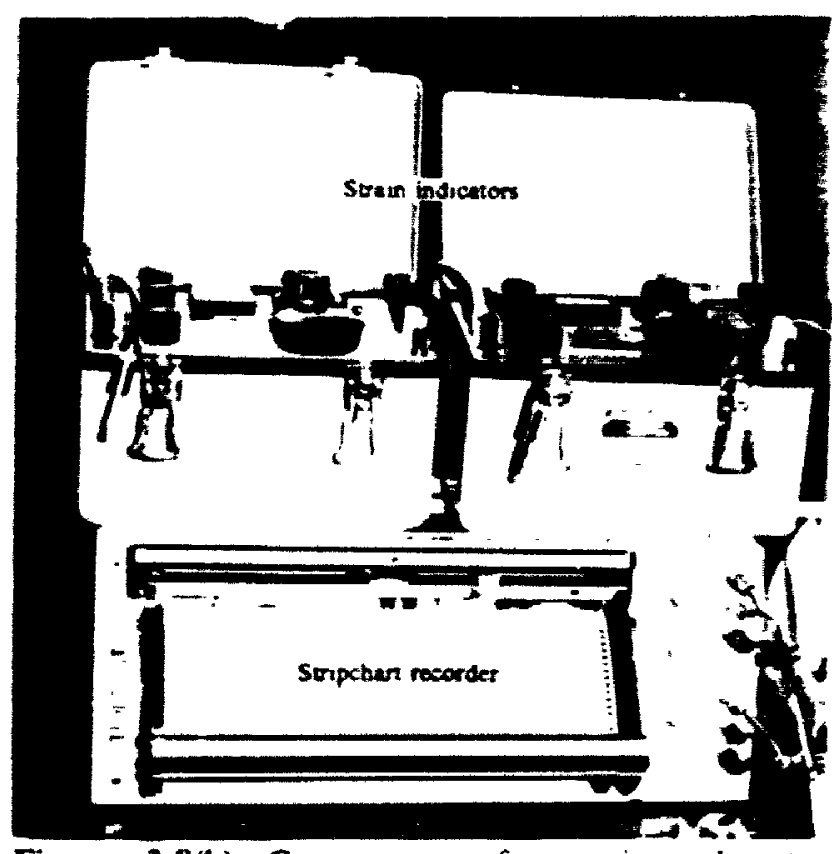

Figure 3.8(b) Components of experimental setup contmued

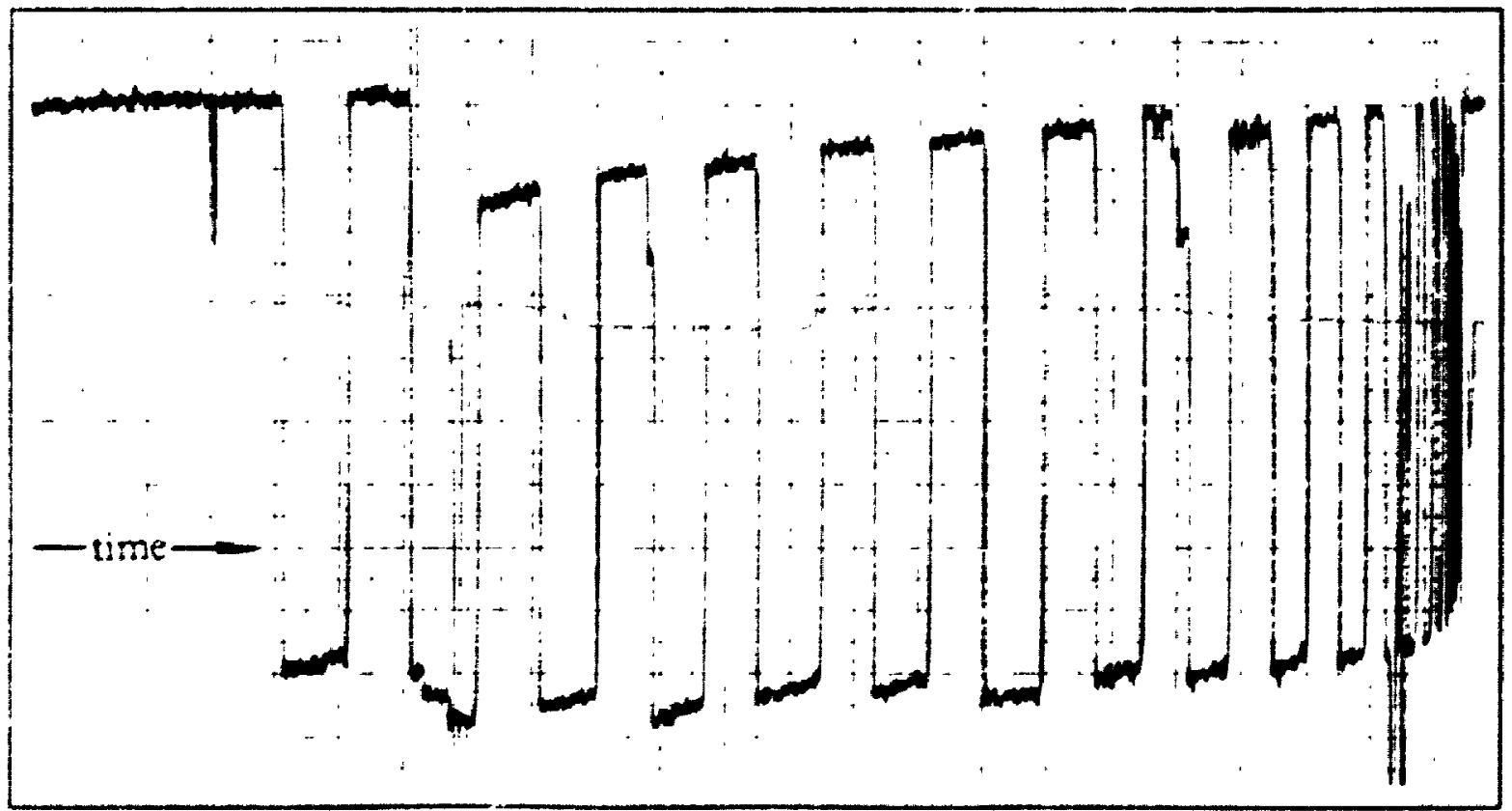

Figure 3.4 Sc ware wave signal indialiy piched up by the strapchart re order before appropriate shielding was

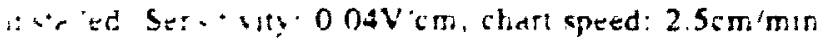




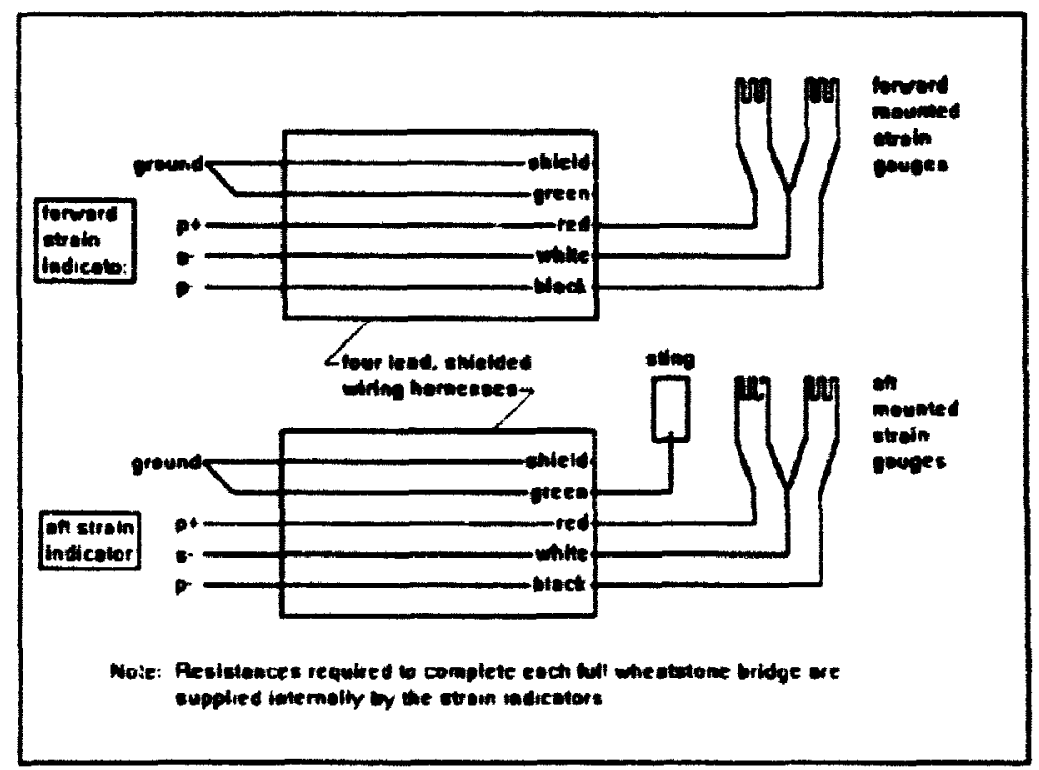

Figure 3.10 Schematic of strain gauge wirng configuration.

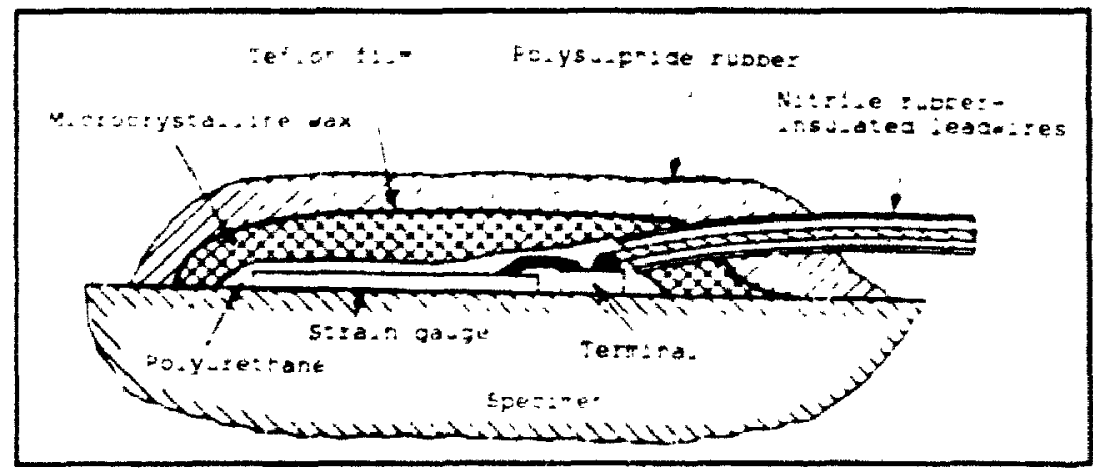

Fiqure 3.11(a) Waterproofing of strain gauges. Reproduced from figure 6.23 of Dall! and Riley (1991). 


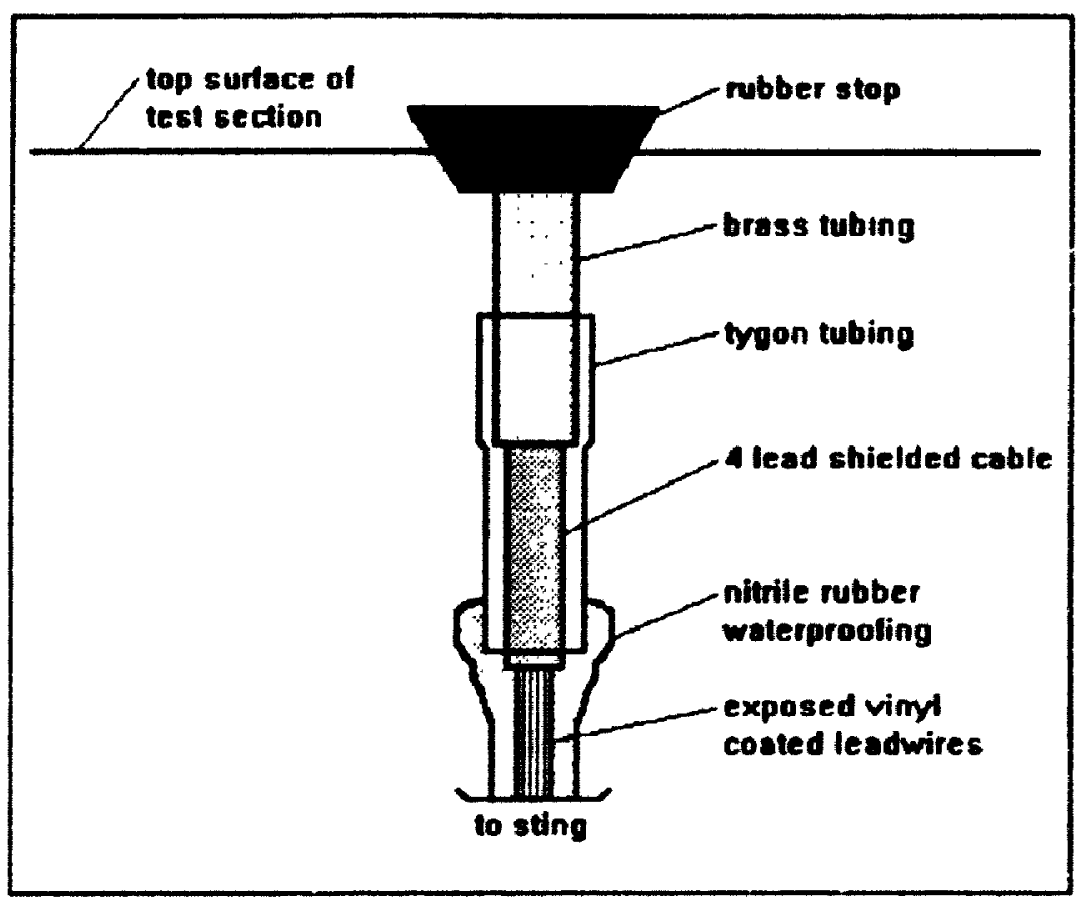

Figure 3.11(b) Wateiproofing of strain gauge leaduires.

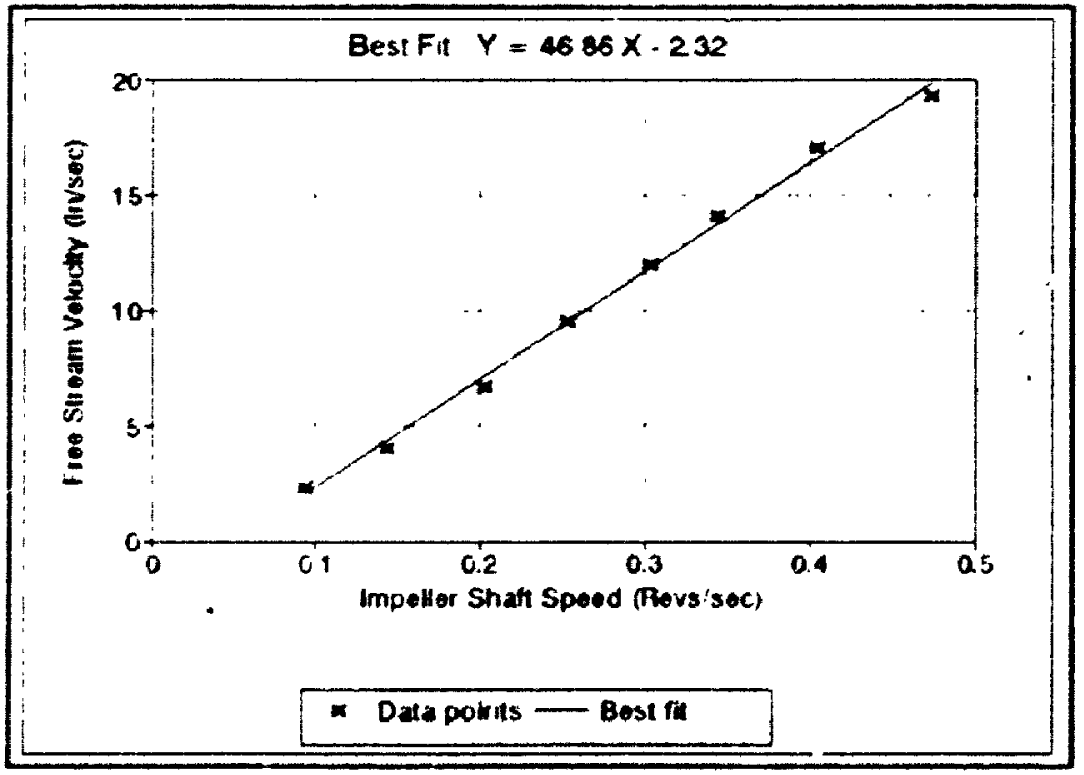

Figure 3.12 Free stream velocity calibration curve. 


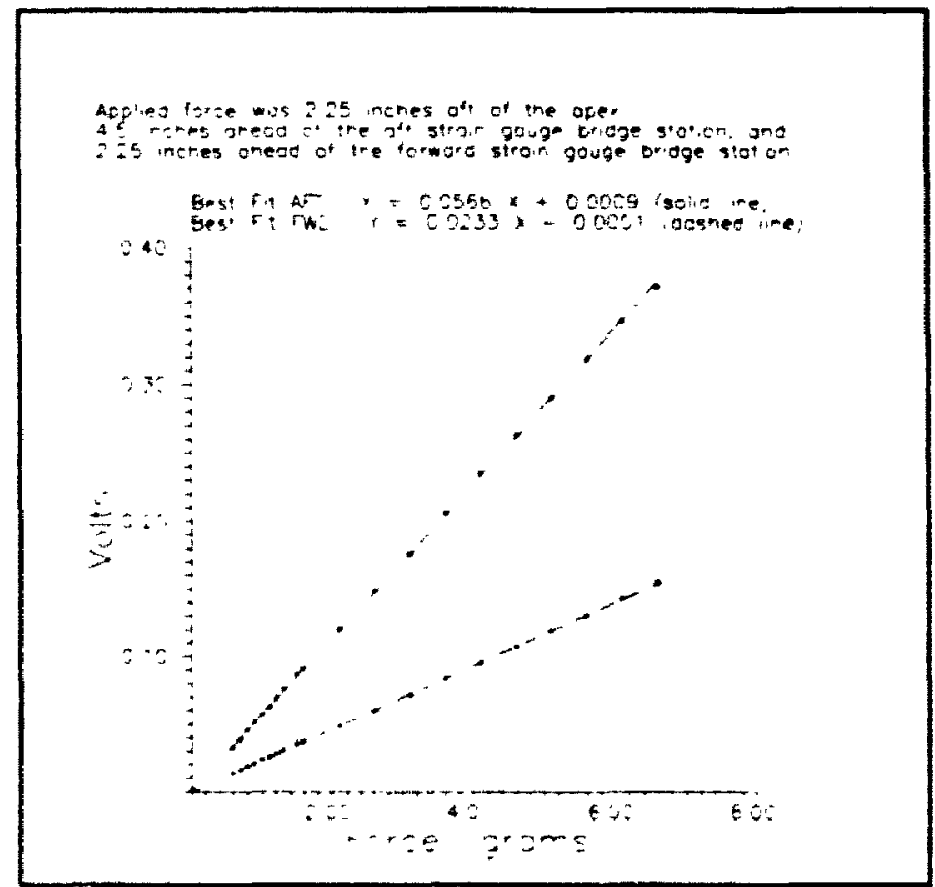

Figure 3.13 Voltage output versus applied calibration force for forward and aft mounted strain gauge bridge stations.

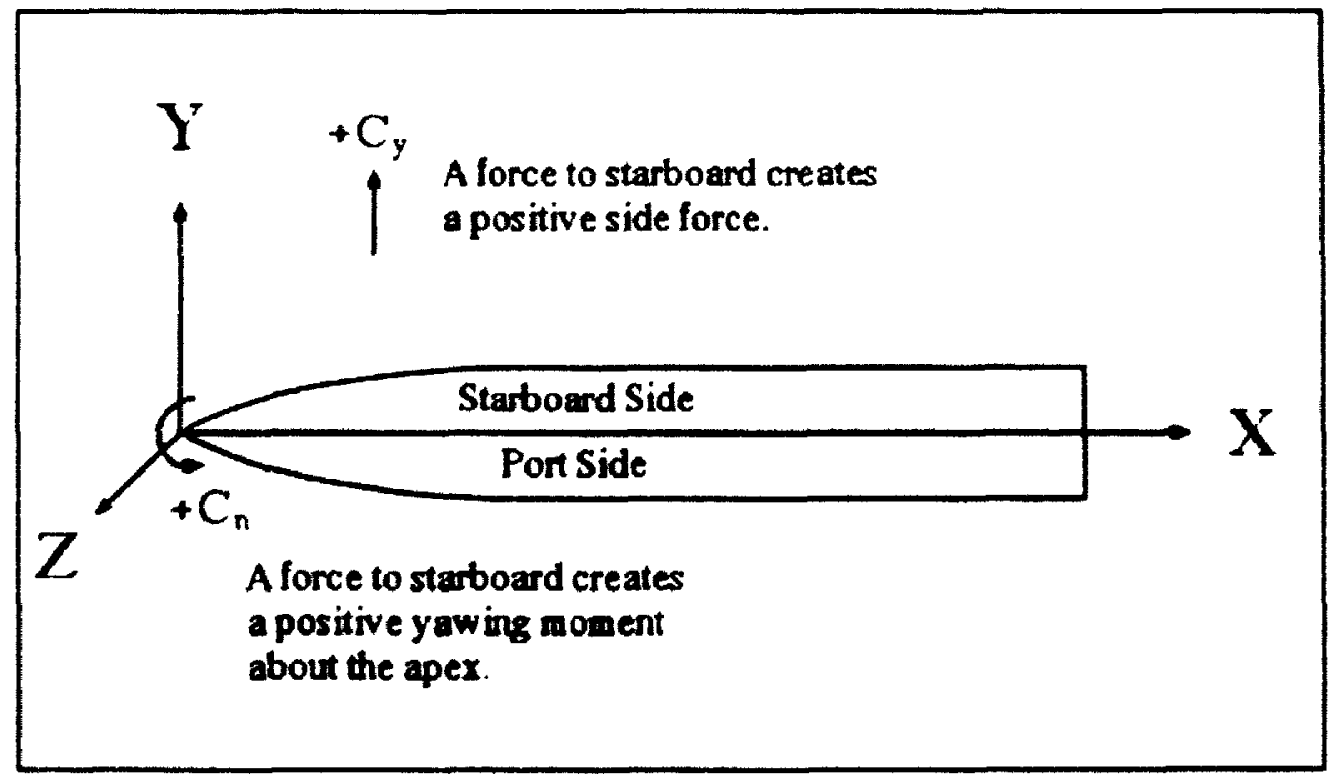

Figure 4.1 Schematic of adopted sign convention, plan view. 


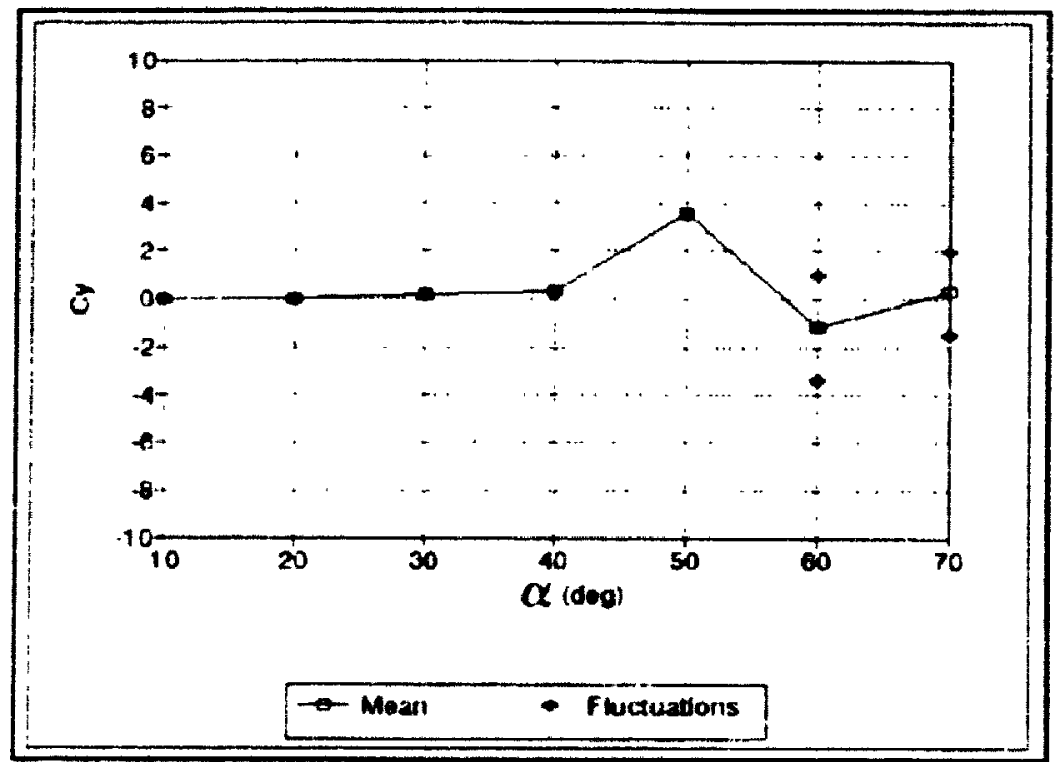

Figure $4.2(a)$ Side force versus angle of attack for the clean-forebody. $\beta=0, \operatorname{Re}_{d}=6700$.

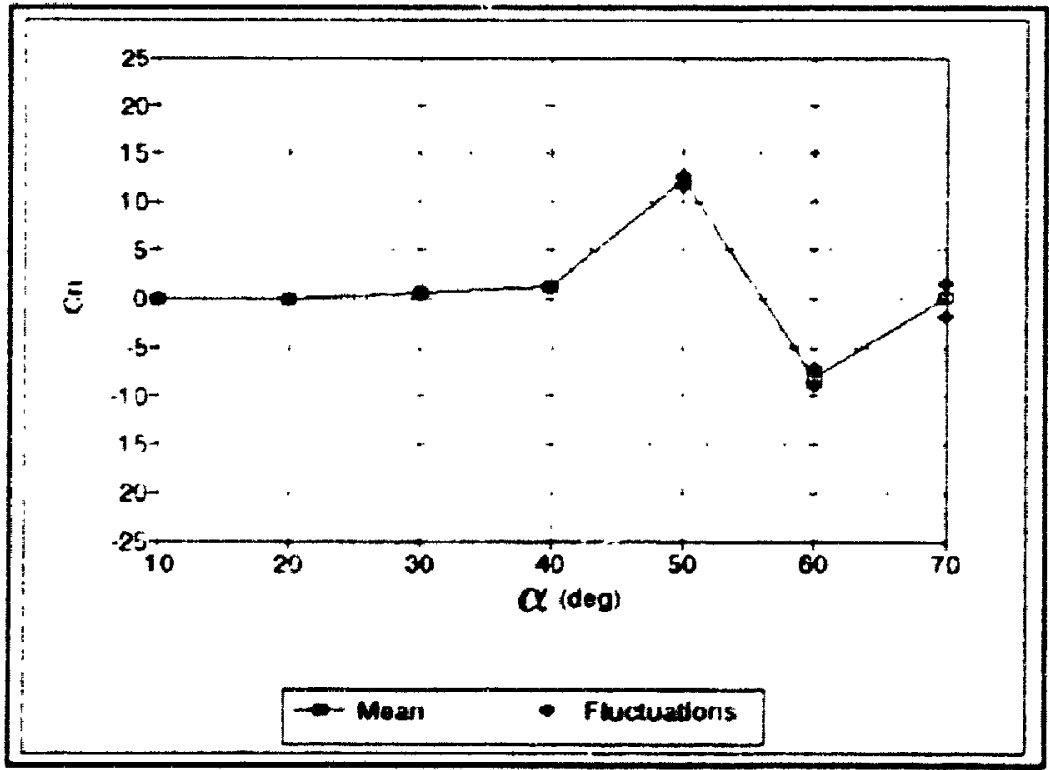

Figure 4.2(b) Yaw moment versus angle of attack for the clean-forebody. $\beta=0^{n}, \operatorname{Re}_{d}=6700$. 


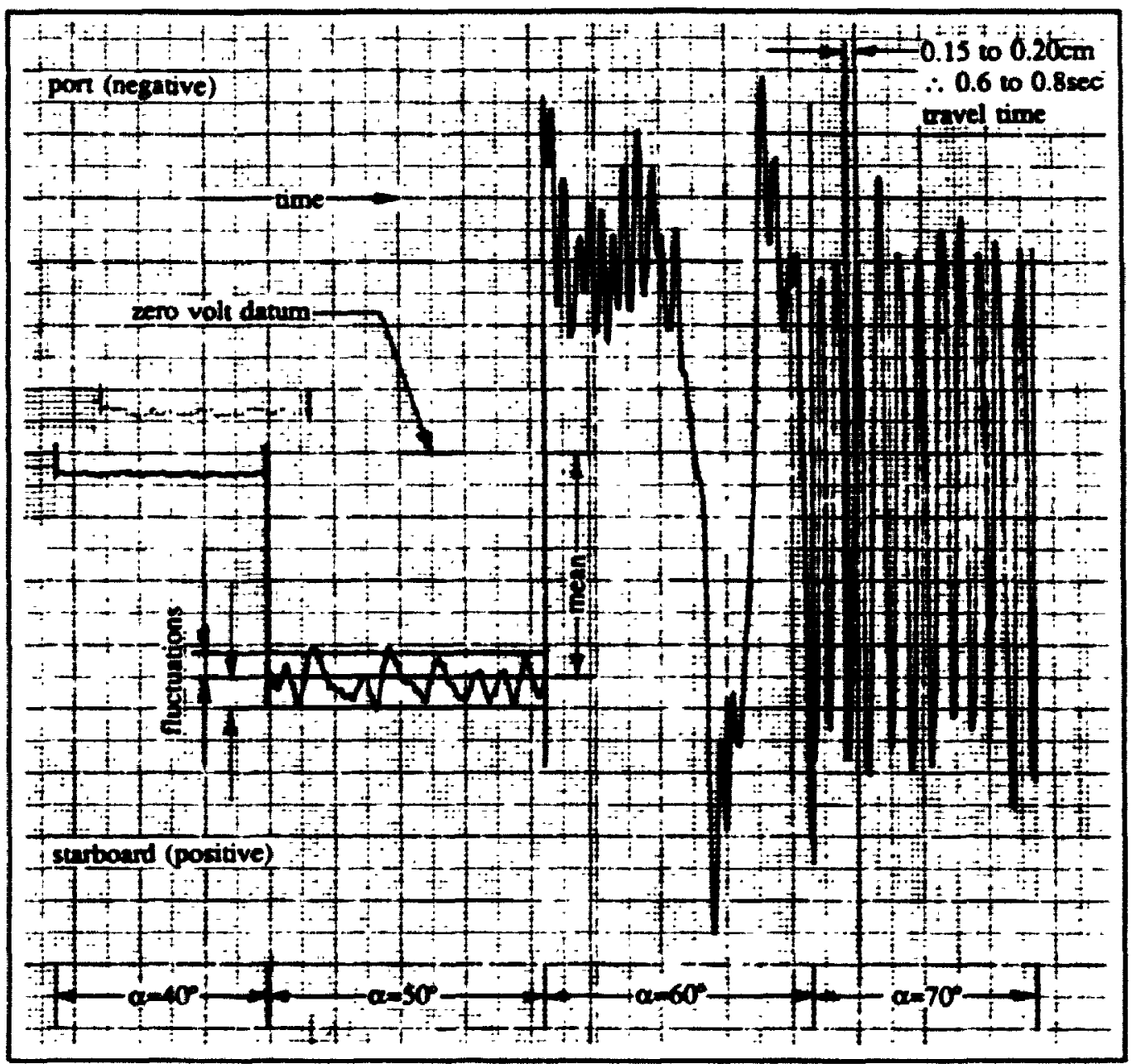

Fagure 4.2(c) Forward strain gauge bridge station stripchart output for the clean-forebody flowfield. $\beta=\sigma, R_{d}=6700$, sensitivity: $0.04 \mathrm{~V} / \mathrm{cm}$, chart speed: $15 \mathrm{~cm} / \mathrm{min}$. 


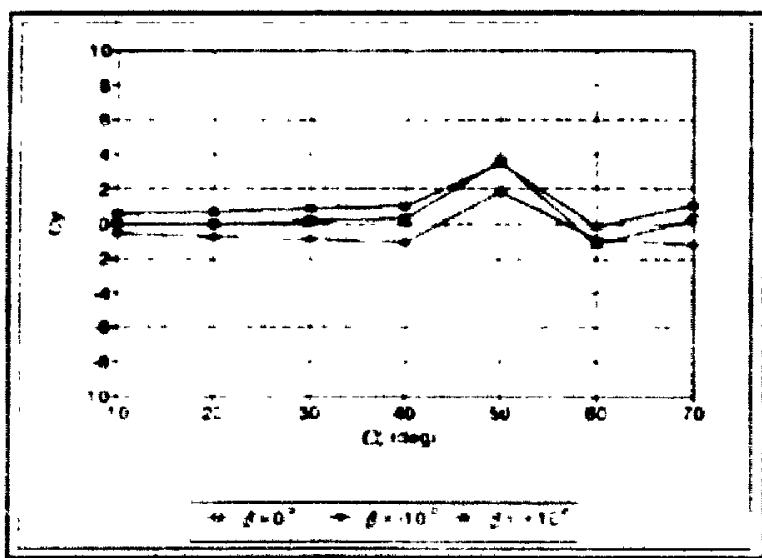

Fipure \$.3(a) Mean side torce versus angle of attack for the clean-forebody. $R e_{d}=6700$.

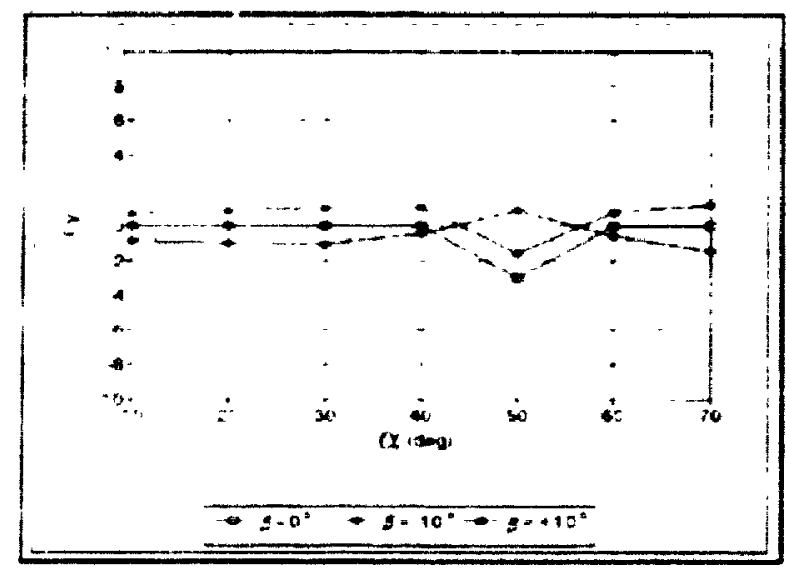

Figure 4.4(a) Mrat: sde tors iersus angle ot aiksth

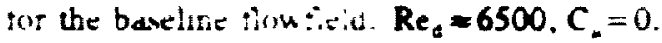

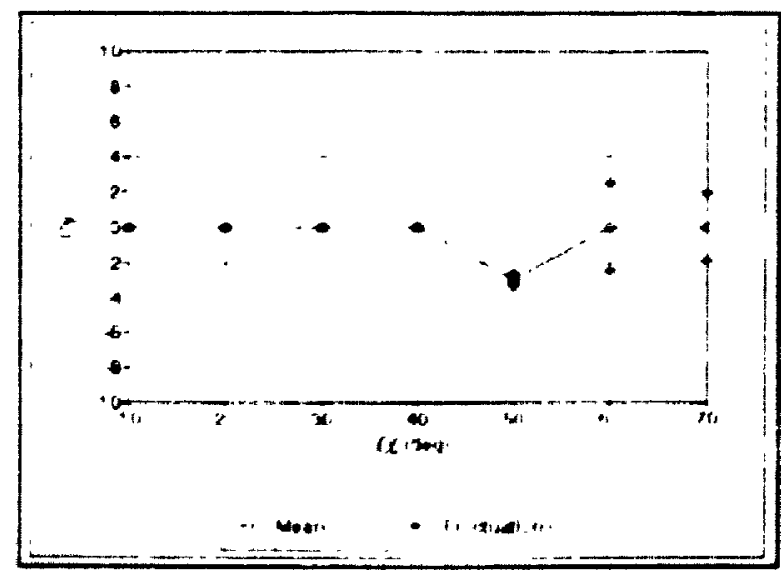

Figure $\mathbf{4 . 5 ( a )}$ Side torce versus angle of attack for the baseline flowfield. $B=0^{\circ}$. $\mathrm{Re}_{3} \approx 6500 . \mathrm{C}_{*}=0$.

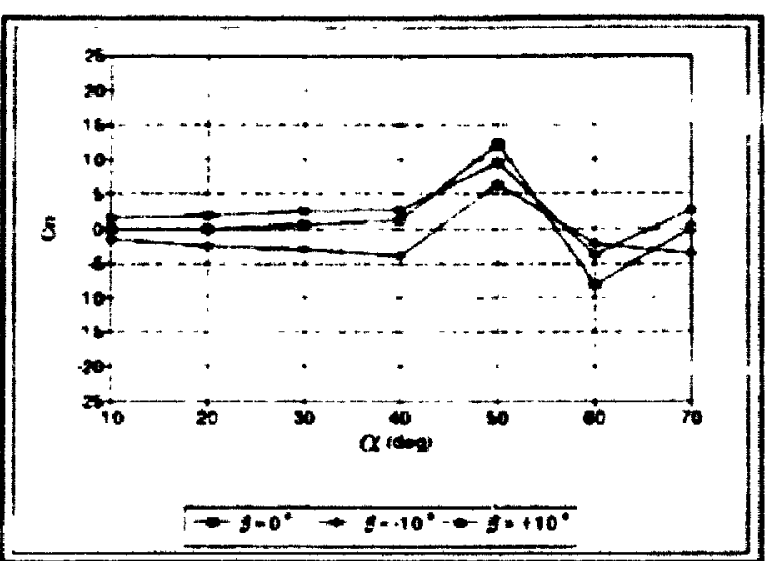

Figure 4.3(b) Mean yaw moment versus angle of attack for the clean-forebody. $R_{e}=6700$.

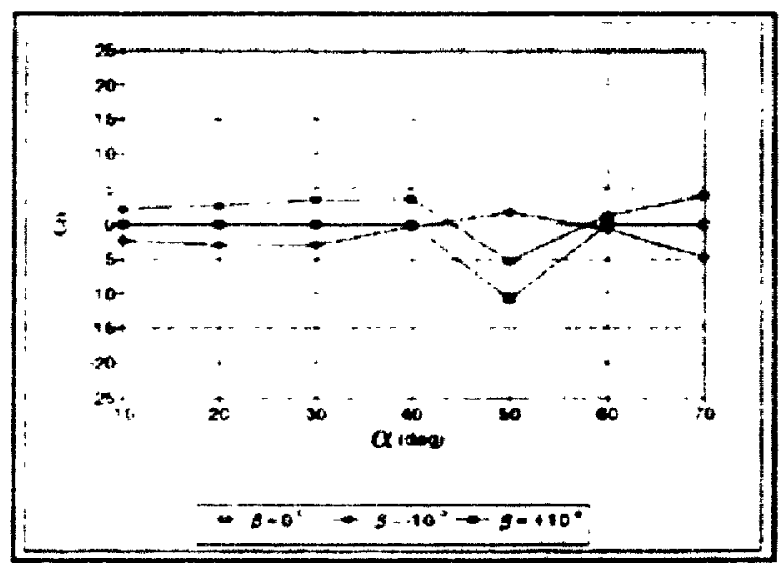

Fiqure 4.t(h) Mea: yah moment versus angle of attack for the baseline thoufield. $R_{d}=6500, C_{s}=0$.

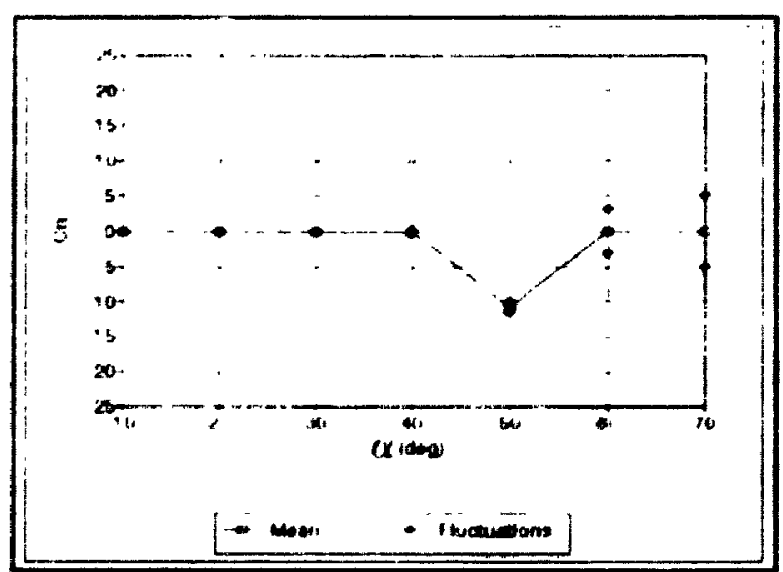

Figure 4.S(b) Yaw moment versus angle of attack for the baseline nowfield. $B=0, R_{t_{j}} \approx 6500, C_{\mu}=0$. 


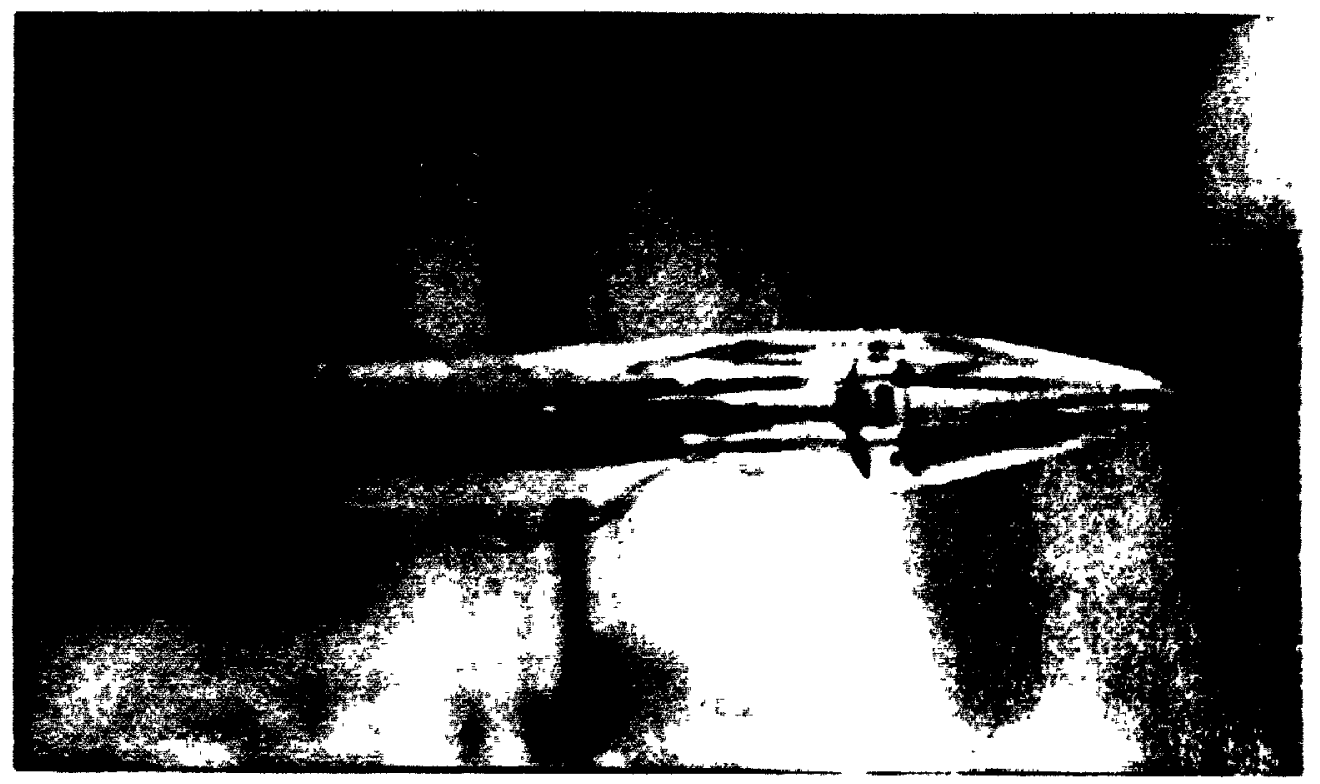

(a) Top view.

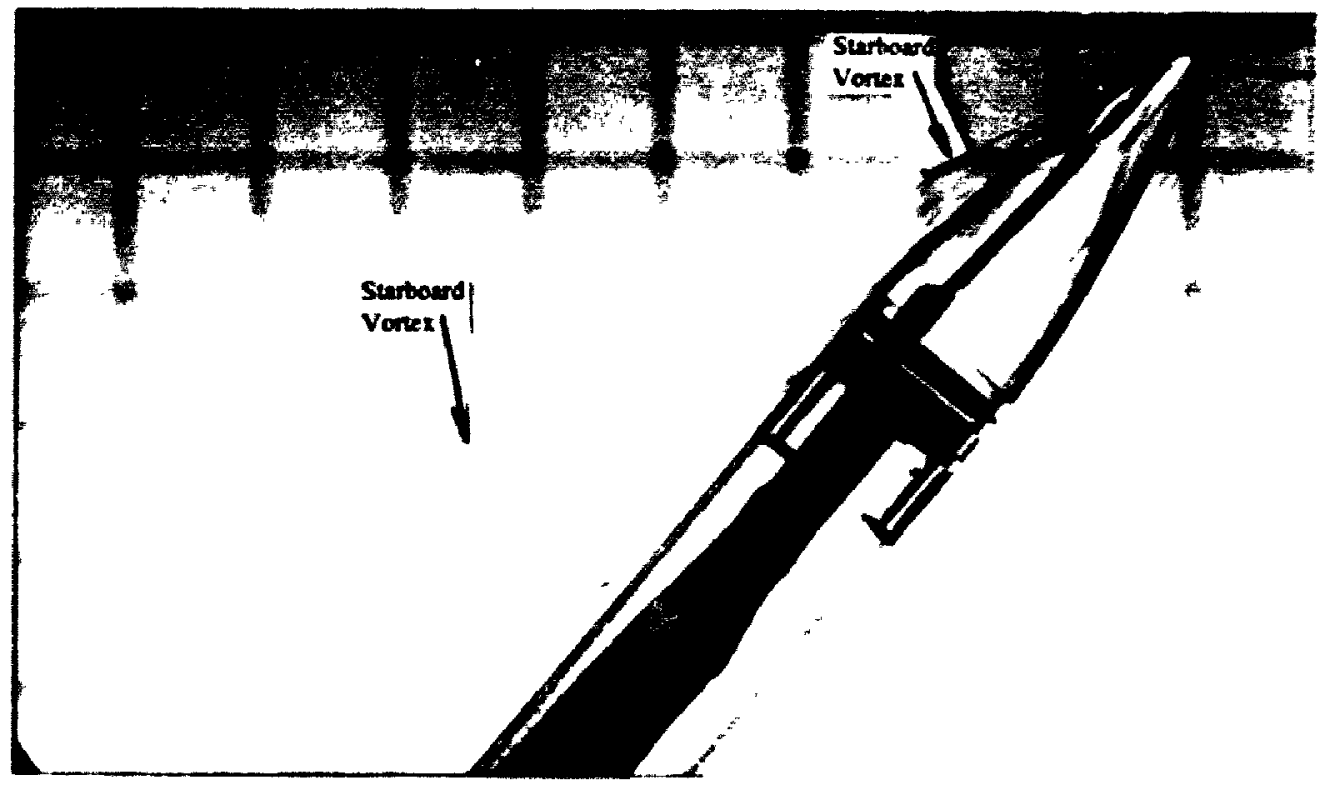

(b) Side view:

Figure 4.6 Flow visualisation of the baseline flowfield $\alpha=50^{\circ}, \beta=0^{\circ}, R_{1}=1900, C_{2}=0^{\circ}$ 


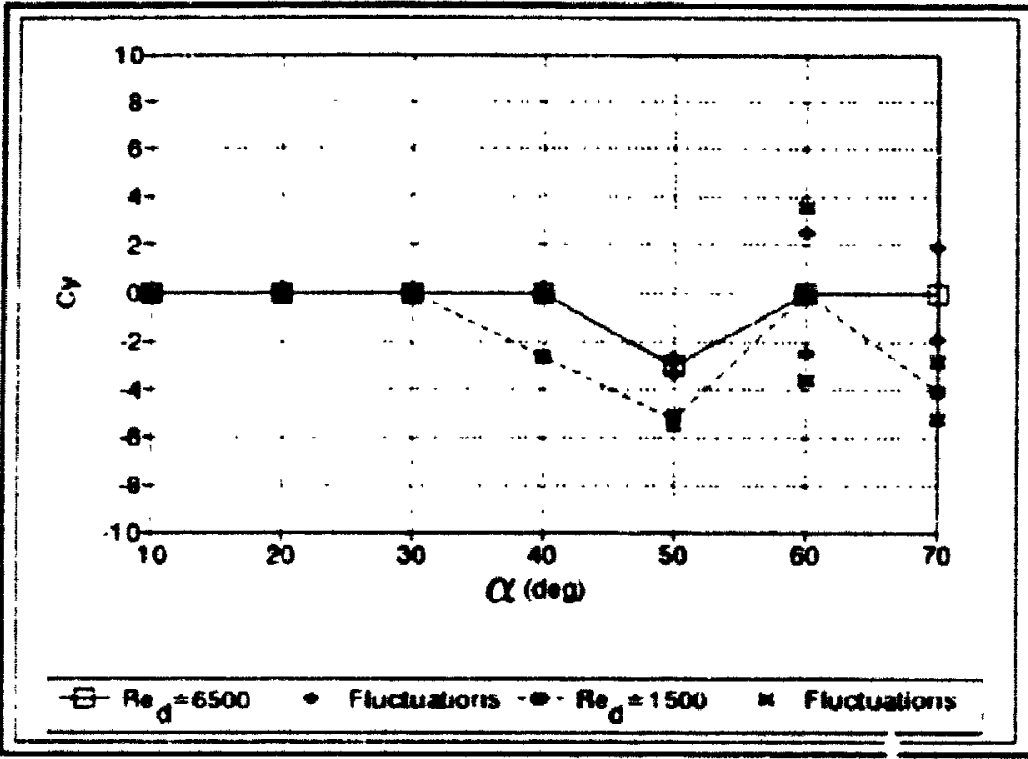

Fiqure $\mathbf{4 . 7 ( a )}$ Baseline flowfield sensitivity to Reynolds number. Side force versus angle of attack. $B=0^{\circ}, C_{\alpha}=0$.

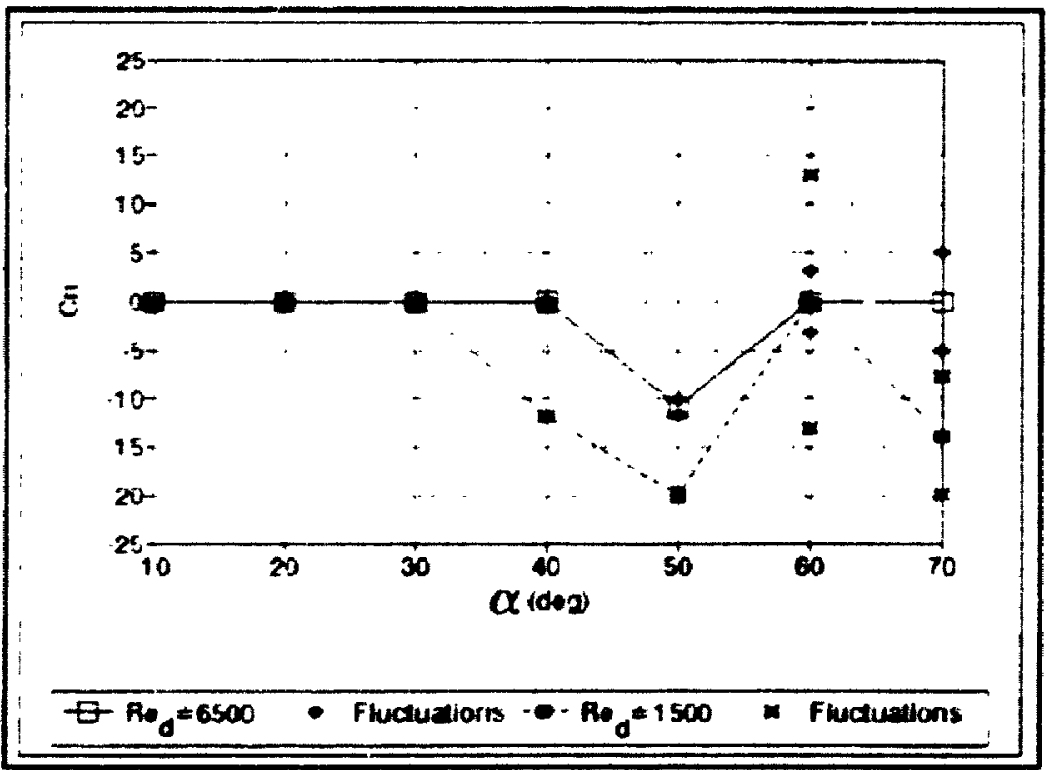

Figure 4.7(b) Baseline flowfield sensitivity to Reynolds number. Yaw moment versus angle of attack. $B=0^{n}, C_{*}=0$. 


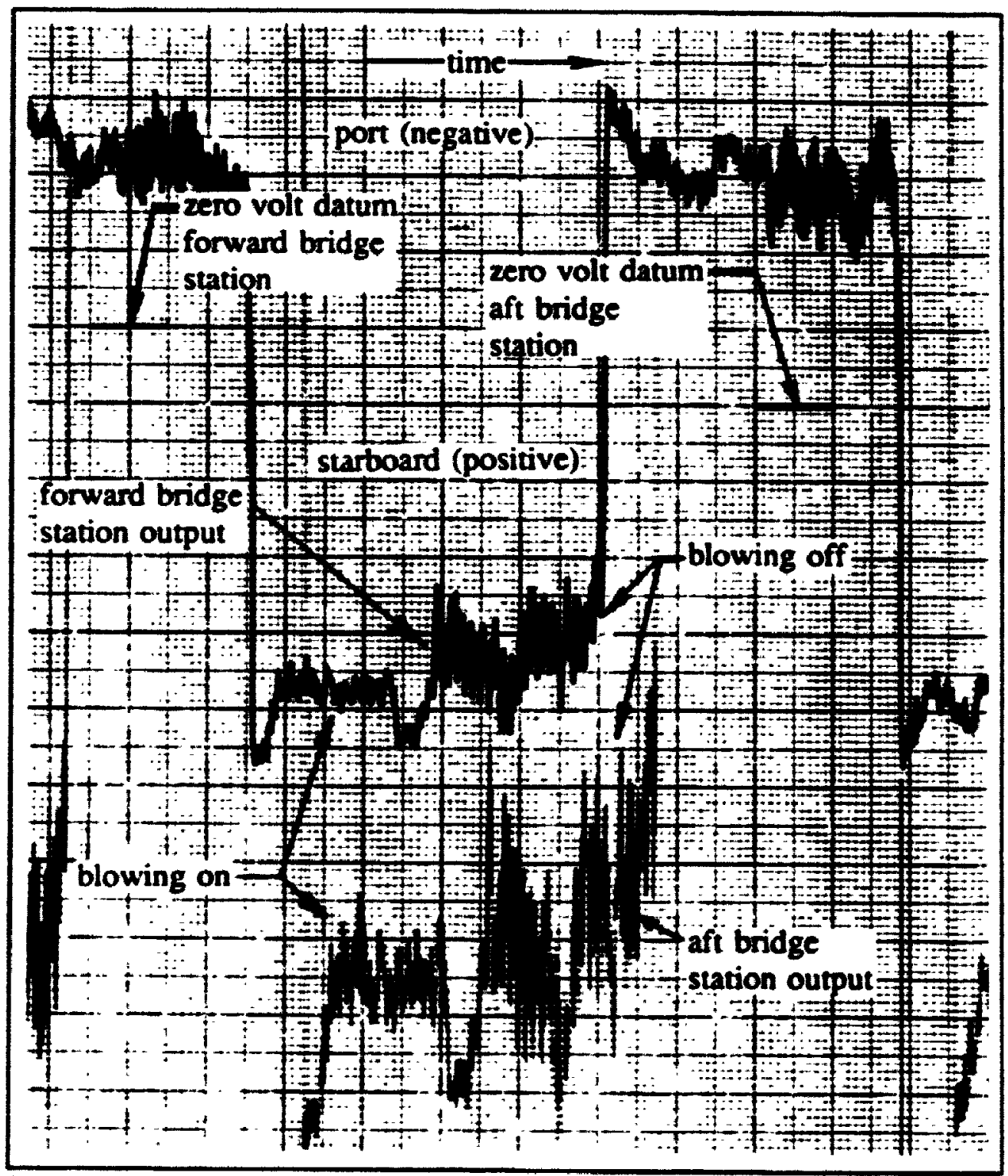

Figure 4.8 Stabilising effect of blowing. $\alpha=50^{\circ}, \beta=0^{\circ}, R e_{d}=7200, C_{c}= \pm 0.0013, \bar{\omega}=0$, censitivity: $0.04 \mathrm{~V} / \mathrm{cm}$, chart speed: $2.5 \mathrm{~cm} / \mathrm{min}$. 


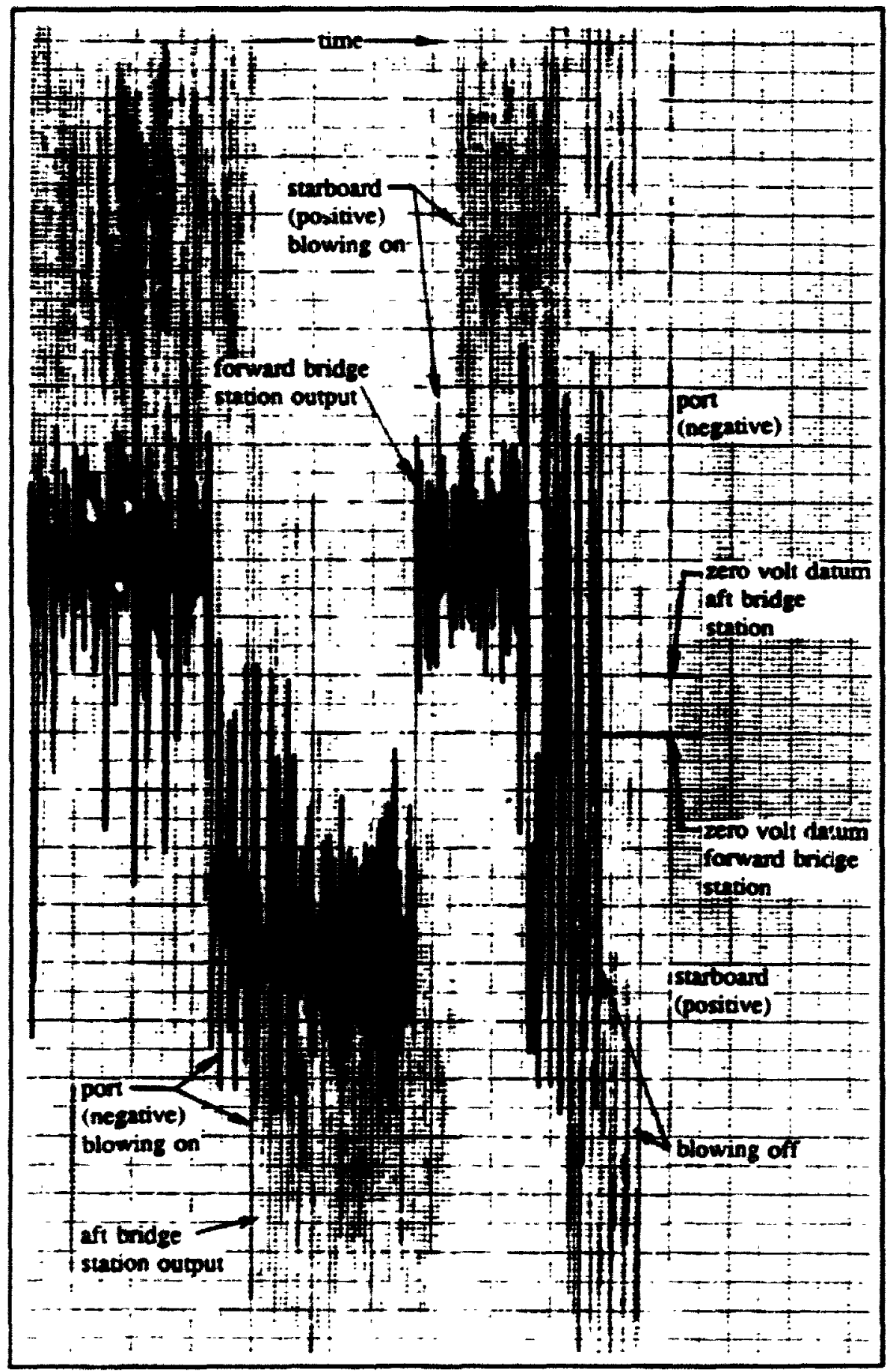

Figure 4.9 Control and stabilising effects of blowing. $\alpha=60^{\circ}, \beta=0^{\circ}, R_{e_{d}}=7200$, $C_{.}= \pm 0.0013, \bar{\omega} \approx 0$, seasituvity: $0.04 \mathrm{~V} / \mathrm{cm}$, chart speed: $2.5 \mathrm{~cm} / \mathrm{min}$. 


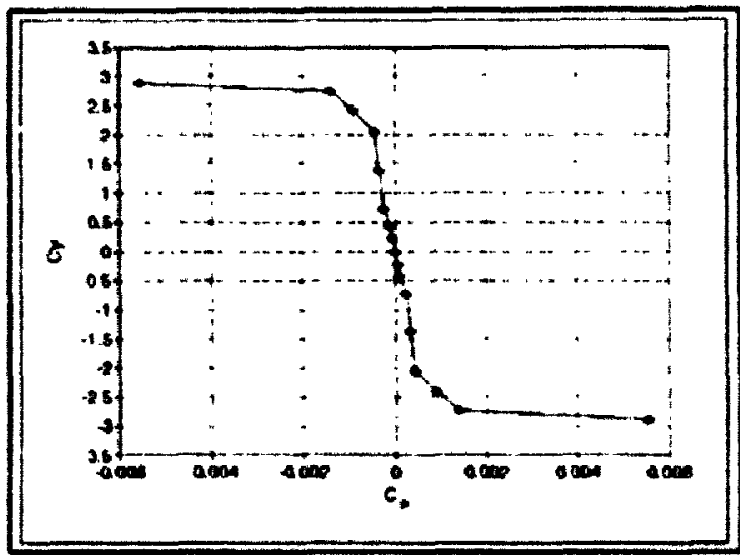

Figure 4.10(a) Mean side force versus coefficient of momentum blowing for the optimised configuration. $\alpha=50^{\circ}, \beta=0^{\circ}, \operatorname{Re}_{d}=6900, \bar{\omega}=0$.

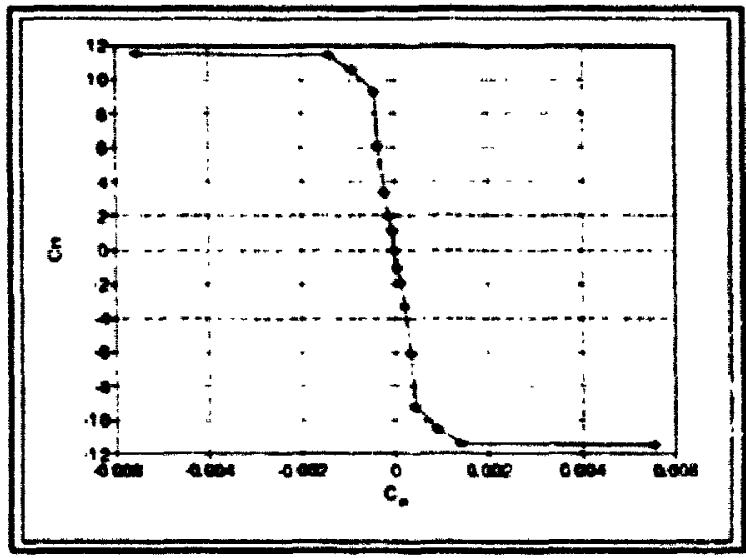

Figure 4.10(b) Mean yaw moment veraus coefficient of momentum blowing for the optimised configuration. $\alpha=50^{\circ}, \beta=0^{\circ}, \mathrm{Re}_{\mathrm{o}}=6900, \omega=0$.

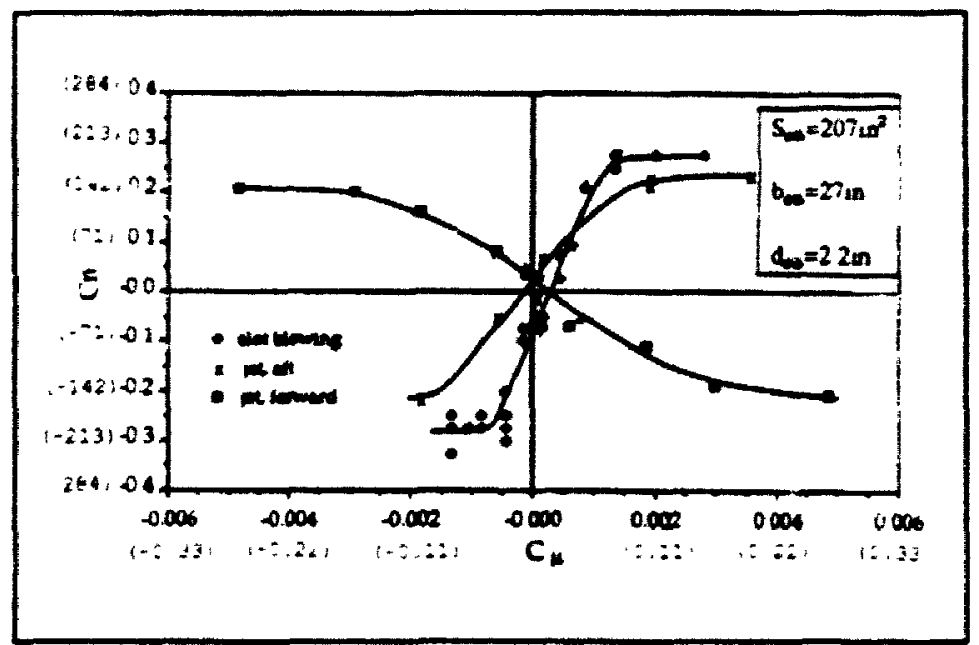

Figure 4.10(c) Steady-state mean yaw moment as a function of blowing for a $6 \%$ scale F/A-18 fighter aircraft model. Reproduced from figure 7 of Malcolm and $\mathrm{Ng}_{\mathrm{g}}$ (199/). 


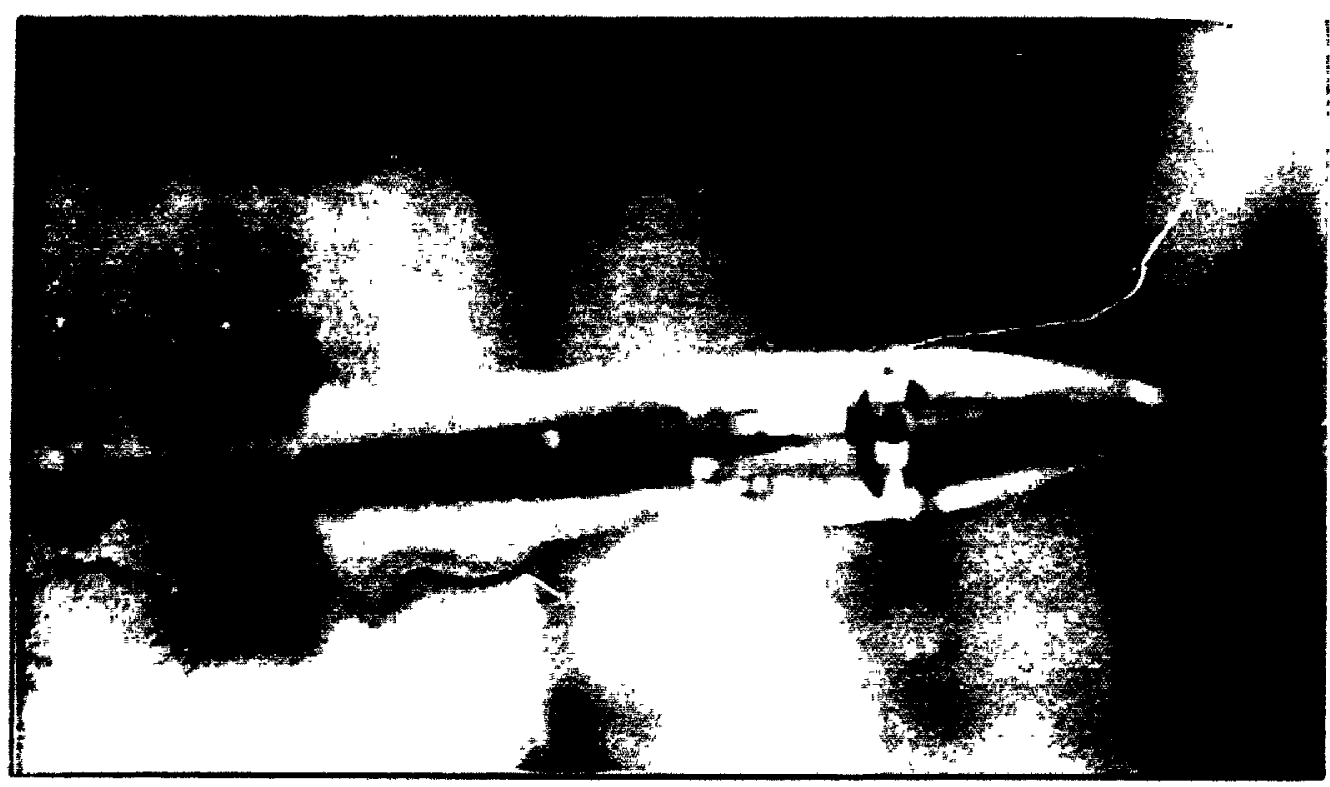

(a) Top view:

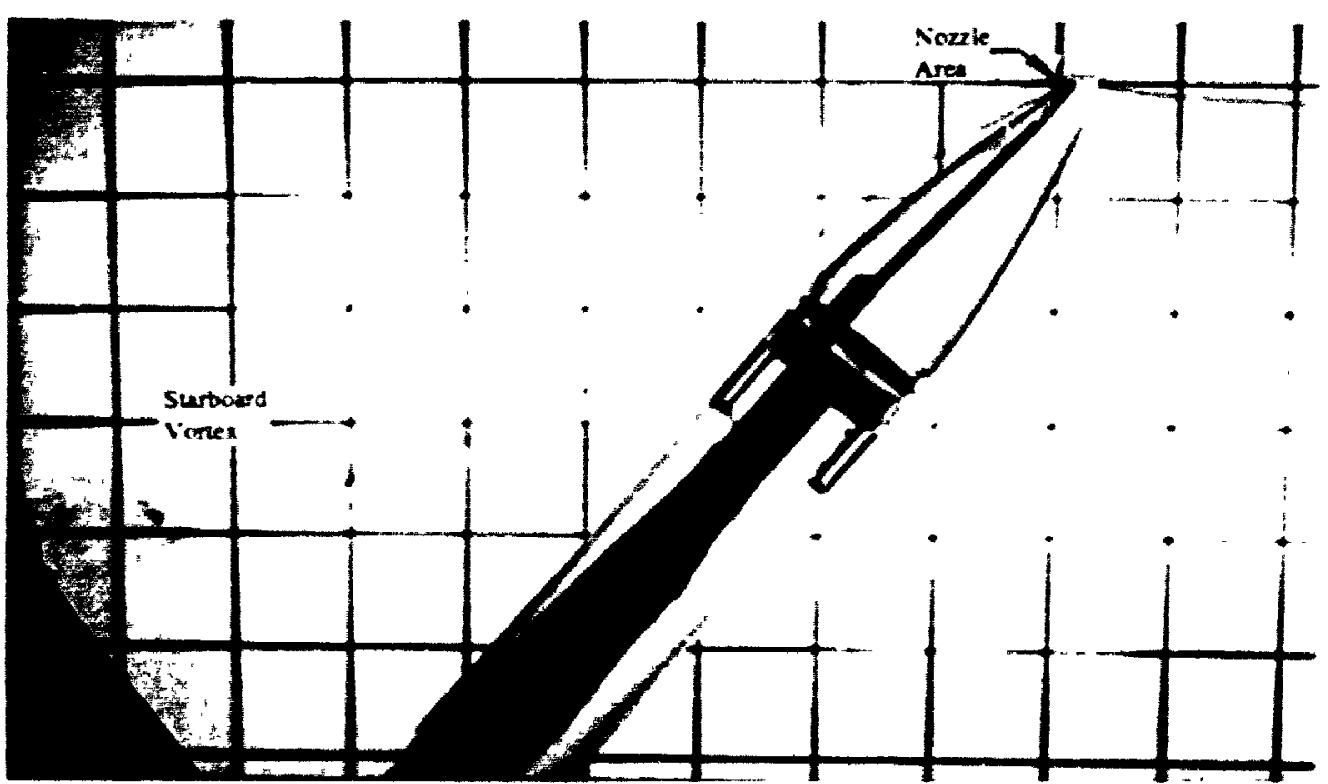

(b) Side view

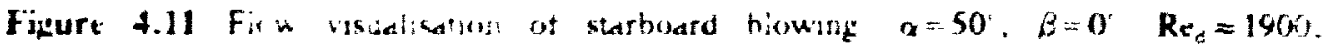
$C_{.}=-11 ;+2 ; 3,2=0$ 


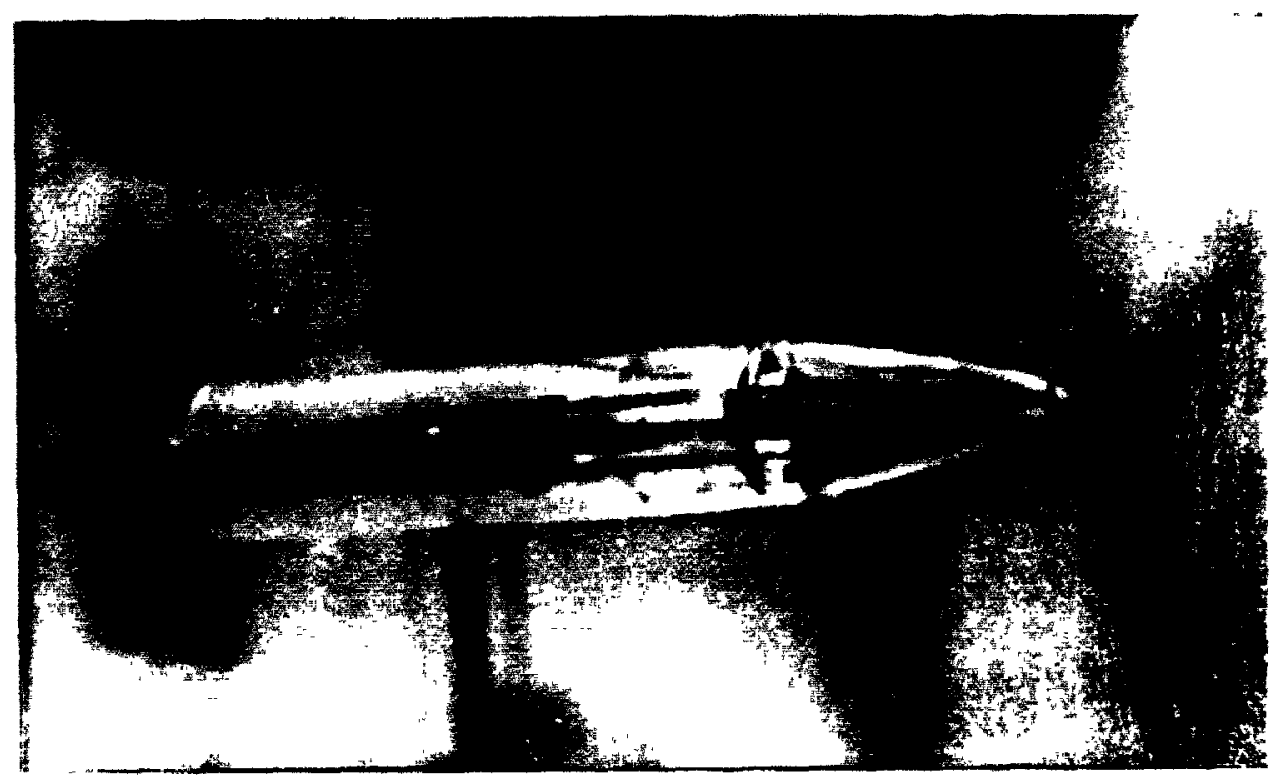

(a) Top vieu:

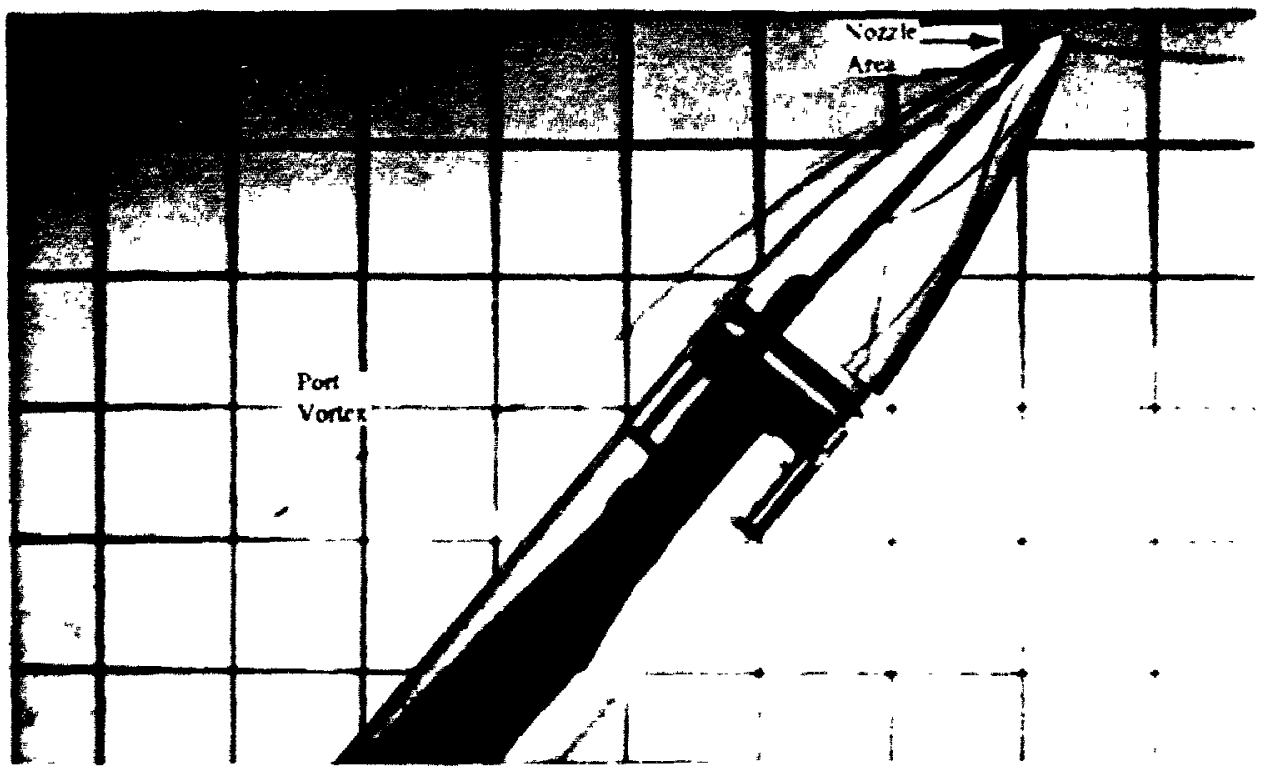

(b) Side vien

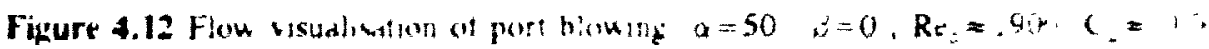
$a=0$. 


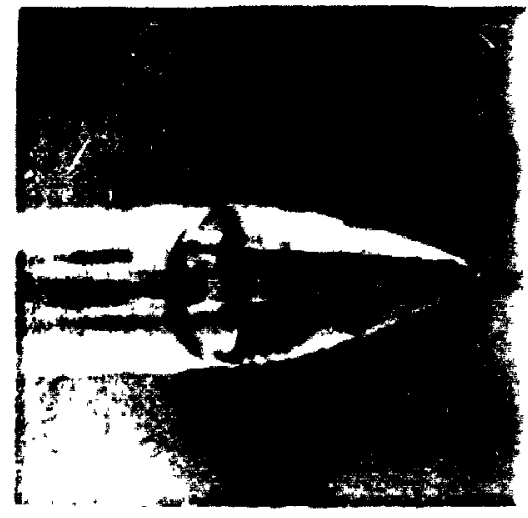

(a) Top ven.

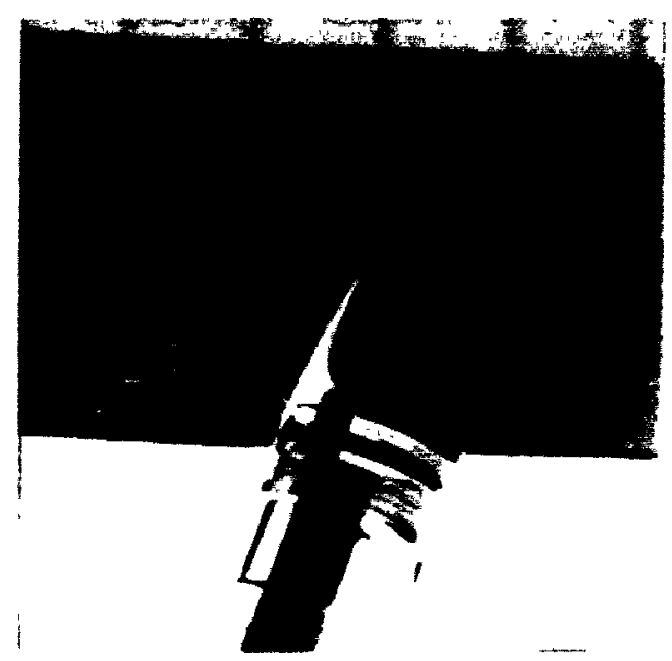

(b) Side vieu:

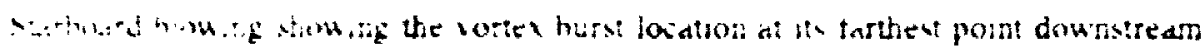
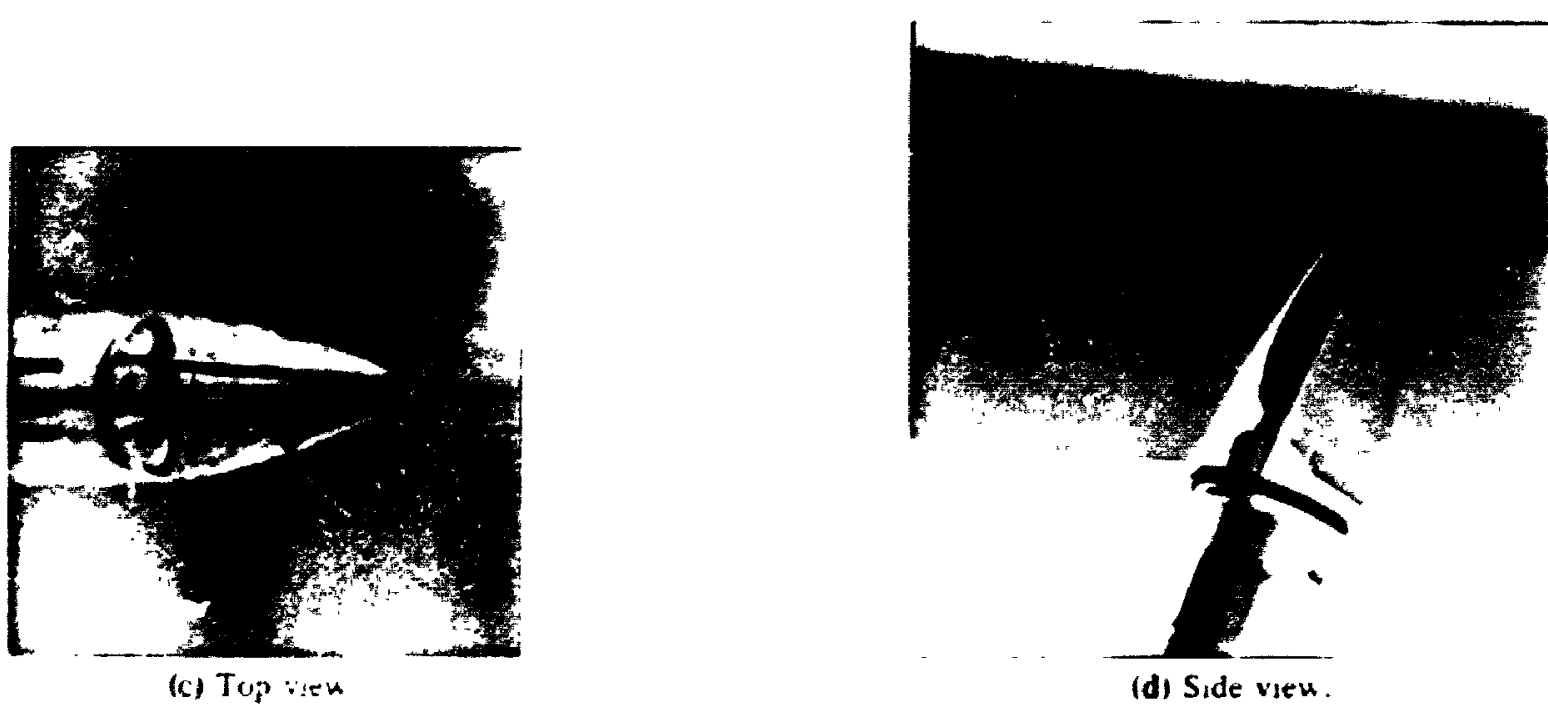

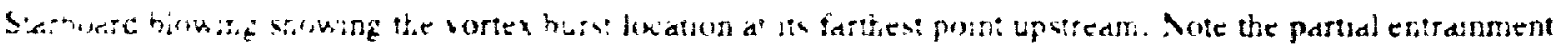

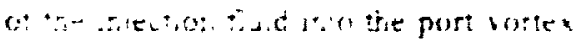

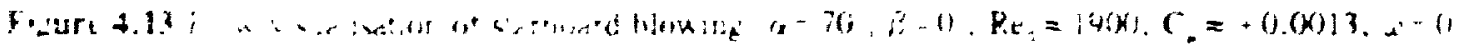




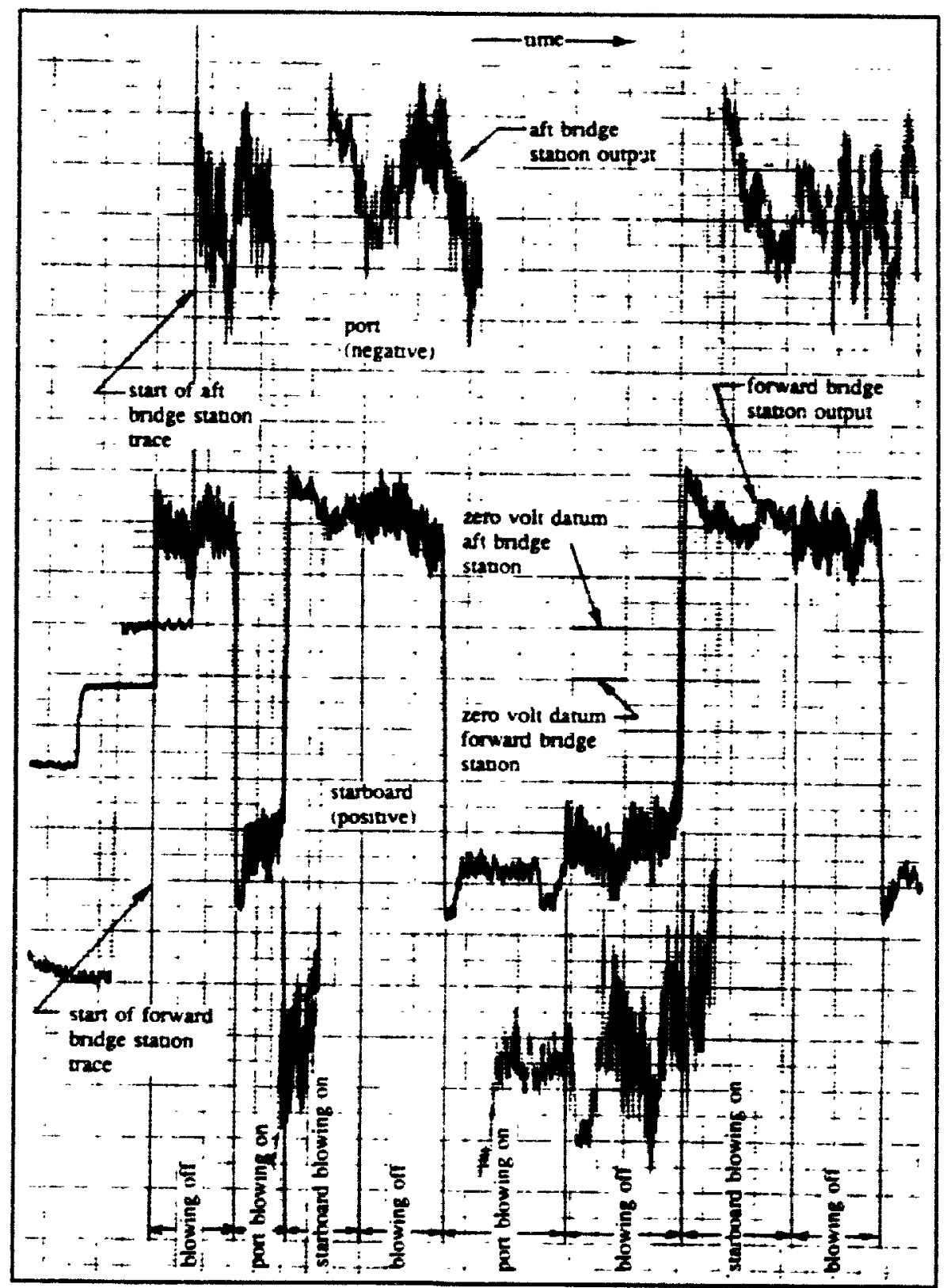

Figure 4.14 Forebody vortex control in the bi-stable flow regime. $\alpha=50^{\circ}, \beta=0^{\circ}$. $R_{e_{d}}=7200, C_{k} \approx \pm 0.0013, \bar{\omega} \approx 0$, sensitivity: $0.04 \mathrm{~V} / \mathrm{cm}$, chart speed: $2.5 \mathrm{~cm} / \mathrm{m} ı$. 

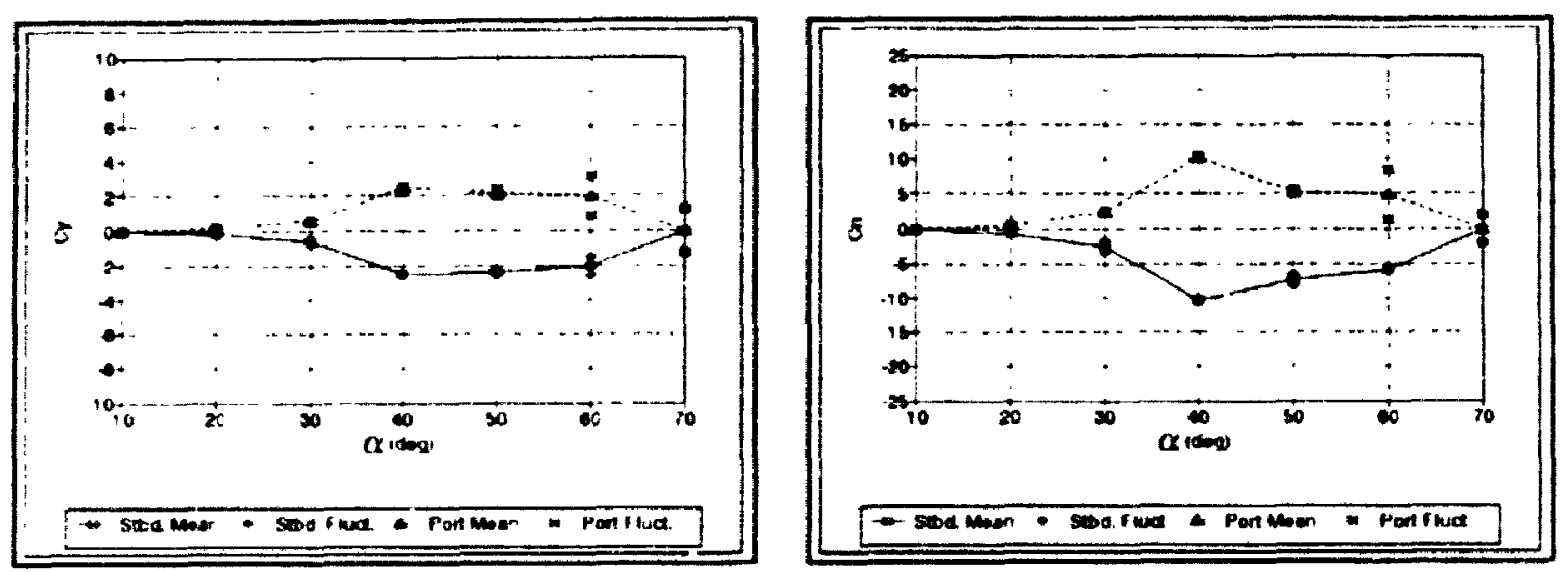

Figure 4.15(a) Individual port and starboard blowing. $\beta=0^{\circ}, R_{d}=7200, C_{n}= \pm 0.0013, \bar{\omega}=0$.
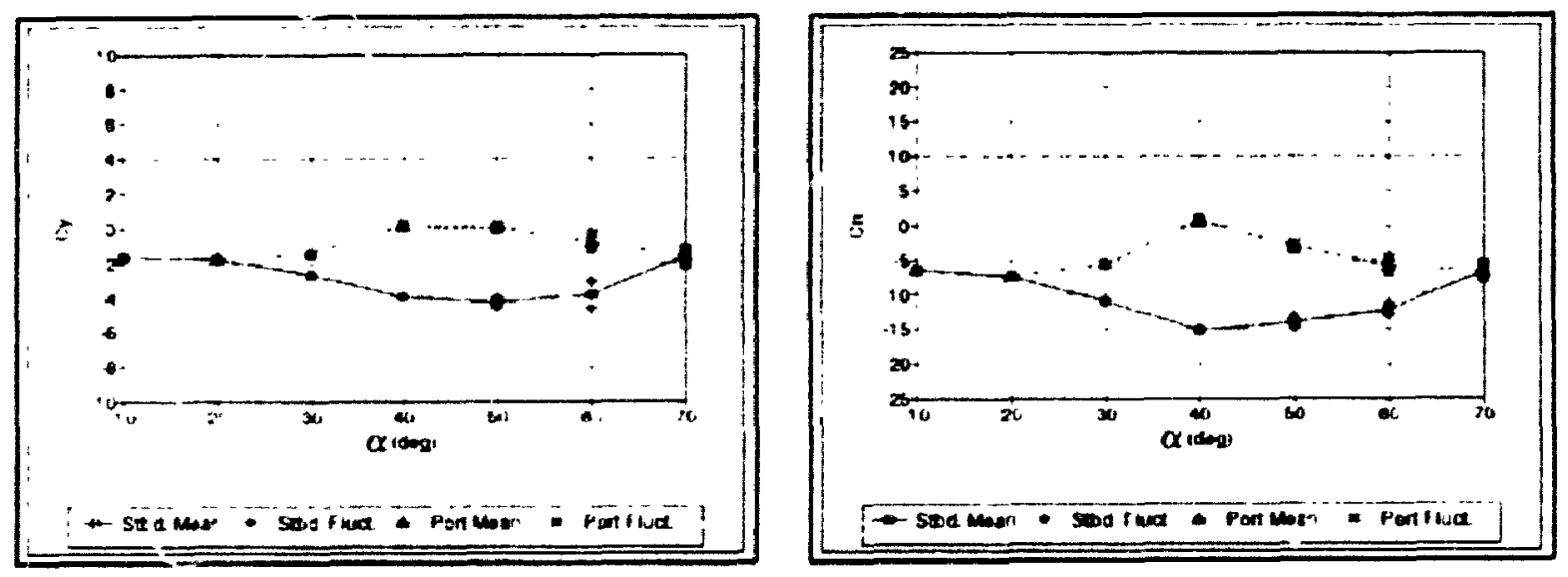

Fugure 4.15(b) Individual port and starboard blowing. $\beta=-10^{\circ}, \operatorname{Re}_{d}=7200, C_{-}= \pm 0.0013, \bar{\omega}=0$.
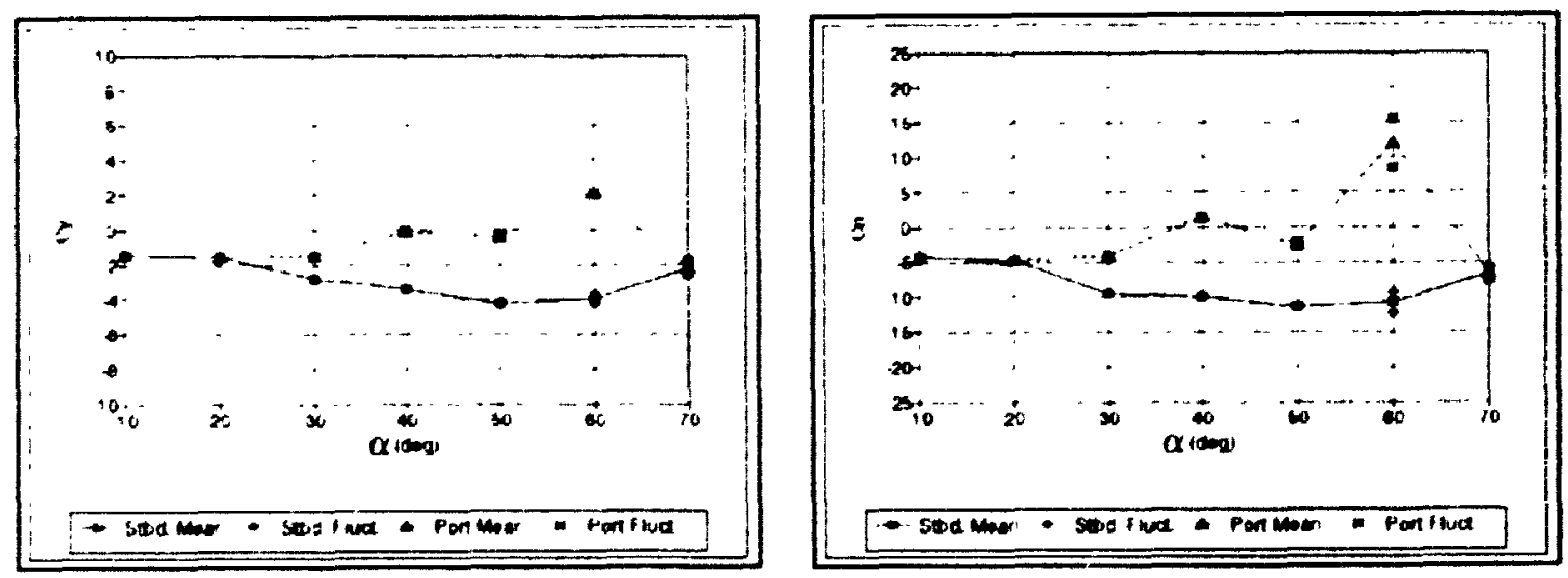

Figure 4.15(c) Individual port and starboard blowing. $\beta=-20^{\circ}, R_{d}=7200, C_{\alpha}= \pm 0.0013, \bar{\omega}=0$. 


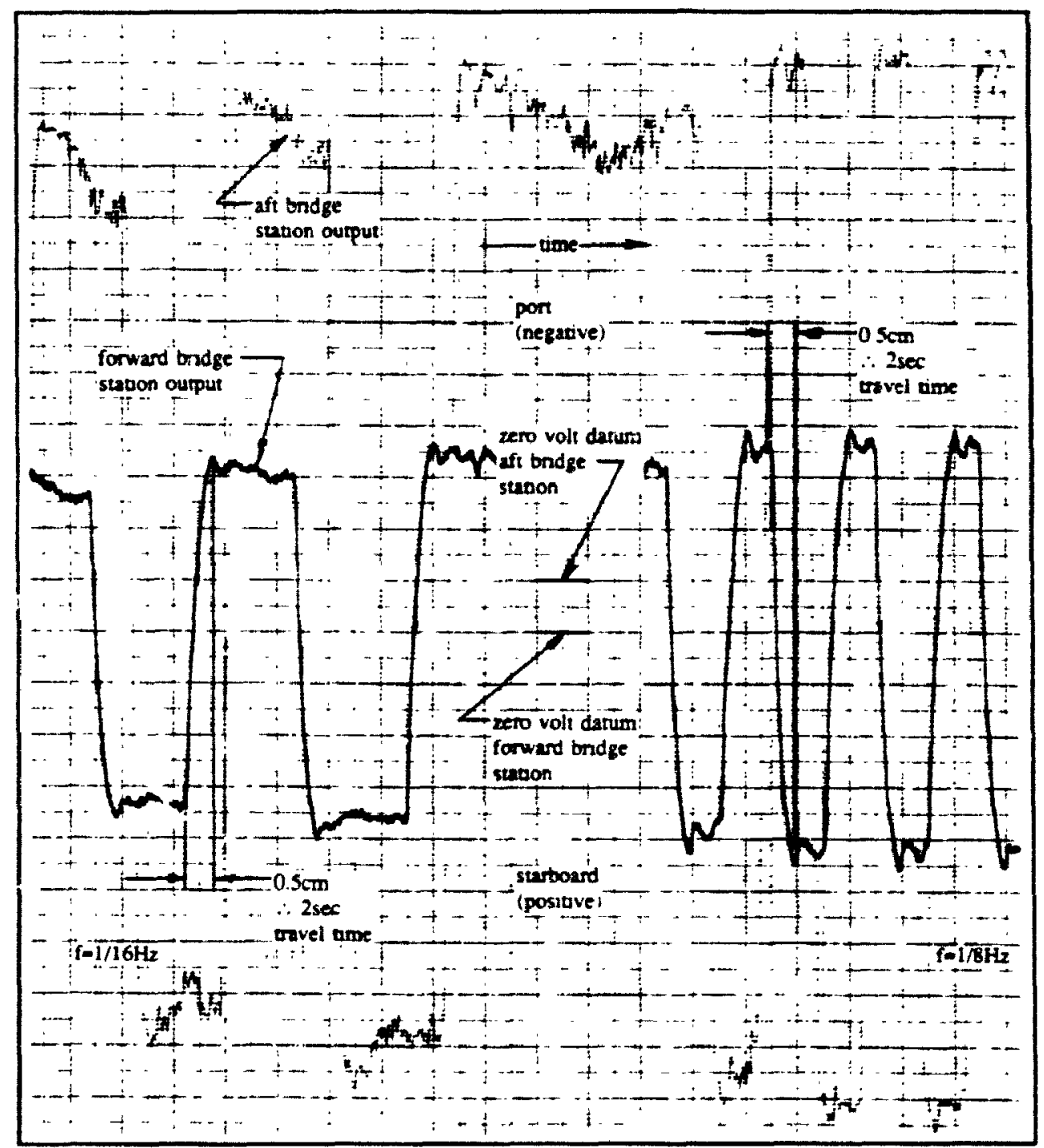

Fiqure 4.16 Estımation of maximum allowable frequency of oscillatory blowing $\alpha=50^{\circ}$. $\beta=0^{\circ}, R_{\mathrm{d}}=7200 . C_{n}= \pm 0.0013$. sensitivity: $0.04 \mathrm{~V} / \mathrm{cm}$, chart speed: $15 \mathrm{~cm} / \mathrm{min}$. 


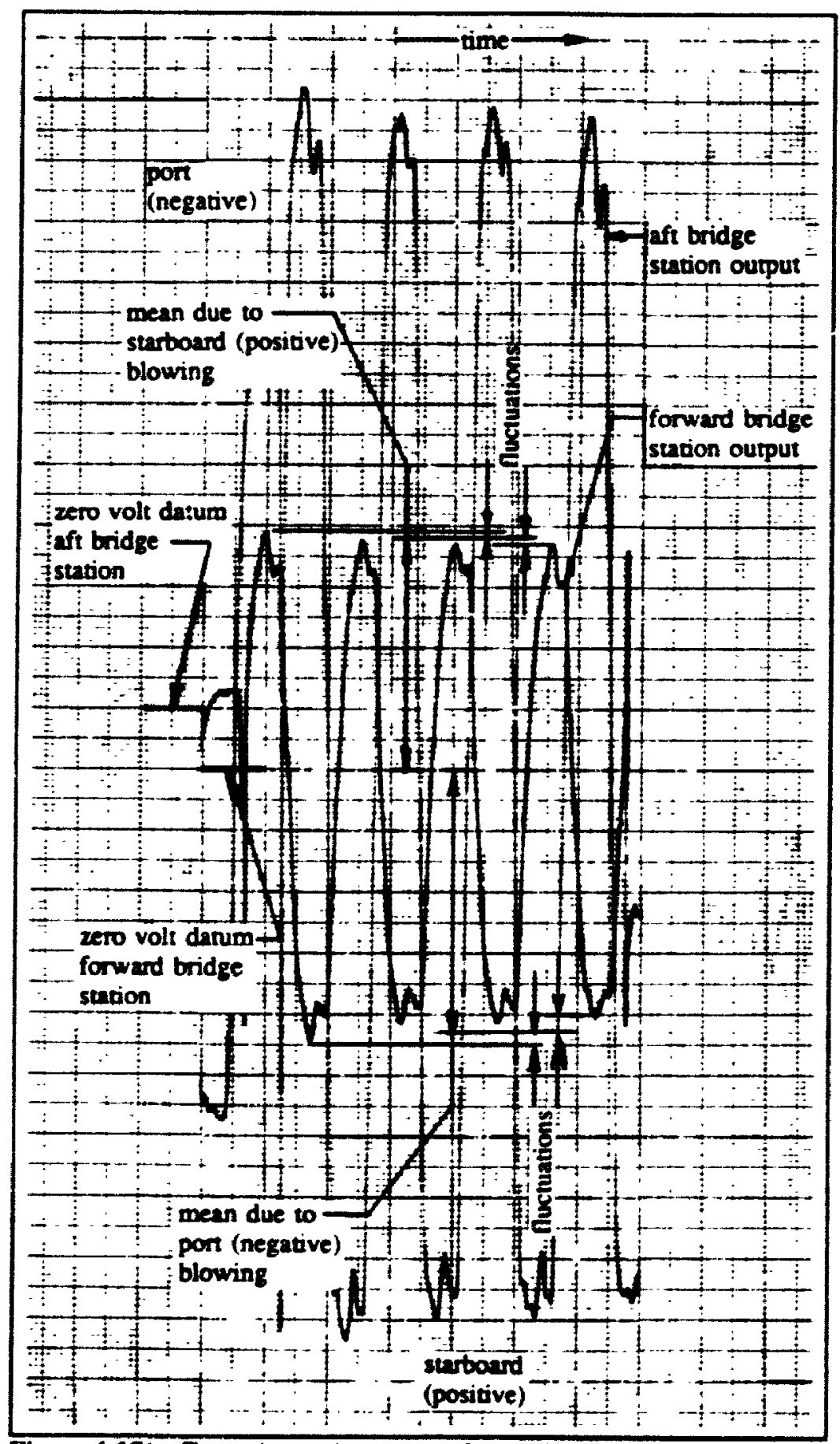

Fiqure 4.17(a) Typical stripchart output for oscillatory blowing below the maximum allowable frequency. $\alpha=50^{\circ}, \beta=0^{\circ}, R_{\alpha}=7100, C_{\alpha}= \pm 0.0013$. $\bar{\omega} \approx 0.08$. sensitivity: $0.04 \mathrm{~V} / \mathrm{cm}$, chart speed: $15 \mathrm{~cm} / \mathrm{min}$. 


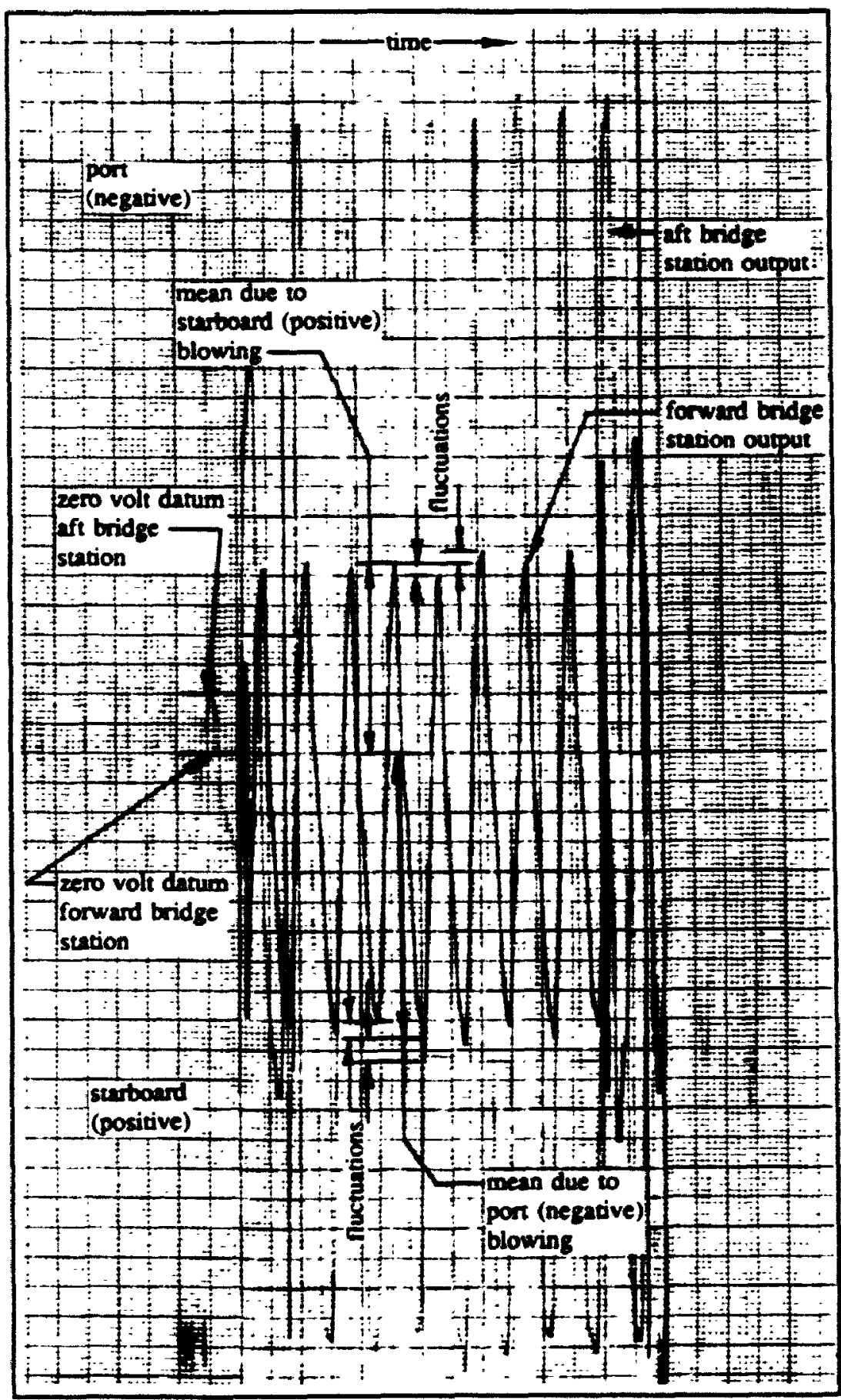

Figure 4.17(b) Typical stripchan output for oscillatory blowing at the maximum: allowable frequency. $\alpha=50^{\circ}, \beta=0^{\circ}, R_{d}=7400, C_{*}= \pm 0.0012$. $\bar{\omega}=0.16$, sensituvity: $0.04 \mathrm{~V} / \mathrm{cm}$, chart opeed: $15 \mathrm{~cm} / \mathrm{min}$. 

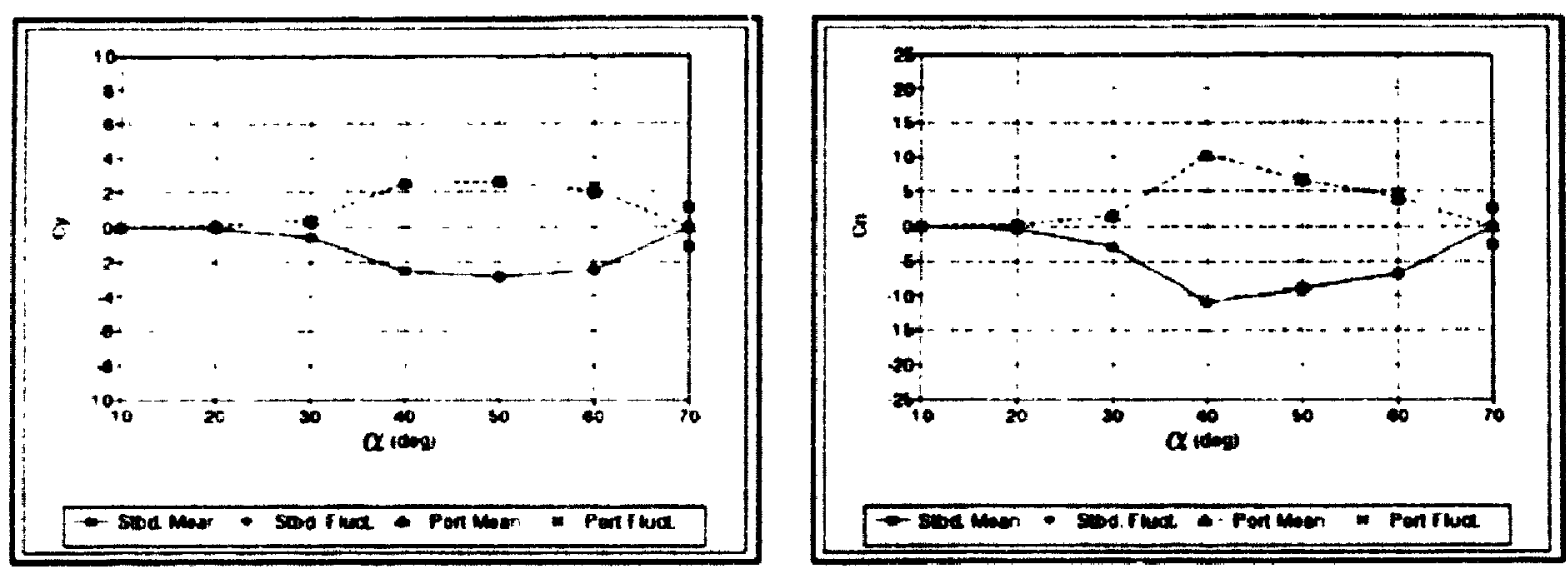

Figure 4.18(a) Port and starboard oscillatory blowing. $\beta=\sigma^{\prime}, R e_{s}=7100, C_{\alpha}= \pm 0.0013, \bar{\omega}=0.08$.
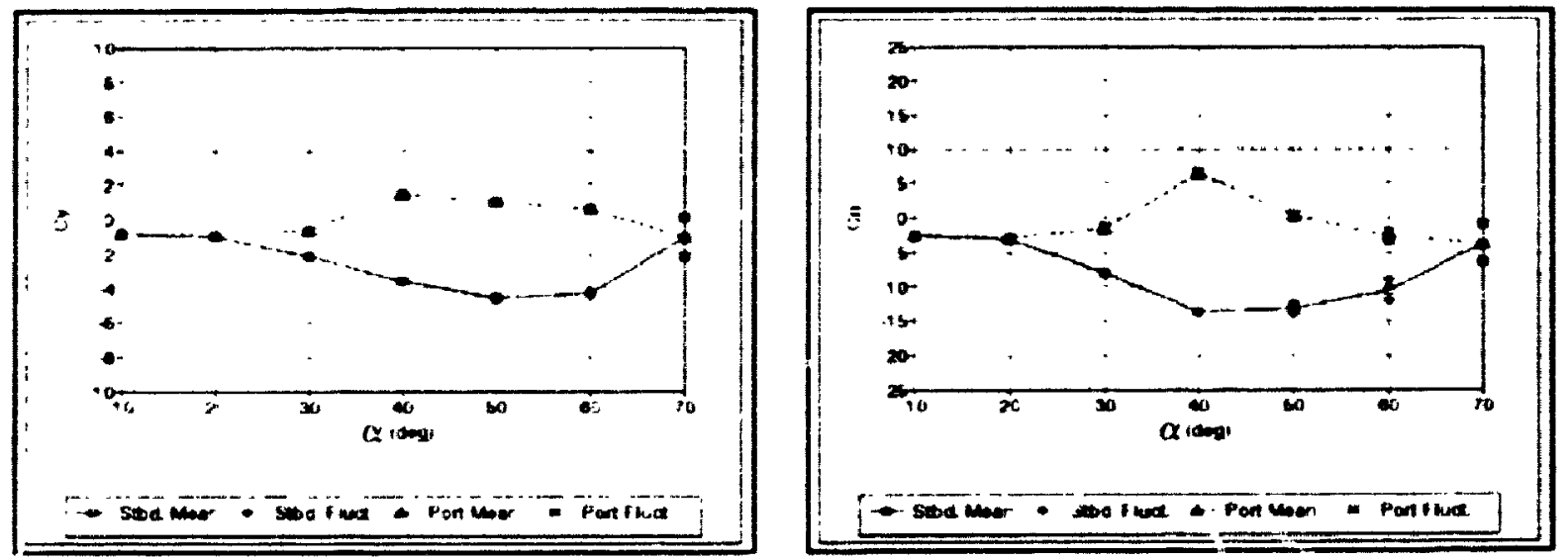

Figure 4.18(b) Port and starboard oschllatory blowing. $B=-10^{\circ}, R_{d}=7100, C_{\alpha}= \pm 0.0213, \bar{c}=0.08$.
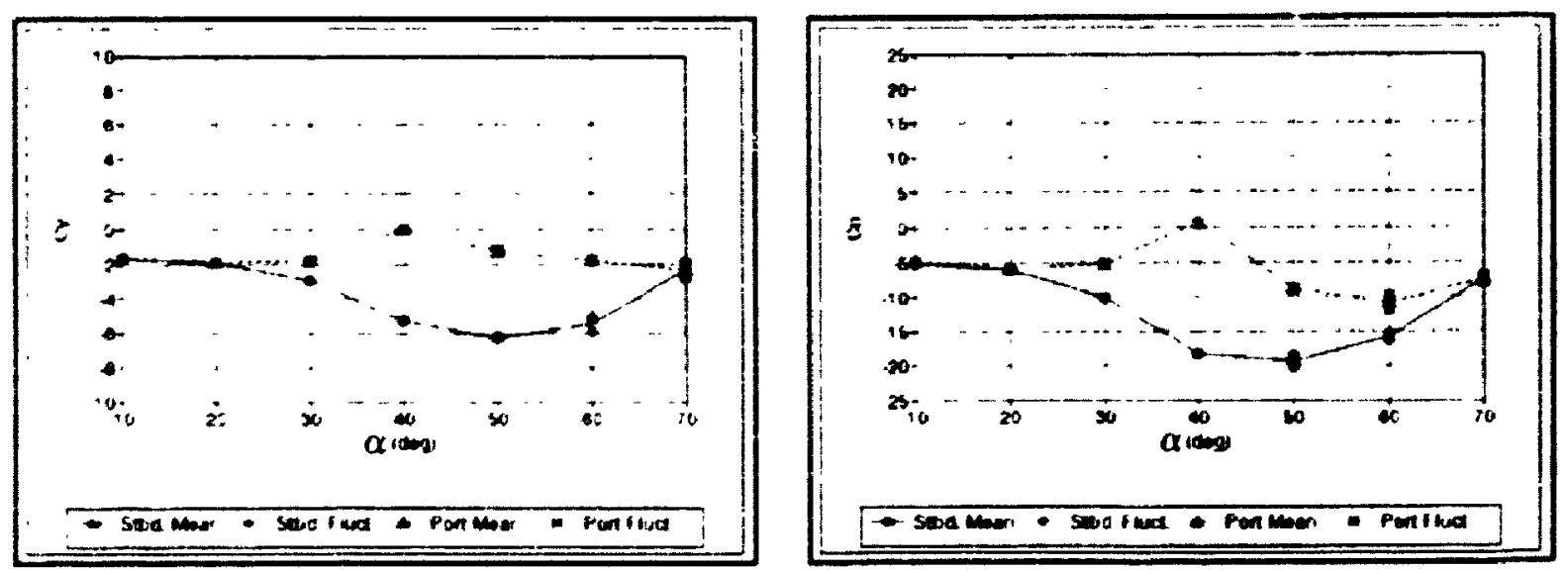

Figure 4.18(c) Port and starboard oscillatory blowing. $B=-20^{\circ}, R e_{d}=7100, C_{*}= \pm 0.0013, \bar{\omega}=0.08$. 

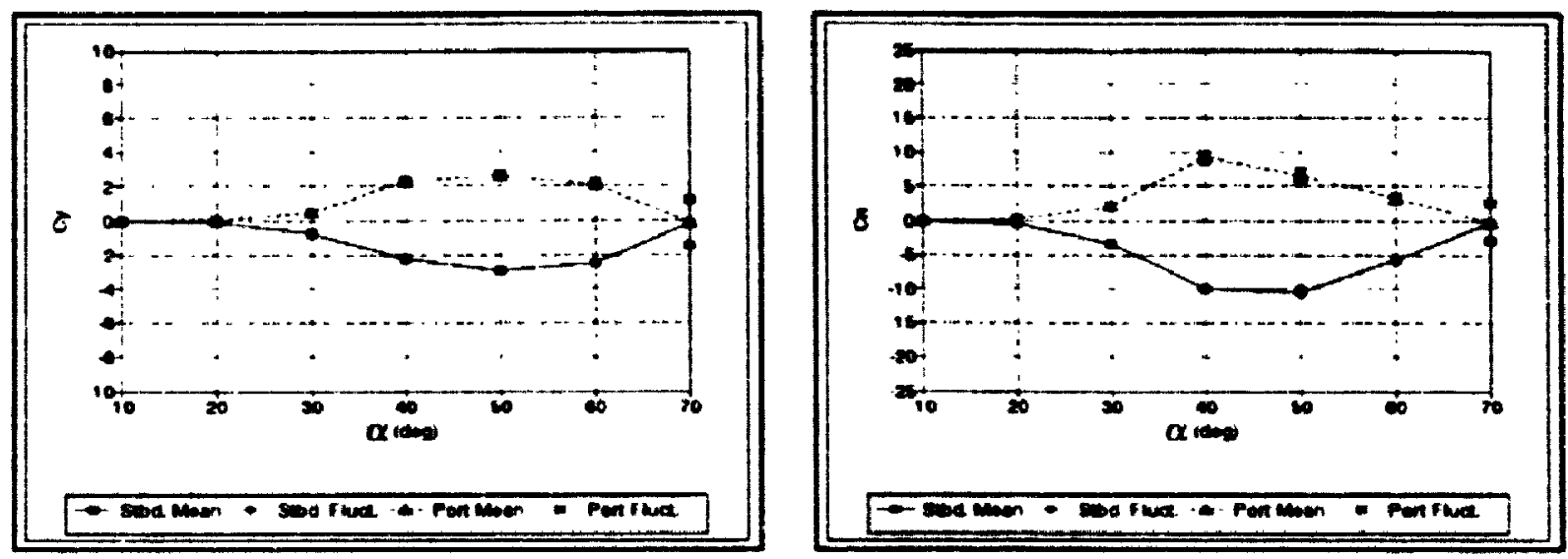

Figure 4.19(a) Port and starboard oscillatory blowing. $\beta=0^{\circ}, R e_{d}=7400, C_{s}= \pm 0.0012, \bar{v}=0.16$.
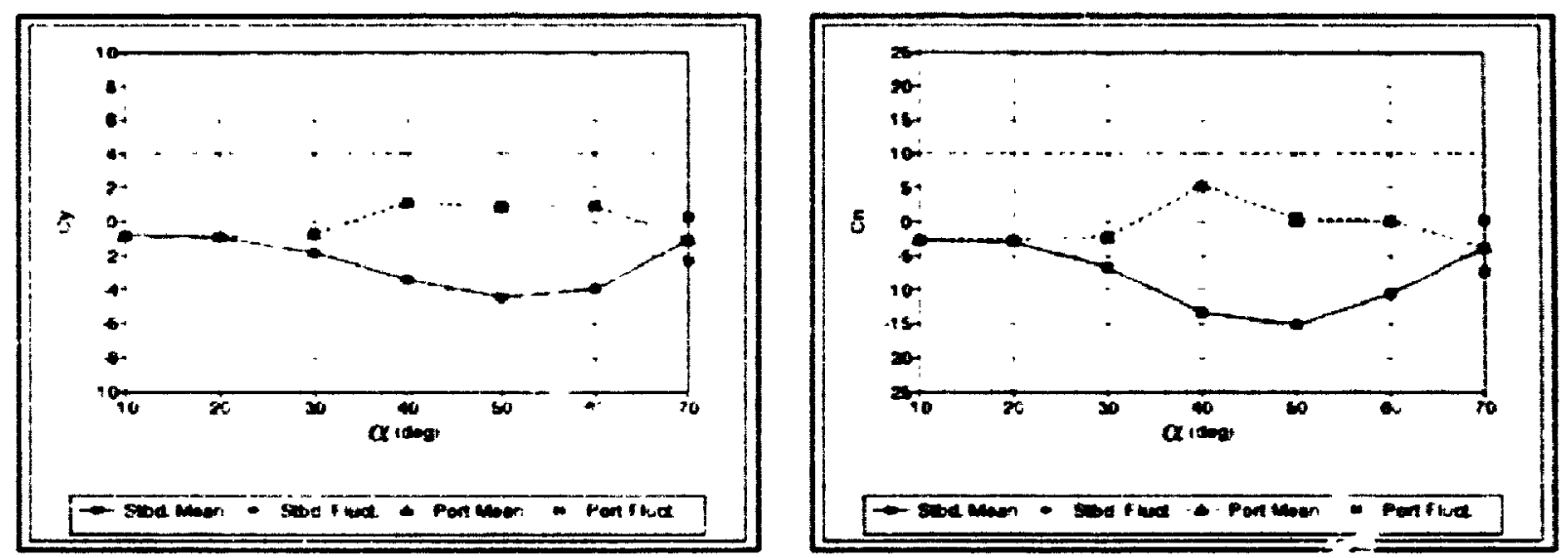

Figure 4.19(b) Port and starboard oscillatory blowing. $B=-10^{\circ}, \operatorname{Re}_{d}=7400, C_{*}= \pm 0.0012, \bar{z}=0.16$.
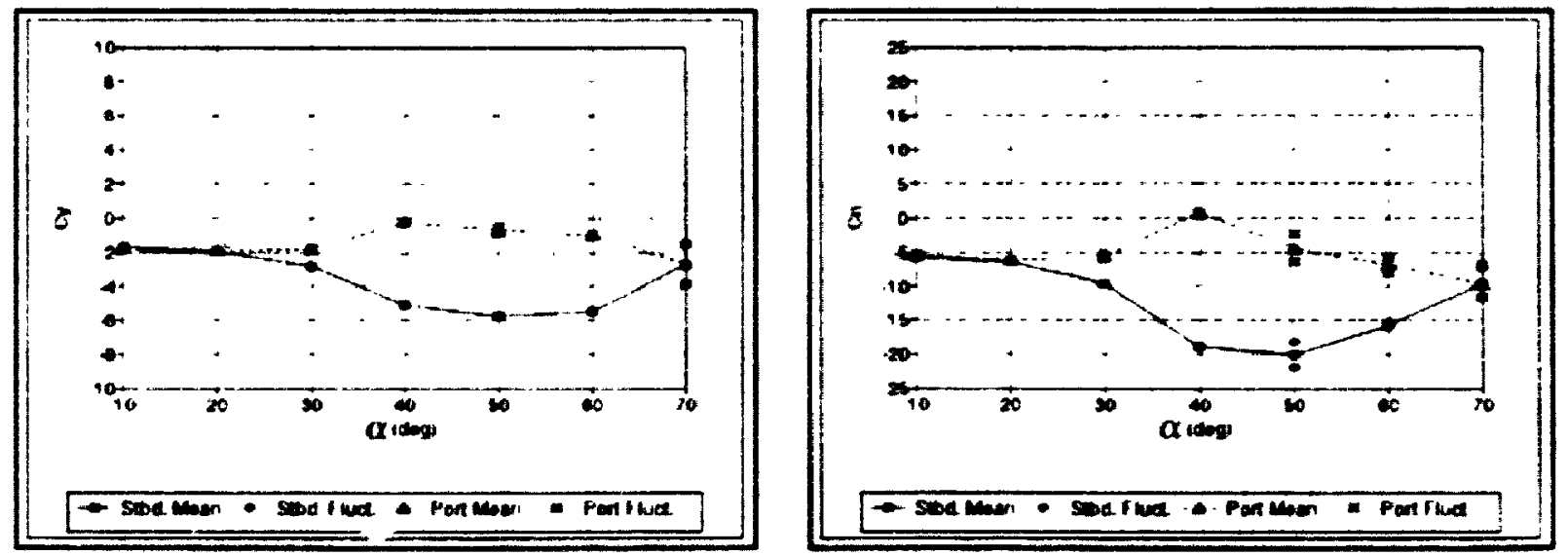

Figure 4.19(c) Port and starboard oscillatory blowing. $\beta=+20^{\circ}, \operatorname{Re}_{d}=7400, C_{\alpha}= \pm 0.0012, \bar{w}=0.16$ 


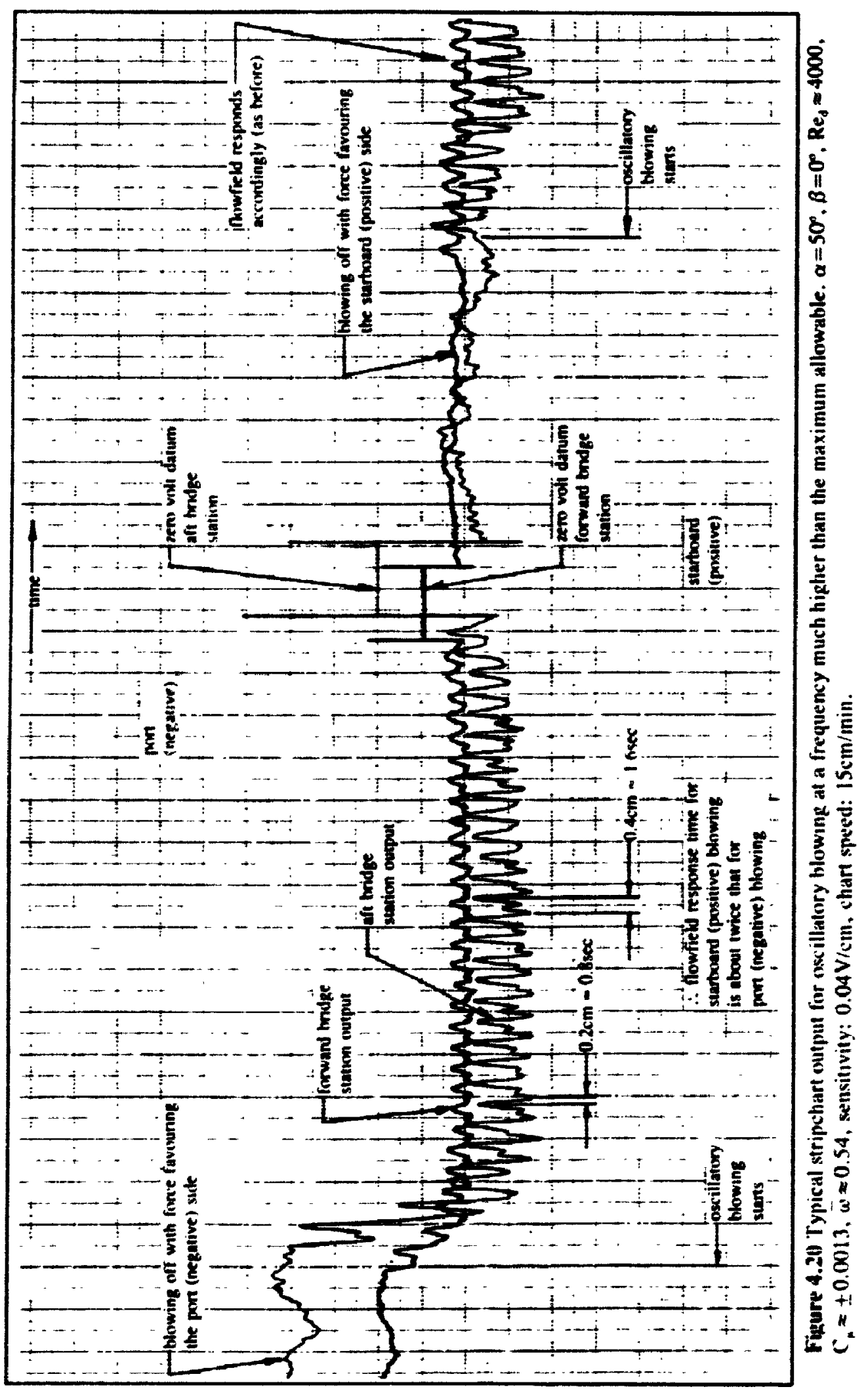




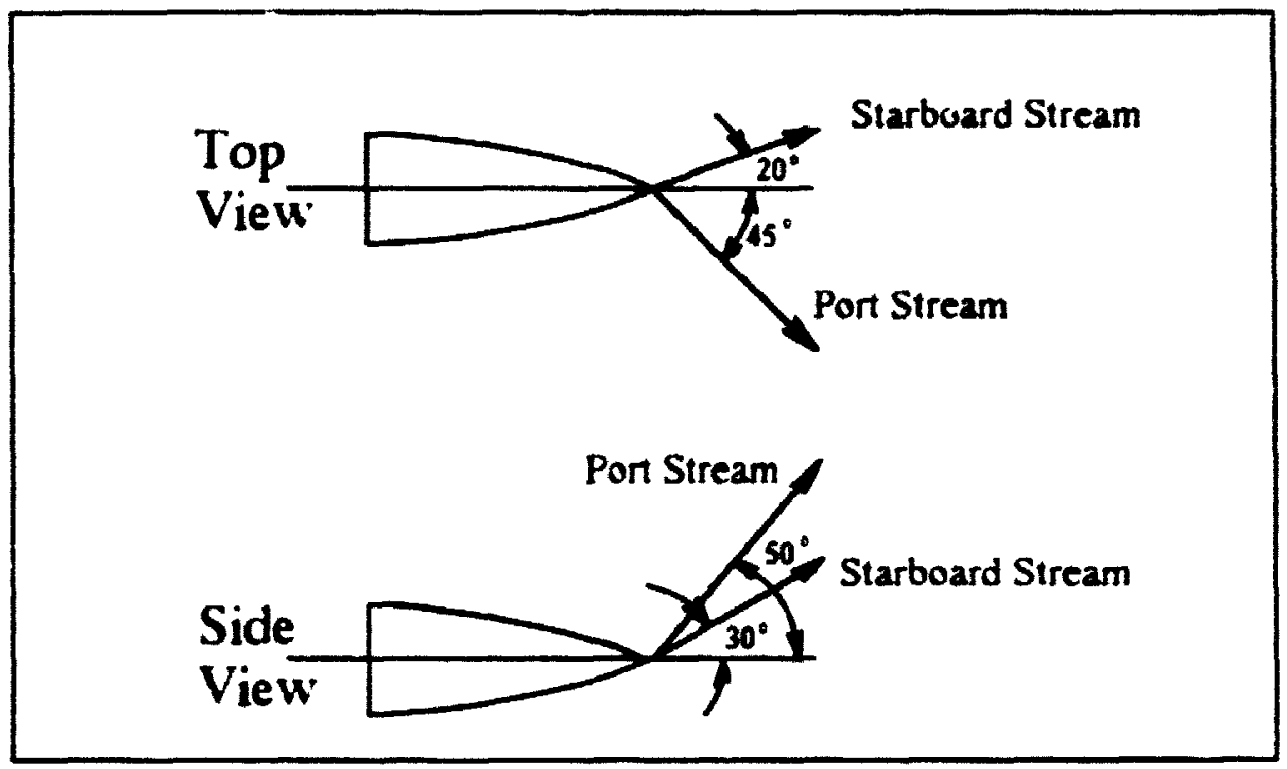

Fagure 4.21 Nozzle jet exut stream trajectones. Note: Angles shown are approximate. 


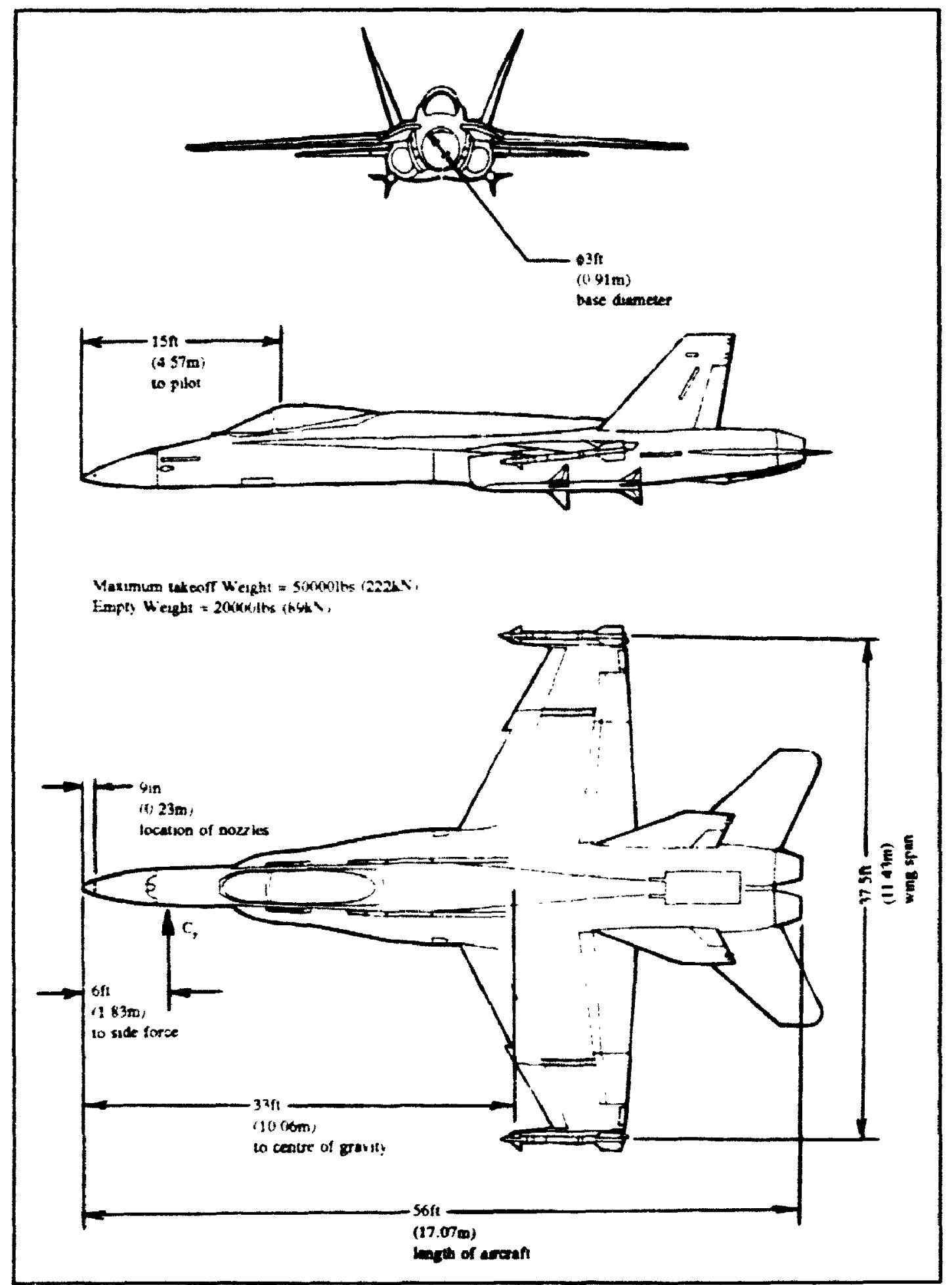

Fizure 4.22 Selected F/A-18 fighter arcraft specifications. 
Appendix A: Equipment Lists and Specifications 


\section{Swimming Pool Chlorine}

The chemical used to clean the uater tunnel and maintain clarity of the water was calcium hypochlorite. It is a dry granular chlorine called Chlonve. manufactured by:

Saskatoon Chemicals

P.O. Box 1586

Saskatoon. Saskatchewan

S7K $3 B 3$.

\section{List of Experimental Equipment}

Strain Gauges: Micro-Measurements Group general purpose foil strain gauges with a resistance of $120 \mathrm{Ohms}$, a gauge factor of 2.12 , and a temperature sensitivity of \pm 2 microstrains per degree Celsius.

Stripchant Recorder. Heulett Packard HP 7132A, 2 channel stripchant recorder.

Strain Indicators: Vishay Measurement Group P-350A portable strain indicators. Forward bridge S/N 009851. aft bridge S/N 043655.

Needle Valves: $\quad$ Nupro M-Series B-4MG2 metering valves.

Fiou Meters: Duyer RMA-32 rotameters.

Solenoid Valve: Asco 1 4-8320 B176P 3-way. 2 position universal brass solenoid valie.

\section{Waterproofing Compounds}

The compounds used to uaterproof the strain gauges and their tead wires were manufactured by Micro-Measurements Group and distributed by Intertechnology. They are described belon. 
M-COAT-B: $\quad$ Nitrile rubber compound used to coat the exposed vinyl coated leadwires.

M-COAT-A: $\quad$ Polyurethane used as the first layer of protection over the strain gauges.

M-COAT-W1: $\quad$ Microcrystalline uax used as the second layer of protection over the strain gauges.

Ttitun Film: Intended as an undercoatung for the polysulphide rubber compound. this adhesive teflon sheet was used as the third layer of protection over the strain gauges.

M-COAT-J: $\quad$ Polvulphide rubber ciningunid used as the fourth and fifth lavers of protection over the strain gauges. 
Appendix B: Sting and Balance Characteristics 


\section{Temperature Sensitivity}

The strain gauge bridges were not temperature compensated, however this did not have a significant effect on the data because the water was allowed to enter a state of thermal equilibrium with the room prior to testing. Since the water tunnel contained a relatively large volume of water, temperature changes during a test run were sma::.

\section{Lift and Side Force Cross-Coupling}

Application of the same forces in lift as those used to calibrate the balance in the side force-plane, as described in chapter 3. resulted in outputs from the strain gauges that were about 80 times smaller than those shown in figure 3.13. Consequently, the contribution of lift forces to the strain gauge bridge outputs was neglected.

\section{Sting Natural Frequency}

Subjecting the sting-model assembly to a momentary side force input resulted in a resonant frequency of about $16 \mathrm{~Hz}$ with a lightly damped rate of decay of oscillations. A graphical representation of the response is shown below. The logarithmic decrement was found to be about U. . . . . Bumping satio of approximately 0.02 [Thomson. 19811 This test was conducted in air. The natural frequency of the assembly in water would be somewhat less due to the added mass of the water. The frequency of oscillations of the side forces measured at $\alpha=70^{\circ}$ remained relatively constant throughout the course of these experiments. varying between 0.9 and $1.1 \mathrm{~Hz}$. It was also observed that no amouni of blowing, oscillatory or otherwise, had any effect on the flow field at 
this angle of attack. Hou ever, this behaviour is not likely caused by resonant oscillations since the frequency measured at $\alpha=70^{\circ}$ was about 16 times less than the natural frequency of the sting-model assembly in air. It is doubtful that the added mass caused by submerging the assembly in water would reduce its natural frequency by a factor of 16.

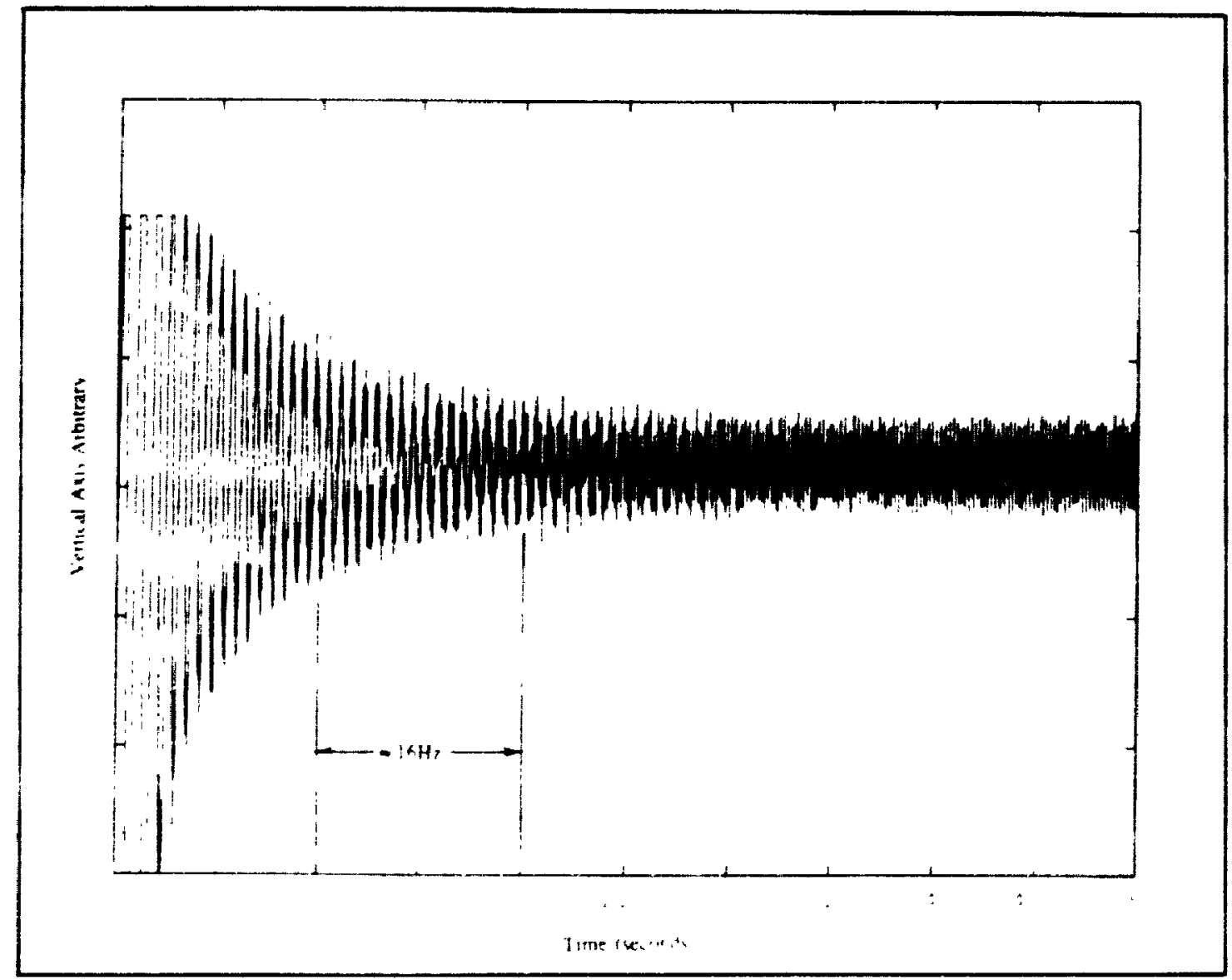

Frequency response of the sing-model assembly in air. 
Appendix C: Side Force and Yau Moment Equations 


\section{Side Force and Yaw Moment Equations}

Using the relations for bending moment and normal stress shown below [Beer and Johnston, 1981]:

$$
\sigma-\frac{M_{y}}{l}\left(\frac{t_{s}}{2}\right), \in-\frac{\sigma}{E}, M_{y}-\eta 7
$$

where: $\quad 0$ is normal stress.

$M_{y}$ is the bending moment.

$I \equiv(1 / 12) d_{s} t^{3}$ and is the moment of inertia.

$t_{s}$ is thickness of material. in this case the thich.icss of the sting at the point where the strain gauges are mounted (1/16in or $1.59 \mathrm{~mm})$.

$d_{s}$ is width of material. in this case the diameter of the sting $(1,4$ in or $6.35 \mathrm{~mm})$.

$\boldsymbol{\epsilon}$ is strain.

$E$ is Young"s modulus of elasticity for the sting assembly ( $\approx 4960 \mathrm{ksi}$ or $\approx 32.3 \mathrm{GPal}$.

$Y$ is the side force applied on the model, and

$l$ is the distance between the point of application of the side force (centre of press'ire) and the strain gauges.

The following equations relatıng side force and centre of pressure location to measured strain were derned: 


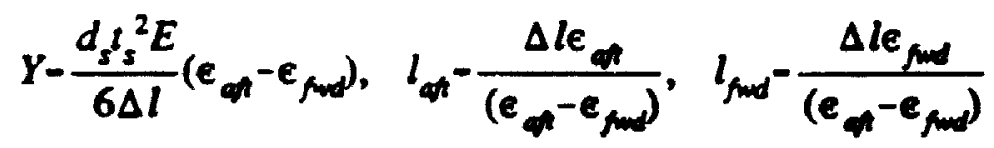

where: $\quad e_{\text {in }}$ is the strain at the aft strain gauge bridge station,

$e_{\text {fud }}$ is the strain at the forward strain gauge bridge station,

$\Delta l$ is the distance between strain gauge bridge stations on the sting $(2.25 \mathrm{in}$ or $5.72 \mathrm{~mm})$,

$l_{a}$ is the distance between the centre of pressure and the aft strain gauge bridge station, and

$l_{\text {fud }}$ is the distance between the centre of pressure and the forward strain gauge bridge station.

These relations were used to calculate the applied side force and to locate its centre of pressure. The resulting yaw moment was then determined as a force-distance product. 
Appendix D: Analysis of Lateral Oscillations for the F-18 Aircraft 


\section{Dimensional Similarity}

To maintain dimensional similarity in nozzle size and position:

$$
\left(\frac{d}{d}\right)_{\text {model }}-\left(\frac{d_{j}}{d}\right)_{F-18} \text { and, }\left(\frac{x}{d}\right)_{\text {model }}-\left(\frac{x}{d}\right)_{F-18}
$$

where: $\quad d_{j}$ is the diameter of the nozzle.

$d$ is the base diameter of the forebody, and

$x$ is the position of the nozzle aft of the apex.

Knowing that $\mathrm{d}_{\text {mastel }}=1$ in $(2.54 \mathrm{~cm})$ and estimating that $\mathrm{d}_{\mathrm{f}-\mathrm{s}}=3 \mathrm{ft}(0.91 \mathrm{~m})$ from figure 4.22 resulted in a full-scale nozzle diameter of 0.720 in $(18.3 \mathrm{~mm})$. On the F-18. these nozzles uould be flush-mounted 9 in $(22.9 \mathrm{~cm})$ aft of the apex and at a radial position of $Q_{1}= \pm 135$ from the windward meridian.

\section{Inertial Response}

The assessment of the inertial response of an F-18 to a fluctuating yau moment input starts with the dynamic equation (valid for pure yawing motion):

$$
N-I_{z i} \dot{r}
$$


where: $\quad N$ is the yaw moment,

$I_{z z}$ is the moment of inertia about the aircraft's $\mathrm{Z}$ axis, a nominally vertical axis through the centre of gravity,

$\dot{r} \equiv \mathrm{dr} / \mathrm{dt}$, is the angular acceleration in yaw.

Note that $r$ is the yau rate and is equivalent to $d \beta^{\prime} d t$. Nou suppose that $N$ varies harmonically in time. then

$$
N-N_{0} \sin (\omega t)
$$

where: $\quad \omega$ is the circular frequency of oscillation in rad/sec. and

$N_{o}$ is the yaw moment amplitude, therefore

$$
\dot{r}=\frac{N_{0}}{l_{z:}} \sin (\omega t)
$$

Integrating twice with respect to time yields:

$$
\beta=-\frac{N_{0}}{I_{z=} \omega^{2}} \sin (\omega t)+C_{1} t+C_{2}
$$

Assuming that all transients have decayed leaving a steady harmonic response, and that $B=0^{\circ}$ at $1=0$. The yaw amplitude can be expressed as: 


$$
\beta_{\max }-\frac{N_{0}}{I_{z} \omega^{2}}
$$

To calculate the moment of inertia, $I_{z}$, of an aircraft about its $Z$ axis, Raymer (1989) provides the following correlation:

$$
l_{z}-\left(\frac{b+L}{2}\right)^{2} \frac{W R_{2}^{2}}{48}
$$

where:

$b$ is the wing span,

$L$ is the length of the aircraft.

$W$ is the weight. and

$\overline{\boldsymbol{R}}_{z}$ is a nondimensional radius of gyration,

$=0.5$ for the F-18 [Rayme:, 1989].

Recalling that the relation between side force and yaw moment coefficients can be expressed as:

$$
C_{n}-C_{\lambda}\left(\frac{x_{c_{p}}}{d}\right)
$$

From figure 4.19(a), at $\alpha=50^{\circ}$ and $\beta=0^{\circ}, C_{y}=3$ and $C_{n}=6$ therefore.

$$
\left(\frac{X_{c_{p}}}{d}\right)_{\text {apex }}-2
$$


With reference to figure 4.22 , estimating a base diameter for the forebody of the F-18 to be approximately $3 \mathrm{ft}(0.91 \mathrm{~m})$ results in the centre of pressure being about $6 \mathrm{ft}(1.83 \mathrm{~m})$ aft of the apex and consequently, about $27 \mathrm{ft}(8.23 \mathrm{~m})$ ahead of the centre of gravity. Thus.

$$
\left(\frac{x_{c e}}{d}\right)_{c G}-9
$$

The resulting yau moment coefficient referenced to the centre of gravity is $C_{r}=27$.

For the comparison made between various yau control methods mentioned earlier. Malcolm (1991) used a free stream velocity of $330 \mathrm{ft} / \mathrm{sec}(100 \mathrm{~m} / \mathrm{sec})$ at an aititude of $15000 \mathrm{ft}(4.6 \mathrm{~km})$. This resulted in a dynamic pressure of $75 \mathrm{psf}(3.59 \mathrm{kPa})$. Applying these values to the present discussion results in a frequency of oscillation of $\mathrm{f}=2.8 \mathrm{~Hz}$ or $\dot{ } \approx 17.6 \mathrm{rad} / \mathrm{sec}$ and a yau moment of $\mathrm{N}_{\mathrm{o}}=42900 \mathrm{ft} \cdot \mathrm{lbs}(58.2 \mathrm{kN} \cdot \mathrm{m})$. Thus the maximum yaw amplitude for an F-18 loaded to a maximum takeoff weight of $50000 \mathrm{lbs}(222 \mathrm{kN})$ is $3_{\operatorname{man}}= \pm 6.54 \times 10^{\circ} \mathrm{rads}\left(0.0375^{\circ}\right)$ and for an empty F-18 weighing 20500lbs (91kN), $B_{\max } \approx \pm 1.59 \times 10^{2}$ rads $\left(0.0913^{\circ}\right)$. These results indicate that the apex of a fully loaded F18 would oscillate about $\pm 0.25 \mathrm{in}(6.35 \mathrm{~mm})$ and so the pilot would experience an oscillatory amplitude of approximately $0.28 \mathrm{in}(7.11 \mathrm{~mm})$ peak to peak. Similarly for an empty F-18, the apex would oscillate roughly \pm 0.63 in $(16.0 \mathrm{~mm})$ while the pilot would $\therefore$ zience an oscillatory amplitude of about 0.68 in $(17.27 \mathrm{~mm})$ peak to peak. 


\section{Appendix E: Water Tunne! Operating Procedures}




\section{Water Tunnel Operating Procedures}

The following is a summary of the procedures outlined in chapter 3 for smooth operation of the water tunnel:

- If the water tunnel has remained idle for a long perind of time (ie: over two months) then, before filling. the supply water should be allowed to pass through the holding tank and into the laboratory sump until the stagnant water in the building supply lines has passed. This results in clearer water at the start.

- Freshly supplied water should sit in the holding tank for about 24 hours before filling the top leg of the water tunnel circuit so that the initial dosage of granular chlorine (about 0.1 ounces or 3 grams) is allowed to fully dissolve. This will also prevent air bubbles from collecting on the plexiglass walls of the test section and on the model.

- While filling the top leg of the water tunnel circuit, air should always be allowed to bleed out of the local high point in the diffuser. downstream of the test section. A quarer-turn stainless steel ball valie has been installed in that area for this purpuse.

- Before experimenting, the water tunnel should run for 10 to 20 minutes to allow the free stream velocity to stabilise.

- The water tunnel should never be left unattended with the test section filled. Overnight. between test runs, water from the test section should be drained into the holding tank. This is the best time to add the 0.1 ounces ( 3 grams) of granular chlorine required every 48 hours to maintain water clarity since the agitation 
caused by the draining operation quickly dissolves the chlorine.

- When shutting down the water tunnel for long penods of time (ie: after experiments have been completed), the drain valves in both the water tunnel and the holding tank should be left open and the access holes at the top of the test section should be left uncovered to allow the water tunnel to completely dry. Also, the dye reservoirs and their respective needle valves should be cleaned and dried to prevent permanent staining of the reservoirs and blockage of the needle valves. 

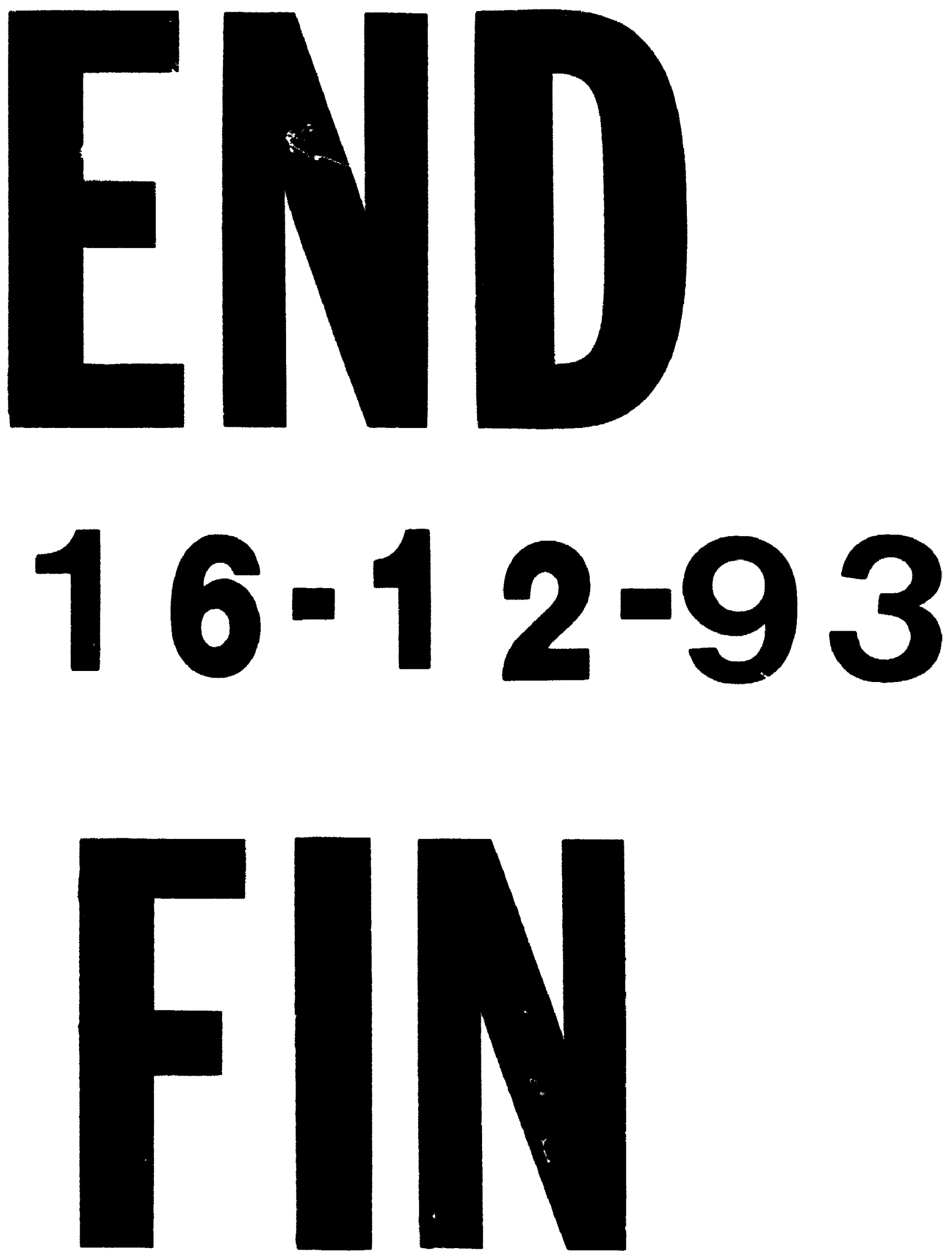